\title{
DEVELOPMENT OF A NONLINEAR FREQUENCY COMPOUNDING METHOD WITH APPLICATIONS IN ULTRASOUND IMAGING AND THERMOMETRY
}

\author{
by \\ Tyler Hornsby \\ B.Sc. University of Calgary, Calgary, Canada, 2017
}

\author{
A thesis \\ presented to Ryerson University \\ in partial fulfillment of the \\ requirements for the degree of \\ Master of Science \\ in the program of \\ Biomedical Physics
}

Toronto, Ontario, Canada 2020

(c) Tyler Hornsby, 2020 


\section{Author's Declaration}

I hereby declare that I am the sole author of this thesis. This is a true copy of the thesis, including any required final revisions, as accepted by my examiners.

I authorize Ryerson University to lend this thesis to other institutions or individuals for the purpose of scholarly research.

I further authorize Ryerson University to reproduce this thesis by photocopying or by other means, in total or in part, at the request of other institutions or individuals for the purpose of scholarly research.

I understand that my thesis may be made electronically available to the public.

Tyler Hornsby 


\title{
Abstract
}

\section{Development of a Nonlinear Frequency Compounding Method With Applications In Ultrasound Imaging and Thermometry}

\author{
Tyler Hornsby \\ Master of Science, Biomedical Physics \\ Ryerson University, 2020
}

Frequency compounding is an ultrasound imaging technique used to reduce artifacts and improve signal-to-noise-ratio (SNR). In this work a new nonlinear frequency compounding (NLFC) method was introduced, and its application in B-mode imaging and noninvasive thermometry was investigated. NLFC input frequencies were optimized to maximize speckle-signal-to-noise-ratio (SSNR) in a tissue mimicking phantom, and the method was then used to produce maps of the temperature sensitive change in backscattered energy of acoustic harmonics $(h \mathrm{CBE})$ during heating of ex vivo porcine tissue with a focused ultrasound transducer. A $h$ CBE-to-temperature calibration was also performed and temperature maps produced. Lastly, a comparative study of the NLFC and previously used nonlinear single frequency (NLSF) method was completed. By using the NLFC method it was concluded that SSNR of B-mode and backscattered energy images, SNR of $h \mathrm{CBE}$ maps, and temperature map agreement with a theoretical COMSOL based model were improved over the previously used NLSF method. 


\section{Acknowledgements}

First and foremost, I wish to express my gratitude to my supervisor Dr. Jahan Tavakkoli for his support and guidance throughout this research. I would also like to extend my gratitude to Dr. Elyas Shaswary for his input in group meetings and help developing and testing the frequency compounding code. Additionally, I would like to thank the members of my supervisory committee, Dr. Carl Kumaradas and Dr. Michael C. Kolios for their time and helpful comments. I would also like to show my appreciation to Dr. Graham Ferrier for his technical help in the experimental design, and guidance with the Comsol simulation.

Finally, I must express my profound gratitude to my parents for providing me with unfailing support and encouragement throughout my years of study and through the process of writing this thesis. This accomplishment would not have been possible without them. Thank you. 


\section{Table of Contents}

Author's Declaration . . . . . . . . . . . . . . . . . . ii

Abstract ........................................

Acknowledgements ............................... iv

List of Tables . . . . . . . . . . . . . . . . . . . viii

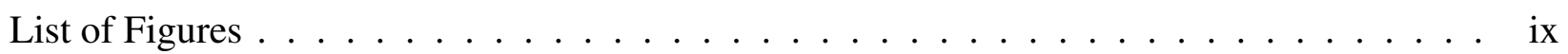

List of Symbols and Abbreviations $\ldots \ldots \ldots \ldots \ldots \ldots \ldots$ xi . . . . . . . . . .

$1 \quad$ Introduction: Ultrasound Imaging and

Noninvasive Thermometry $\ldots \ldots \ldots \ldots \ldots$

$1.1 \quad$ Ultrasound Imaging $\ldots \ldots \ldots \ldots \ldots \ldots$

1.1 .1 Basics of Ultrasound $\ldots \ldots \ldots \ldots \ldots$

1.1 .2 Ultrasound Image Quality $\ldots \ldots \ldots \ldots$

1.2 Thermotherapy and Noninvasive Thermometry $\ldots \ldots \ldots . \ldots 8$

1.2 .1 Thermotherapy Applications and Thermal Dose . . . . . . . . . . . . 8

1.2 .2 Thermotherapy Regimes $\ldots \ldots \ldots$. . . . . . . . . . . . . . . . . 9

1.2 .3 Hyperthermia and Thermal Ablation In Cancer Treatment. . . . . . . . . . 10

1.2 .4 Available Thermotherapy Modalities . . . . . . . . . . . . . . . 11

1.2 .5 Noninvasive Thermometry $\ldots \ldots \ldots \ldots$

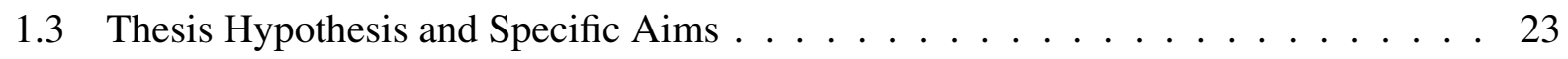

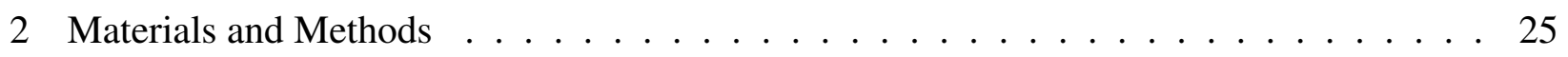


2.1 Development and Optimization of The NLFC Method In Nonlinear B-Mode and BE Imaging . . . . . . . . . . . . . . . . . . . 25

2.1 .1 NLSF Method . . . . . . . . . . . . . . . . . . . . . . . . 26

2.1 .2 NLFC Method . . . . . . . . . . . . . . . . . . . . . 32

2.1 .3 Determining NLFC Input Parameters $\ldots \ldots \ldots \ldots$

2.1.4 Quantifying The Improvement In B-Mode and BE Image SSNR Due To The NLFC Method . . . . . . . . . . . . . . . . . . . . . . 41

2.2 Development and Application of The NLFC Method In Nonlinear Ultrasound Thermometry . . . . . . . . . . . . . . . . . . . . . . 41

2.2 .1 NLSF Method $h$ CBE Calculation . . . . . . . . . . . . . . . . 42

2.2 .2 NLFC Method $h$ CBE Calculation . . . . . . . . . . . . 42

2.2 .3 LIFU Heating of Ex Vivo Porcine Muscle Tissue. . . . . . . . . . . . . . 44

$2.2 .4 \quad h$ CBE-to-Temperature Calibration $\ldots \ldots \ldots \ldots \ldots$

2.2 .5 COMSOL Simulation of 2D Temperature Maps . . . . . . . . . . . . 46

2.2 .6 Quantifying SNR Improvement With The NLFC Method . . . . . . . . . . 48

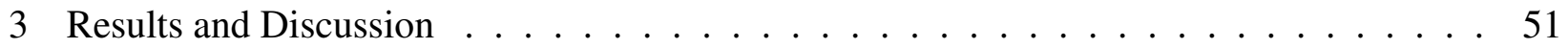

$3.1 \quad$ Development and Optimization of The NLFC Method In Nonlinear B-Mode and BE Imaging . . . . . . . . . . . . . . . . . . . 51

3.1 .1 Determining NLFC Input Frequencies $\ldots \ldots \ldots \ldots \ldots$

3.1 .2 Determining Number of Cycles of Excitation Pulse . . . . . . . . . . 54

3.1.3 A Comparative Study In B-Mode and BE Image SSNR: NLFC vs NLSF

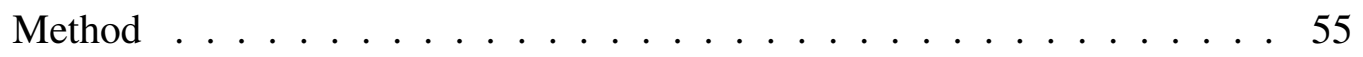

3.2 Development and Application of The NLFC Method In Nonlinear Ultrasound Thermometry . . . . . . . . . . . . . . . . . . 57

$3.2 .1 \quad$ LIFU Heating 2D $h$ CBE Maps $\ldots \ldots \ldots \ldots \ldots$

$3.2 .2 \quad h$ CBE-to-Temperature Calibration $\ldots \ldots \ldots \ldots$

$3.2 .3 \quad$ A Comparative Study: The NLFC vs NLSF Method . . . . . . . . . . . . 63

4 Summary, Conclusions and Future Works $\ldots \ldots \ldots \ldots$. . . . . . . . . . 68

4.1 Summary of Work $\ldots \ldots \ldots \ldots$ 
4.2 Conclusions $\ldots \ldots \ldots \ldots$

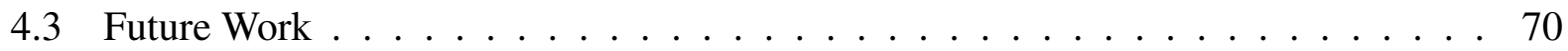

Bibliography . . . . . . . . . . . . . . . . . . . . 72 


\section{List of Tables}

2.1 Important thermal and acoustic parameters used in the COMSOL simulation . . . . 48

3.1 Improvements in B-mode SSNR due to the NLFC method . . . . . . . . . . . 56

3.2 Improvements in backscattered energy SSNR due to the NLFC method . . . . . . . 56

3.3 SNR of $B E_{N L F C}$ and $B E_{\text {single }} h \mathrm{CBE}$ maps for LIFU heating of ex vivo porcine tissue 65

3.4 Improvements in $h \mathrm{CBE}$ map SNR due to the NLFC method . . . . . . . . . . 65

3.5 NLFC vs NLSF methods RMSE $\ldots \ldots \ldots$. . . . . . . . . . . . . 67 


\section{List of Figures}

$1.1 \quad$ B-mode image generation with a typical linear array transducer . . . . . . . . . 2

$1.2 \quad$ A comparison of frequency compounded and conventional B-mode images . . . . . 5

1.3 Production of acoustic harmonics during nonlinear wave propagation . . . . . . . . 6

1.4 Improvements in B-mode image quality from ultrasound harmonic imaging . . . . 7

1.5 The use of hyperthermia in active targeting of tumours with chemotherapeutic drugs 11

2.1 L12-5 (50mm) linear array transducer frequency response curve $\ldots . . . . . .26$

2.2 Filtering the fundamental and second harmonic signals . . . . . . . . . . 27

2.3 The generation of an $S(x, y)$ frame from 256 time signals $\ldots \ldots$. . . . . . . . 28

2.4 A step-by-step diagram of the NLSF method used in the generation of B-mode images 30

2.5 A detailed block diagram outlining the generation of B-mode and BE images using the NLSF method $\ldots \ldots \ldots \ldots$. . . . . . . . . . . . . . . 32

2.6 A step-by-step diagram of the NLFC method used in the generation of B-mode images 35

2.7 A detailed block diagram outlining the generation of B-mode and BE images using the NLFC method . . . . . . . . . . . . . . . . . . . . . . . 37

2.8 Tissue mimicking phantom B-mode imaging experimental setup . . . . . . . . 38

2.9 Determining B-mode Image SSNR . . . . . . . . . . . . . . . . . . . 39

2.10 A comparison of the NLSF and NLFC methods . . . . . . . . . . . . 43

2.11 Experimental setup for LIFU heating of ex vivo porcine muscle tissue . . . . . . 45

2.12 The 3D multi-layer geometry simulated using COMSOL . . . . . . . . . . . . 47

2.13 Determining SNR of 2D $h$ CBE maps for LIFU heating . . . . . . . . . . . . . 49

2.14 Lateral line profile calculation method for experimental 2D temperature maps . . . 50

3.1 Transmitted frequency dependence of $B_{\text {Single }}$ SSNR $\ldots \ldots \ldots$. . . . . . . . 52 
$3.2 \quad B_{N L F C}$ SSNR for all possible frequency pairs in the $B_{\text {Single }}$ SSNR peak . . . . . 52

3.3 Transmitted frequency dependence of $B E_{\text {Single }}$ SSNR $\ldots \ldots \ldots$

$3.4 B E_{N L F C}$ SSNR for all possible frequency pairs in the $B E_{\text {Single }}$ SSNR peak . . . . 53

$3.5 \quad$ SSNR dependence on the number of cycles for various frequency combinations . . 54

3.6 COMSOL simulation of LIFU transducer intensity field . . . . . . . . . . . . 55

3.7 Comparing $B_{N L F C}$ and $B_{\text {Single }}$ images $\ldots \ldots \ldots \ldots$. . . . . . . . 57

3.8 A typical $h$ CBE map obtained using the NLFC method . . . . . . . . . . . 58

$3.9 \quad$ A typical $h$ CBE map obtained using the NLSF method with a $4.17 \mathrm{MHz}$ transmit

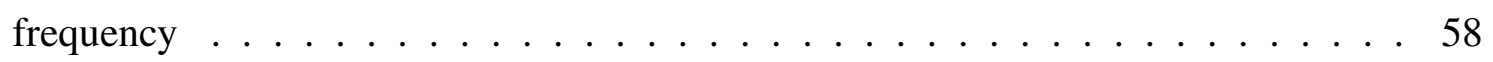

3.10 A typical $h$ CBE map obtained using the NLSF method with a $5.43 \mathrm{MHz}$ transmit frequency . . . . . . . . . . . . . . . . 59

3.11 Reduction of motion artifacts using the motion compensation function and gradient filter . . . . . . . . . . . . . . . . . . 60

3.12 Temperature vs $h \mathrm{CBE}$ focal point measurements for LIFU heating of ex vivo porcine

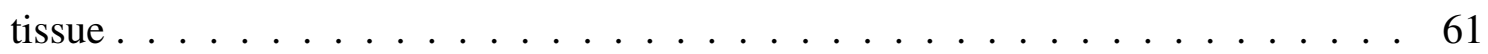

$3.13 h$ CBE-to-temperature calibration curve . . . . . . . . . . . . 62

3.14 A typical 2D temperature map obtained using the NLFC method . . . . . . . . 62

3.15 A typical 2D temperature map obtained using the NLSF method with a $4.17 \mathrm{MHz}$ transmit frequency . . . . . . . . . . . . . . . . . . 63

3.16 A typical 2D temperature map obtained using the NLSF method with a $5.43 \mathrm{MHz}$ transmit frequency . . . . . . . . . . . . . . . . . 63

3.17 Measured SNR of 2D $h$ CBE maps generated using both the NLFC and NLSF methods 64

3.18 A simulated 2D temperature map obtained using COMSOL . . . . . . . . . . . 66

3.19 RMSE as a function of time for NLFC and NLSF methods . . . . . . . . . . 66 


\title{
List of Symbols and Abbreviations
}

\author{
Chapter 1 \\ NLFC Nonlinear Frequency Compounding \\ NLSF $\quad$ Nonlinear $\underline{\text { Single Frequency }}$ \\ SNR $\quad$ Signal-to-Noise Ratio \\ SSNR $\quad$ Speckle- $\underline{\text { Signal-to-Noise Ratio }}$ \\ MRI $\quad$ Magnetic Resonance Imaging \\ THI Tissue $\underline{\text { Harmonic Imaging }}$ \\ SOS $\quad$ Speed $\underline{\text { Of }} \underline{\text { Sound }}$ \\ $h_{1} \quad$ Fundamental frequency signal \\ $h_{2} \quad$ Second harmonic signal \\ $h_{3} \quad$ Third harmonic signal \\ DTHI Differential Tissue Harmonic Imaging \\ $f_{1} \quad$ Center frequency 1 (lower frequency) \\ $f_{2} \quad$ Center frequency 2 (higher frequency) \\ SHI $\quad$ Super-Harmonic Imaging \\ ROI $\quad$ Region $\underline{\text { Of Interest }}$ \\ CEM43 Equivalent minutes thermal dose estimation \\ t Time \\ $t_{43} \quad$ Cumulative equivalent minutes at $43^{\circ} \mathrm{C}$ \\ $t_{\text {final }} \quad$ Total exposure time during thermotherapy \\ $\bar{T} \quad$ Average temperature during heating time interval \\ HT $\quad$ Hyperthermia Treatment
}




\begin{tabular}{|c|c|}
\hline TSL & Thermo-Sensitive Liposome \\
\hline GNP & $\underline{\text { Gold } \underline{\text { Nano- }} \text { Particle }}$ \\
\hline $\mathrm{RF}$ & $\underline{\text { Radio-Erequency }}$ \\
\hline LITT & $\underline{\text { Laser }} \underline{\text { Interstitial }} \underline{\mathrm{Th}}$ ermal $\underline{\text { Therapy }}$ \\
\hline$\mu_{a}$ & Absorption coefficient \\
\hline HIFU & $\underline{\text { High }} \underline{\text { Intensity }} \underline{\text { Focused }} \underline{\text { Ultrasound }}$ \\
\hline LIFU & Low Intensity Focused $\underline{\text { Ultrasound }}$ \\
\hline MR & $\underline{\text { Magnetic Resonance }}$ \\
\hline$T_{1}$ & Spin-lattice relaxation time \\
\hline$T_{2}$ & Spin-spin relaxation time \\
\hline PRF & Proton $\underline{R}$ esonance Frequency \\
\hline$\omega$ & Resonance frequency \\
\hline$\gamma$ & Gyromagnetic ratio \\
\hline$B_{\text {local }}$ & Local magnetic field \\
\hline$s$ & Screening constant \\
\hline$B_{0}$ & Magnetic flux density \\
\hline MRgLITT & 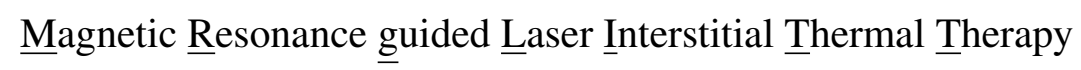 \\
\hline MRgFUS & 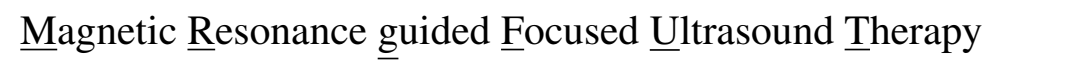 \\
\hline$B E$ & Backscattered Energy \\
\hline$I(x)$ & Average signal intensity at some depth $x$ \\
\hline$I(0)$ & Average signal intensity at depth $x=0$ \\
\hline$\alpha$ & Attenuation coefficient \\
\hline$f$ & Center frequency of transmitted ultrasound wave \\
\hline$\alpha_{0}$ & Attenuation coefficient at $1 \mathrm{MHz}$ \\
\hline$n$ & Frequency dependant constant \\
\hline $\mathrm{CBE}$ & Change in Backscattered Energy \\
\hline$h \mathrm{CBE}$ & 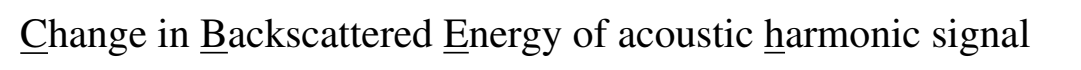 \\
\hline$\tau$ & Time \\
\hline$\kappa$ & Adiabatic compressibility \\
\hline$c$ & Ultrasound wave velocity \\
\hline
\end{tabular}




$\begin{array}{ll}\rho_{0} & \text { Medium density at equilibrium } \\ z & \text { Depth } \\ \delta T(z) & \text { Temperature change at some depth } z \\ c_{0} & \text { Measured sound velocity at a set temperature and pressure } \\ \alpha_{L} & \text { Linear coefficient of thermal expansion of medium } \\ \lambda & \text { Thermal coefficient of the SOS } \\ \delta t(z) & \text { Cumulative time shift at some depth } z \\ B / A & \text { Parameter of nonlinearity } \\ s & \text { Entropy } \\ P & \text { Pressure } \\ \rho & \text { Medium density } \\ T & \text { Temperature } \\ \alpha_{p} & \text { Isobaric thermal expansion coefficient } \\ c_{p} & \text { Isobaric specific heat capacity } \\ v_{\theta} & \text { Phase Velocity } \\ v & \text { Particle velocity } \\ \beta & \text { Coefficient of nonlinearity }\end{array}$

\section{Chapter 2}

$\begin{array}{ll}\text { TMP } & \text { Tissue } \underline{\text { Mimicking }} \text { Phantom } \\ p & \text { Transmitted pulse } \\ s & \text { Received signal in time domain } \\ t & \text { Time } \\ S & \text { Received signal in frequency domain } \\ \omega & \text { Frequency } \\ \mathcal{F} & \text { Fourier transform } \\ \mathcal{F}^{-1} & \text { Inverse Fourier transform } \\ g & \text { Gaussian filter } \\ S(x, y) & \text { Frame of time signals } \\ \mathcal{H} & \text { Hilbert transform } \\ L & \text { Logarithmic compression function }\end{array}$


B-mode image

$D$

Stabilizing constant

$\operatorname{Max}$

Maximum pixel value in frame

$\bar{B}$

Average B-mode image

$f_{1}$

Frequency 1

$f_{2}$

Frequency 2

$\mu_{S}$

Mean signal intensity in uniform region

$\sigma_{S}$

Standard deviation of signal in uniform region

$S P L$

Spatial pulse length

FWHM $\quad$ Full Width $\underline{\text { Half }}$ Maximum

$A R L$

Axial resolution limit

$\lambda$

Wavelength of transmitted wave

$N$

$f$

Number of Cycles

Transmitted frequency

$c$

Speed of sound in tissue mimicking phantom

$B E$

Backscattered energy

$\tau$

LIFU heating time stamp

$\overline{B E}$

Average backscattered energy

$h$ CBE' Harmonic CBE between each consecutive time stamp

RMSE $\quad \underline{\text { Root }} \underline{\text { Mean }} \underline{\text { Squared Error }}$

$\alpha_{\text {Tissue }}$

Attenuation Coefficient of Porcine Tissue

$\alpha_{W}$

Attenuation Coefficient of Water

$C_{P, \text { Tissue }}$

Heat Capacity at Constant Pressure of Porcine Tissue

$C_{P, W}$

Heat Capacity at Constant Pressure of Water

$\rho_{\text {Tissue }}$

Density of Porcine Tissue

$\rho_{W}$

Density of Water

$k_{\text {Tissue }}$

Thermal Conductivity of Porcine Tissue

$k_{W}$

Thermal Conductivity of Water

$c_{\text {Tissue }}$

Speed of Sound in Porcine Tissue

$c_{W}$

Speed of Sound in Water 


\section{$1 \mid$ Introduction: Ultrasound Imaging and Noninvasive Thermometry}

In this chapter a comprehensive literature review is provided covering the two main contributions of this work. Namely: (1) The development of a nonlinear frequency compounding (NLFC) method and optimization of input parameters based on nonlinear ultrasound B-mode imaging in a tissue mimicking phantom. (2) The development and application of the NLFC method in nonlinear ultrasound thermometry in ex vivo tissue. In section 1.1 the basics of ultrasound B-mode imaging and artifact reduction techniques are explored. In section 1.2 the topics of thermotherapy and noninvasive thermometry are covered. Lastly, in section 1.3 the thesis hypothesis and specific aims are provided.

\subsection{Ultrasound Imaging}

\subsubsection{Basics of Ultrasound}

The term "ultrasound" refers to all acoustic energy with a frequency above the range of audible sound $(20 \mathrm{~Hz}-20 \mathrm{kHz})$ [1]. Due to its ability to obtain both anatomical and functional images of the human body, ultrasound has seen extensive use as an imaging modality in clinical applications. Additionally, its non-ionizing, non-invasive nature, and inexpensive cost of operation can prove advantageous when compared to other clinical imaging modalities such as conventional X-ray or magnetic resonance imaging (MRI) [2].

In clinical applications ultrasound imaging is performed primarily using a pulse-echo method 
with brightness mode (B-mode) display, where a brightness value is assigned corresponding to echo intensity. In this technique an ultrasound transducer is excited by an electrical pulse and a subsequent transmitted ultrasound pulse is generated in the form of an acoustic wave. The generated acoustic wave will propagate through the body, with tissues along the path of transmission of various acoustic impedance causing a fraction of the transmitted wave to be reflected back to the transducer as an echo [2, 3]. Echos are then classified based on both the amplitude and time it takes to receive the echo, and a 2D image is formed by combining a large number of echo signals as a B-mode line. In the generated 2D B-mode image the horizontal location of pixels is determined by the location at which the echo returns along the the probe face, while vertical pixel placement is determined by the total travel time of the ultrasound wave. A brightness value is then applied to each pixel corresponding to the echo amplitude. As an example, fluid transmits more ultrasound waves than solid material, leading to a lower level of reflection. This produces an echogenic black image, while a dense material like bone causes high reflection and produces white bright pixels [4, 5]. A simplified example of B-mode image generation with a typical linear array transducer is provided in Figure 1.1.
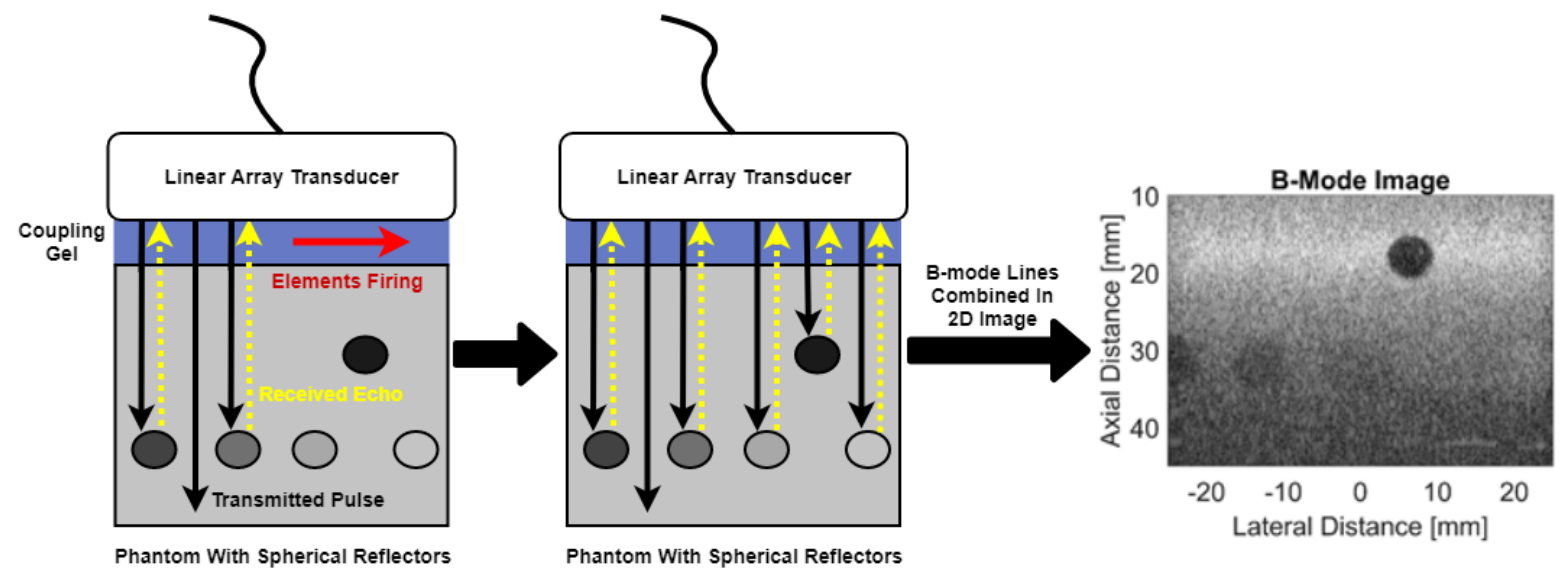

Figure 1.1: A typical linear array transducer is used to image a phantom with spherical reflectors. Transmitted pulses are shown as solid black arrows, while reflected echos are shown as dashed yellow arrows. (Left) All linear array transducer elements are fired sequentially with the direction of firing shown in red. (Middle) All elements have been fired and echos are received from all spherical reflectors in the phantom. These echos are then assigned a brightness value corresponding to signal strength and a (lateral, axial) coordinate based on the time between transmission and receiving to generate B-mode lines. (Right) All B-mode lines are then combined to form a 2D image of the phantom. Note that a coupling gel (shown in blue) was included between the phantom and linear array transducer to avoid reflection at the air-phantom boundary due to large differences in acoustic impedance. 
Ultrasound transducers contain multiple piezoelectric crystals, which through the piezoelectric effect can convert electric energy into mechanical energy and vice versa [3]. In order to produce an acoustic wave the piezoelectric material of a transducer is excited with an electrical signal. If the transmitted wave then strikes a spherical reflector in the body a portion of the reflected waves will be backscattered to the transducer. This returning wave will then be converted back into an electrical signal, amplified, and recorded as a voltage versus time pulse by the transducer [2, 3].

Typical diagnostic ultrasound scanners operate in the frequency range of 2 to $18 \mathrm{MHz}$, with a variety of sizes and geometries available for specific applications [1]. Commonly used transducer geometries include single element and linear arrays. Single element transducers are usually operated at high frequencies and can form 2D images through mechanical translation or rotation of a single element. In linear array transducers electronic scanning can be utilized to improve temporal resolution, and as a result they are more commonly used clinically. The electronic scanning technique mimics single element mechanical motion by firing elements sequentially to effectively move the ultrasound beam [1]. In imaging applications the transducer frequency is essential for providing optimal spatial resolution. High frequency ultrasound waves generate images of high axial resolution, however due to frequency dependant attenuation the penetration depth of high frequency scanners is lower. Conversely, low frequency ultrasound waves offer a lower axial resolution but can image anatomical structures at greater depths due to lowered attenuation [3].

\subsubsection{Ultrasound Image Quality}

\section{Ultrasound Artifacts}

Conventional ultrasound images can be affected by a number of artifacts, with two prominent examples including speckle and clutter. Speckle is a result of the large number of sub-wavelength scatterers within the human body, which can cause constructive and destructive interference in ultrasound waves returning to the transducer. As a result, images produced with an ultrasonic imaging system are inherently plagued with granular noise. In imaging applications speckle can limit the detection of low contrast or small objects, and blur boundaries between different tissue types [6, 7, 8, 9]. 
Clutter is a ubiquitous phenomenon that appears as diffuse echoes overlying structures of interest in ultrasound imaging [10]. It is composed of spurious signals arising from echoes produced outside the main ultrasound beam. Sources of clutter include side lobes, grating lobes, and reverberation [11]. Additionally, noise artifacts can appear due to electronic or image reconstruction artifacts. Improvements in image quality can be achieved through reducing these artifacts; either through improvements in transducer design, or advanced signal acquisition and processing methods. To this end compounding and harmonic imaging have been shown to reduce speckle and clutter in ultrasound imaging [12].

\section{Compounding Techniques In Ultrasound Imaging}

Compounding is an imaging technique which combines frames acquired from either multiple angles and aperture positions (spatial compounding), or frames acquired using multiple frequencies (frequency compounding), to reduce artifacts and improve resolution and SNR without sacrificing potentially important image information [12, 13]. If $N$ images are used in the compounding process, then the typical reduction in speckle is of the order $\sqrt{N}$ for statistically independent images $[12$, 13, 14]. Here, the amount of speckle is quantified by a speckle contrast value equal to the ratio of standard deviation over mean intensity of pixel values in a uniform region [15].

In spatial compounding several frames can be obtained through steering the transducer to predetermined angles, or through physical translation of the transducer. These frames can then be aligned using cross-correlation and averaged to produce a compounded image with improved contrast resolution and SNR [12]. Additionally, spatially compounded images contain lower levels of acoustic artifacts as scanning from different angles produces uncorrelated artifact patterns, which can be averaged out to improve image quality [13]. One drawback of spatial compounding is the reduction in frame rate due to the need for multiple images. To this end, a solution was proposed by Behar et al. in the form of a three transducer system. Here a central transducer is used as both the transmitter and receiver with two lateral unfocused transducers that perform only as receivers, allowing compounding to be performed with no drop in frame rate [16].

Frequency compounding can be performed by acquiring multiple images at separate transmit frequencies, or sub-dividing the frequency of the received spectrum into separate images with or 
without weighting factors [2, 12]. These images can then be averaged to reduce artifacts and improve SNR. Since the appearance of speckle pattern is dependant on the frequency of the ultrasound waves, images obtained using different frequencies have uncorrelated or partially correlated speckle patterns [2]. It has been shown that by using frequency compounding, image SNR and contrast resolution can be enhanced [2, 12, 17]. Due to the improvements in SNR and contrast resolution, frequency compounding can prove advantageous for tissue differentiation while imaging the breast, peripheral blood vessels, or musculoskeletal injuries [13]. An example of frequency compounding improvements in image quality is provided in Figure 1.2 for a breast lesion surrounded by fatty tissue. When implementing frequency compounding, boundaries between the lesion and background tissue are easier to differentiate [18].
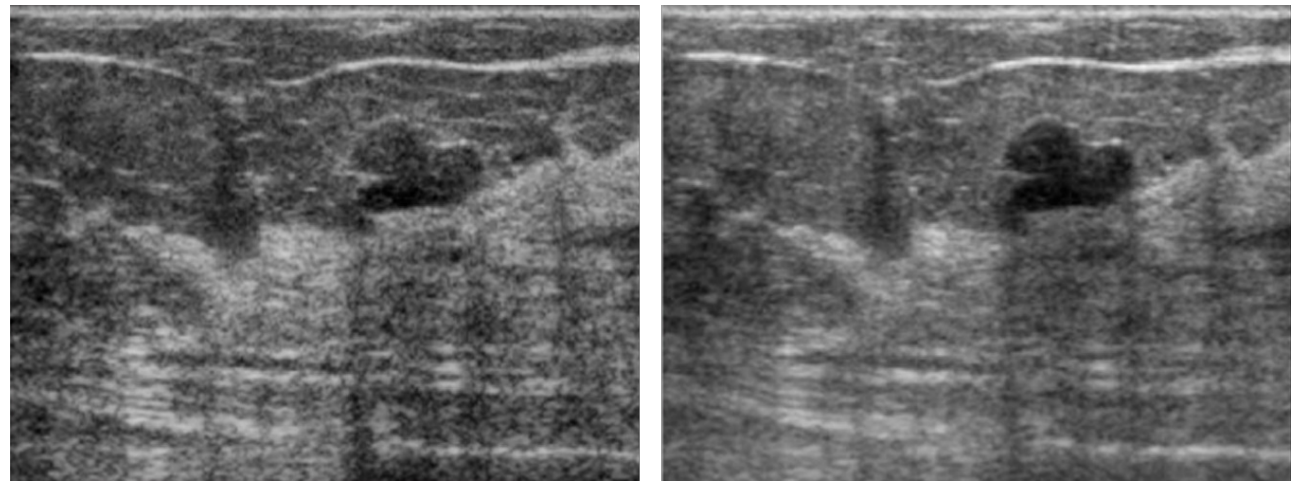

Figure 1.2: In vivo B-mode images of a breast lesion surrounded by fatty tissue, obtained with a linear array transducer. (Left) A conventional B-mode image obtained with a center frequency of 14 MHz. (Right) A frequency compounded B-mode image with center frequencies of $10 \mathrm{MHz}$ and 14 MHz. (Figure adapted from Mesurolle et al. [18])

One limitation of frequency compounding is the fixed bandwidth of many clinical ultrasound imaging systems [12]. To address this Sanchez et al. have proposed a coded excitation and pulse compression technique known as resolution enhancement compression, which selectively excites an ultrasonic source with different energies at predetermined frequencies. Using this technique combined with frequency compounding, the usable bandwidth and axial resolution was effectively doubled [19]. In another approach by Chang et al. a concentric annular high frequency dual element transducer was developed to broaden usable bandwidth. The transducer elements operated at 20 and $40 \mathrm{MHz}$, with the results showing image SNR could be increased with only a small reduction in axial resolution [20]. 


\section{Ultrasound Harmonic Imaging}

One imaging technique reported to reduce clutter is that of tissue harmonic imaging (THI). In this technique, higher order harmonics generated during nonlinear wave propagation are imaged. During propagation of ultrasound waves through a medium the wave form can be modified both by the nonlinearity of the medium and frequency dependant attenuation [21]. Distortion in the shape of a waveform due to nonlinear propagation is a result of variations in the speed of sound (SOS) at different phases of the wave [22]. It is generally assumed that the SOS for small-amplitude waves is constant for all phases of the waves, however this assumption does not hold for high pressure or finite-amplitude waves. In these cases the SOS during the compression phase is increased, while SOS during the rarefaction phase is decreased. This change in SOS is due to the inelastic nature of tissue, meaning that tissue contracts less than it expands. As tissue density is changed in both the compression and rarefaction phase there is a corresponding change in SOS. During tissue contraction in the compression phase, tissue density increases and causes a slight increase in SOS. As the wave returns to the rarefraction phase, tissue expands and the SOS is decreased. This process is known as nonlinear wave propagation, and leads to the progressive distortion of the wave as it propagates. This in turn causes the generation of secondary higher frequency acoustic harmonics signals, known as acoustic harmonics [23]. A visual representation of acoustic harmonic generation is provided in Figure 1.3.
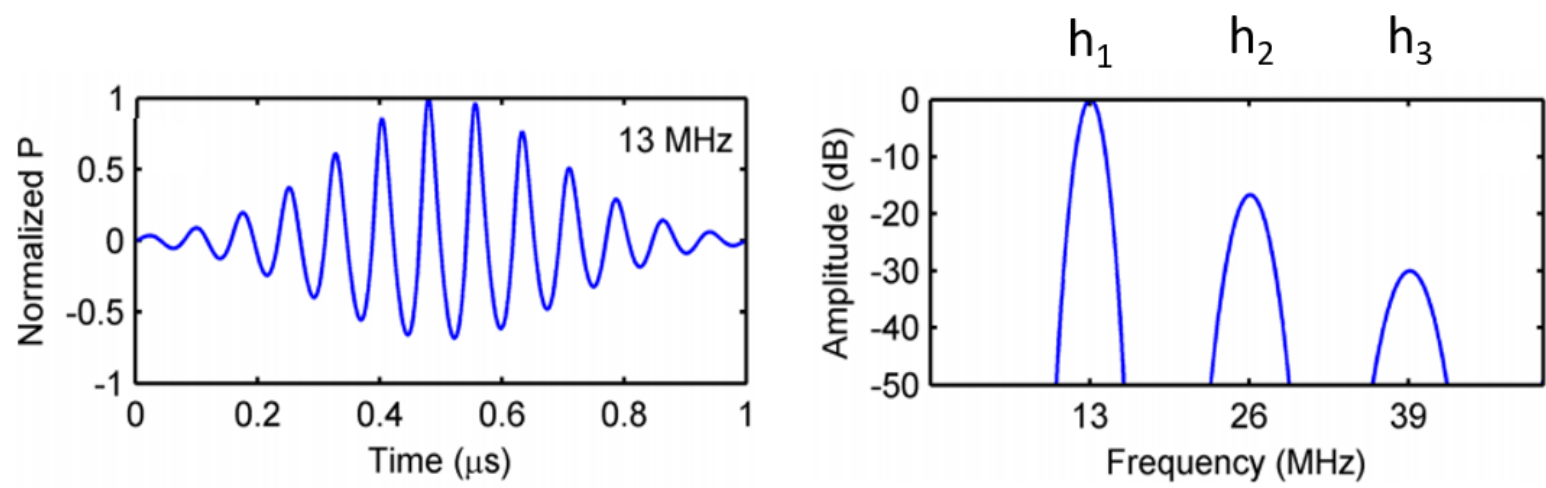

Figure 1.3: Simulated time (left) and frequency (right) spectra for a $13 \mathrm{MHz}$ signal after travelling 3 $\mathrm{cm}$ in water. Here the fundamental signal is visible as a peak at $13 \mathrm{MHz}$ in the frequency spectrum. The second and third acoustic harmonic signal are visible at $26 \mathrm{MHz}$ and $39 \mathrm{MHz}$ respectively, appearing as a result of nonlinear wave propagation [24]. Here $h_{1}$ denotes the fundamental frequency component of the signal, while the second and third harmonics are labelled as $h_{2}$ and $h_{3}$ respectively. 
As the wave continues to propagate, a fraction of the energy from the fundamental frequency $\left(h_{1}\right)$ is transferred to the harmonic frequencies at integer multiples of the fundamental frequency. This nonlinear effect is cumulative with harmonic amplitudes increasing to a maximum depth near the focal point for focused transducers. At any depth past this maximum, frequency dependant attenuation will attenuate the higher harmonics and dominate over nonlinear effects, reducing harmonic amplitude. This nonlinear effect is stronger at higher frequencies and source pressure [10, 22, 24].
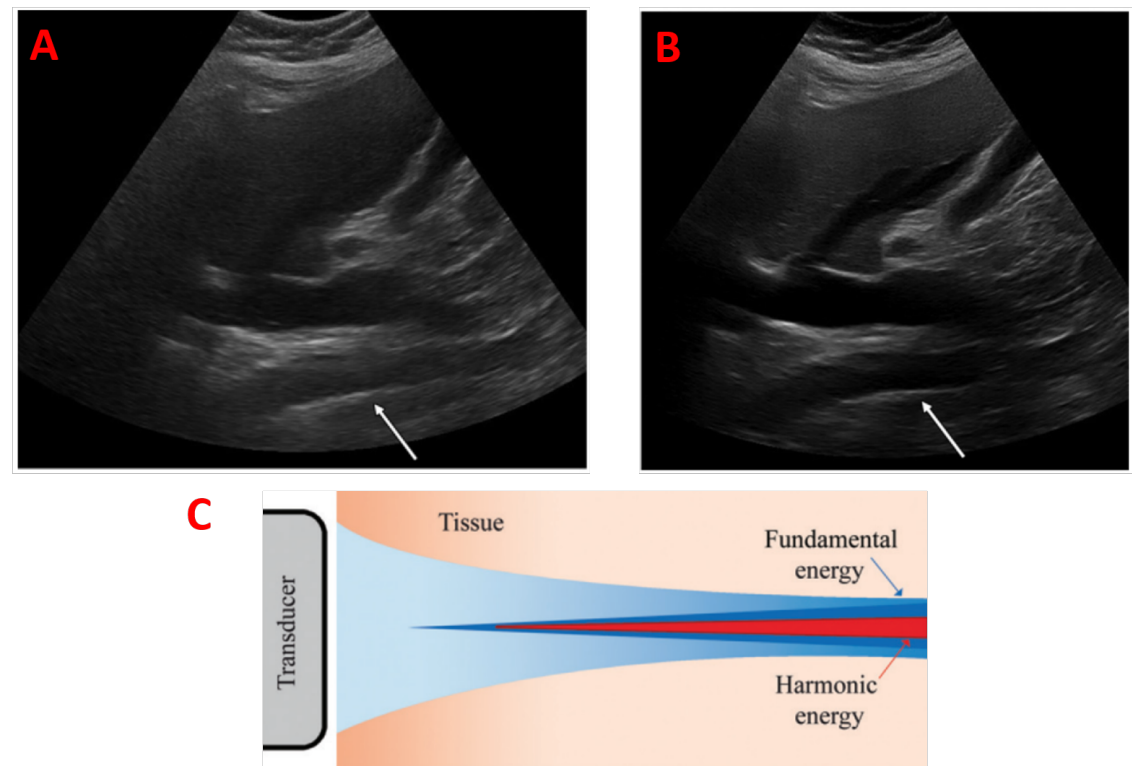

Figure 1.4: (A) A standard B-mode image of the upper abdomen. (B) A B-mode image of the same area acquired using DTHI. Here there are clear improvements in visualization of tissue boundaries, with a significant reduction in speckle at the aorta (white arrow). (C) Energy profile of the fundamental (blue) and harmonic (red) signals. Since harmonics are produced predominantly in the central part of the transmitted beam there is a narrower imaging plane and reduced side and grating lobe artifacts. (Figure adapted from Anvari et al. [23]).

In conventional B-mode images side and grating lobes appear as low amplitude ultrasound beams projecting radially from the main transmitted beam, and can create additional echoes in the final image. Because nonlinear effects are dominant in greater tissue depths and not near the transducer, side and grating lobes can be suppressed in harmonic images. An illustration of this side and grating lobe suppression is provided in Figure 1.4.C. Additionally, THI has been shown to improve axial, lateral, and contrast resolution in B-mode images when compared to conventional 
B-mode imaging techniques [22]. In more recent work, the advantages of THI are beginning to become fully realized with the development of commercial THI techniques such as differential tissue harmonic imaging (DTHI). In this technique a dual frequency pulse is transmitted centered at two distinct frequencies $f_{1}$ and $f_{2}$. The received signal contains echoes at the sum $\left(f_{1}+f_{2}\right)$ and difference $\left(f_{1}-f_{2}\right)$ of the two frequencies, as well as their second harmonics. Only the lower frequency components of the differential frequencies and second harmonic of the lower transmitted frequency will fall inside a clinical transducers bandwidth. using these two received components an improved DTHI B-mode image can be produced [23, 25]. A comparison of a B-mode image obtained using conventional and DTHI techniques is provided in Figure 1.4.A and 1.4.B.

In more recent work the advantages of harmonic imaging have also been combined with frequency compounding techniques, in a technique coined as superharmonic imaging (SHI). In this technique the third and fifth harmonic signals which arise during nonlinear wave propagation were compounded for two different transmitted frequencies. In this system a low frequency subarray, used for transmission, is combined with a high frequency subarray, used for reception, in a specialized phased array transducer to meet bandwidth requirements. Using transmit frequencies of 0.86 and 1.0 $\mathrm{MHz}$, in vitro images were obtained of mitral valve leaflets, clearly showing the dynamics of the systolic phases [26, 27].

\subsection{Thermotherapy and Noninvasive Thermometry}

\subsubsection{Thermotherapy Applications and Thermal Dose}

In thermotherapy the transfer of thermal energy is utilized in order to supply heat to a region of interest (ROI) for therapeutic purposes. The use of heat in medicine is not a new treatment, and has been well documented for centuries. As stated by Hippocrates (479-377 B.C.): “Those who cannot be cured by medicine can be cured by surgery. Those who cannot be cured by surgery can be cured by fire. Those who cannot be cured by fire, they are indeed incurable" [28]. Even at this time the potential of heat to shrink or treat tumours had been observed, and since the time of Hippocrates, the fundamental effects of heat on cancer cells has become well understood. Currently, there are a number of documented clinically significant effects due to the application of heat to a 
ROI. These effects include: the destruction of malignant cells via the induction of necrosis and apoptosis, increased susceptibility of cancer cells to chemotherapeutic drugs, and the activation of immunomodulating effects such as increased perfusion and blood flow to the tumour tissue [28, 29 , 30].

In order to quantify the biological effect of thermotherapy on a patient, a "thermal dose" can be utilized as a function of both temperature and treatment time. Cell death within a ROI from exposure to heat is a function of both the intensity of the applied heat and the length of the exposure or treatment [28]. When quantifying thermal dose, a normalized parameter is used to convert any exposure time and temperature to an equivalent exposure time in minutes at a $43^{\circ} \mathrm{C}$ reference temperature. This estimation, known as CEM43, allows a variety of different thermotherapy treatments to be compared in terms of their thermal dose and prognostic abilities [31]. Currently CEM43 is recognized as the most common form of thermal dosimetry [32].

For a sufficiently small time period between temperature measurements $(\Delta t)$ during tissue heating the equivalent time at a $43^{\circ} \mathrm{C}$ reference temperature $\left(t_{43}\right)$ can be calculated using Equation 1.1 as follows:

$$
t_{43}=\sum_{t=0}^{t=t_{\text {final }}} R^{(43-\overline{T(t))}} \Delta t \quad, \quad R= \begin{cases}0.50 & T>43^{\circ} \mathrm{C} \\ 0.25 & T<43^{\circ} \mathrm{C}\end{cases}
$$

Here $\overline{T(t)}$ is the average temperature during the time interval $\Delta t$, and $t_{\text {final }}$ is the total exposure time during treatment [31]. Using this relationship both the exposure time and temperature can be manipulated to achieve treatment goals.

\subsubsection{Thermotherapy Regimes}

Thermotherapy is a generalized form of therapy, and can be used in a large variety of medical applications. Because of this, thermotherapy treatment can be classified into one of three main treatment types based on temperature and treatment time. These can be differentiated by treatment temperature and are known as diathermia, hyperthermia, and thermal ablation. Diathermia involves the heating of an ROI with relatively low temperatures up to $41^{\circ} \mathrm{C}$ for use in physiotherapy and the 
treatment of rheumatic diseases.

Hyperthermia treatment (HT) can be used to target either a part of the body or in whole body treatment, and operates in the temperature range of $41^{\circ} \mathrm{C}-45^{\circ} \mathrm{C}$. The primary goal of $\mathrm{HT}$ is not to directly produce cell death in a target, but to bolster the effectiveness of standard treatment methods such as chemotherapy and radiation. As of now, HT has been successfully used as a sensitizer in combination with chemotherapy and radiation therapy in solid tumours [32].

Lastly, thermal ablation involves the use of high temperatures above $45^{\circ} \mathrm{C}$ to achieve localized cell death in a target. At temperatures $>55^{\circ} \mathrm{C}$ coagulative necrosis can be induced, and used to ablate a ROI [32]. Like HT, thermal ablation can prove beneficial in oncology. Out of these three main thermotherapy regimes, only HT and thermal ablation are clinically approved for cancer treatment [29, 33, 34].

\subsubsection{Hyperthermia and Thermal Ablation In Cancer Treatment}

Due to their hypoxic nature and insufficient blood perfusion many tumours possess radioresistant properties, meaning treatment with ionizing radiation or chemotherapy can be difficult. As stated by the oxygen fixation hypothesis, these hypoxic environments do not allow the oxidation of DNA free radicals by oxygen and the subsequent "fixing" of radiation induced DNA breaks [32, 35]. In these cases there is strong motivation for the use of HT, as it has been shown to increase blood flow, oxygenation and perfusion, and spacing between endothelial junctions in a targeted region. Additionally, temperatures between $40^{\circ} \mathrm{C}-44^{\circ} \mathrm{C}$ have been shown to be cytotoxic for cells in the low $\mathrm{pO}_{2}$ and $\mathrm{pH}$ environment which exist in tumour environments [36]. Overall, the use of HT can increase radiosensitivty of a target tumour by a factor of 1.2-5 [36]. Due to these beneficial properties, HT can prove highly advantageous when combined with radiation or chemotherapy in clinical applications [36].

Additionally, HT can be used to facilitate the active targeting of tumour cells with chemotherapy drugs encapsulated in specialized drug carriers such as thermosensitive liposomes (TSLs) [37]. The

use of HT as an externally applied trigger for thermosensitive drug carriers shows strong potential, as the subsequent increase in temperature of the drug target improves permeability and drug uptake in the ROI [38, 39]. This process allows the sparing of healthy organs around the target and lowers 
treatment toxicity [40]. An illustration of the HT driven TSL drug release is depicted in Figure 1.5. Here a heating source is used to increase the temperature of a ROI to within the HT regime.
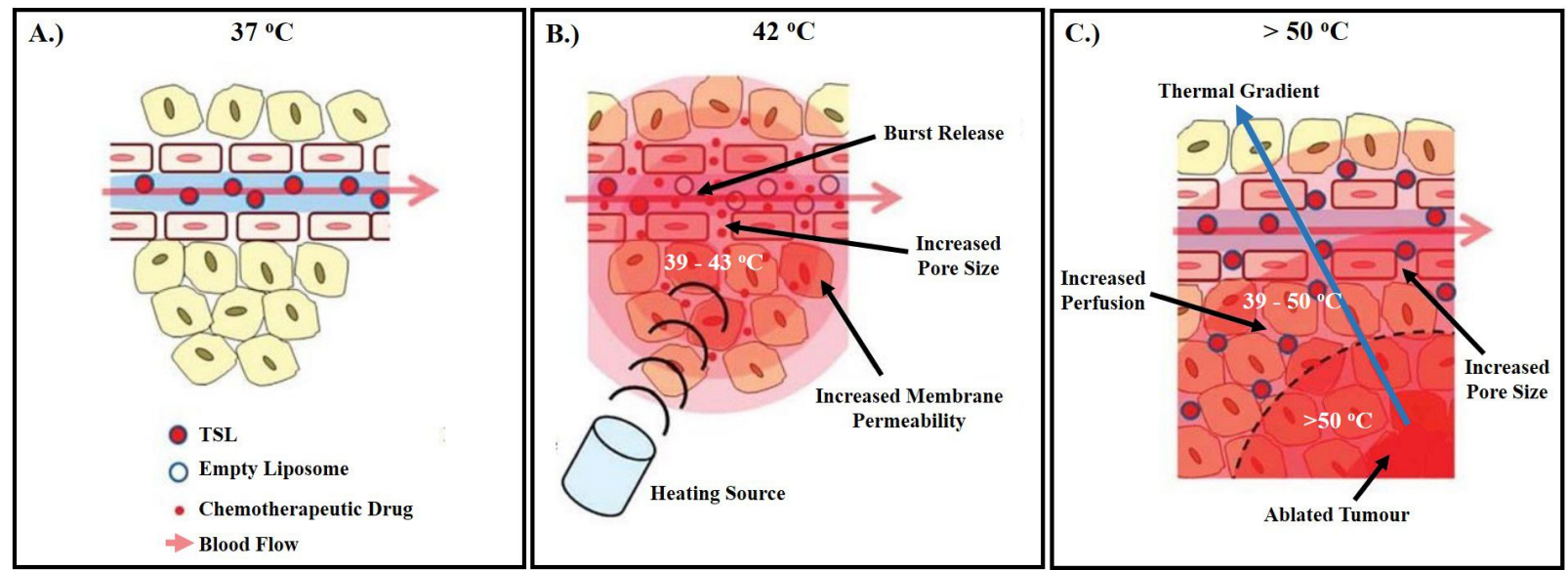

Figure 1.5: (A) A chemotherapeutic drug is encapsulated in TSLs and passively carried to the tumour site. (Middle) The use of HT with TSL drug carriers allows the burst release of a chemotherapeutic drug into surrounding tumour tissue at the ROI only. (C) Thermal ablation of the tumour core causes localized coagulation necrosis, and drug release near the tumour boundaries. (Figure adapted from May et al. [37]).

Generally, HT is combined with radiation or chemotherapy and has a relatively longer treatment time than thermal ablation, which is on the scale of minutes. In thermal ablation therapy a heating apparatus is used to rapidly heat a tumour. Unlike the HT approach, thermal ablation is most often used as a stand alone modality and not in conjunction with other treatments [41]. By heating a ROI to high temperatures above $45^{\circ} \mathrm{C}$ coagulation necrosis is induced, leading to irreversible cell death in the target. In practice, ablating tumours with a large volume or irregular shapes can be problematic with this technique. Much like HT, thermal ablation can be used for active targeting with TSLs. In this case, the tumour core could be coagulated $\left(T>55^{\circ} \mathrm{C}\right)$ while the surrounding tissue would be heated at lower temperatures $\left(\approx 42^{\circ} \mathrm{C}\right)$, becoming more susceptible to chemotherapy agents [37].

\subsubsection{Available Thermotherapy Modalities}

There are a variety of thermotherapy modalities currently available with some more established methods including microwave, laser, gold nanoparticle (GNP), radiofrequency (RF), and ultrasound thermotherapy. In general, all available modalities are derived from one of three basic mechanisms 
of heat delivery: the thermal conduction of heat, resistive or dielectric loss from an applied electromagnetic field, or the mechanical loss of energy due to molecular collisions from an ultrasound wave [32]. Each modality has unique advantages, limitations, and applications which will be discussed in this section.

\section{Microwave Thermotherapy}

In microwave thermotherapy, either a single interstitial microwave antenna or an array of microwave antennas can be placed within deep set tumours in the body. These antenna serve as sources of microwave radiation, and can initiate large thermal lesion formation in the target through the deposition of microwave radiation. By using an array of microwave antennas to ablate a target, irregularly shaped and large volume tumours can also be treated [42]. One distinct advantage of microwave thermotherapy is the ability of microwave energy to propagate through desiccated and charred tissue. This allows the potential to ablate a larger area around the target compared to other thermotherapy modalities [43]. As a result, treatment with a microwave array can prove advantageous when treating large area chest wall disease in combination with external beam radiation, chemotherapy, or in some cases brachytherapy. One disadvantage of this modality is that the relatively long treatment time can lead to skin burns, which can be minimized with the use of a water bolus during treatment [44].

\section{Laser Thermotherapy}

Due to the high scattering and corresponding low penetration depth of laser photons in tissue, laser thermotherapy is primarily used to deal with superficial tumours and has become an integral part of dermatological therapy [45]. In cases of deep set tumours, fiber-optic catheters can also be utilized in a procedure known as laser interstitial thermal therapy (LITT) to deliver the laser photons. In this minimally invasive technique a fiber optic catheter is stereotactically guided to deliver energy to a ROI, resulting in selective thermal ablation of lesions [29].

LITT involves the creation of a small cranial burr hole under minimal sedation or general anesthesia. A thin optical fiber is then inserted into the brain and moved to the ROI via MRI guidance to a precision of $1 \mathrm{~mm}$ or less [46]. Here LITT provides a distinct advantage over a 
standard craniotomy as the tumour can be reached and treated without the need for removal of the bone flap. To treat the tumour a low voltage laser is used to excite chromophores within the ROI. Molecular excitation then leads to the subsequent release of thermal energy and temperature increase of the tissue [47]. By manipulating laser pulse length and heating time, the desired thermal effect can be set such that thermal ablation is achieved evenly around the probe tip. Since the absorption coefficient $\left(\mu_{a}\right)$ of pathologic lesions is higher than that of healthy brain parenchyma, preferential ablation of tumour tissue can be achieved [48]. This can allow the selective ablation of tumour tissue with a minimally-invasive procedure compared to conventional resection.

As of now LITT has been successfully used to treat a number of different brain tumours, ranging from resistant metastatic tumours, to astrocytomas, chordomas, meningiomas, and glioblastomas [48]. In these cases, curative effects similar to those with open surgical procedures were achieved in a minimally invasive manner [46]. One limitation of this modality is the inability to spare healthy tissue between the laser probe and the pathologic tissue target. This can be avoided using LITT, however the procedure is not completely noninvasive [29, 49].

\section{Gold Nanoparticle Thermotherapy}

In GNP thermotherapy gold nanoparticles are used to facilitate precision targeting of a ROI. In this technique GNPs are synthesized with a dielectric silica core and a ratio of core diameter to shell thickness such that they effectively absorb light in the infrared region. This modality utilizes lasers with wavelengths ranging from 600 to $1400 \mathrm{~nm}$, where absorption in soft tissue is low, to target embedded GNPs in a ROI [50]. If the wavelength of the laser used is near the plasmon-resonant band of the embedded GNP, a temperature increase can be induced with a $1-5 \mathrm{~W} / \mathrm{cm}^{2}$ laser after a few minutes of exposure [51].

To achieve selective heating of tumour tissue, a near infrared laser pulse is absorbed by the GNPs to excite free electrons in the plasmon band. These hot electrons then cool rapidly through interactions with the surrounding gold lattice, heating it to high temperatures. Heat is then transferred to the surrounding tissue on the time scale of $\approx 100 \mathrm{pS}$, causing a rapid temperature increase on the scale of tens of degrees [52, 53]. This process allows highly localized heating of a tumour volume to be achieved. At this time GNP thermotherapy is still experimental, and more work is required to 
make this modality clinically feasible [54].

\section{Radiofrequency Thermotherapy}

$\mathrm{RF}$ thermotherapy is a more commonly used minimally invasive modality which relies on the conductivity of tissue to allow heating of a ROI. This heating modality operates using either single interstitial electrodes (monopolar method) or two parallel surface electrodes (bipolar method) [55]. In either case a closed loop circuit is created with the needle electrode, a dispersive ground electrode or grounding pad, and the patient in series. In this series both the needle and ground electrodes are active, while the patient acts as a resistor with relatively high resistance compared to the electrodes. When an RF field is applied to the electrodes an alternating electric field is created within the patient. This causes agitation of ions present within tumour tissue close to the electrodes, and raises tissue temperature through friction [55, 56].

In order to treat large deep set tumours RF fields with large wavelengths compared to the target tissue (10-120 MHz) are often used. By manipulating the frequency of RF fields used, the amount of thermal deposition can be accurately controlled [56]. Unfortunately, as tissue is coagulated its resistance to current flow is increased. This can impede the heating of tissue and RF thermal lesion size when using this modality on larger tumours [56].

\section{Ultrasound Thermotherapy}

Although used extensively as an imaging modality, ultrasound has also seen significant development as a noninvasive therapeutic tool. In ultrasound thermotherapy, tissue heating is the result of the absorption of ultrasonic energy. Here mechanical oscillations propagate through a target medium and deposit energy at a set focal point as heat, while a fraction of the total energy is lost as attenuation [30, 57]. When compared with other thermotherapy modalities, ultrasound has proven highly advantageous, primarily due to its noninvasive nature, high penetration depth in soft tissue, and high degree of beam control [58].

Ultrasound thermotherapy can be classified based on the power of the therapy transducer as either "high power" or "low power" treatments. Low power treatments include HT, while high power treatments involve the use of high intensity focused ultrasound (HIFU) transducers to achieve 
noninvasive ablation of a target [59]. In HIFU treatment the intensity of the ultrasound beam is high and focused onto a small focal point. As an analogy, consider the focusing of sun rays with a magnifying glass to start a fire. HIFU operates on roughly the same principles, with the focusing of ultrasound waves rather than light [58]. HIFU treatment can be performed from outside the patients body, and rapidly increases temperature at the focal point over a very short exposure time. This induces coagulative necrosis and subsequent cell death in the target, with little thermal effect on healthy tissue both surrounding the ROI and in the path of the ultrasound beam [60, 61]. In HIFU treatment an additional mechanism of tissue damage, known as acoustic cavitation, must also be considered. In this process pre-existing microbubbles in the ROI are excited and collapse, releasing energy into the surrounding medium [60].

HT can also be performed with therapeutic ultrasound transducers operated at lower power and intensity. This low intensity focused ultrasound (LIFU) therapy can heat a target ROI to the hyperthermia temperature regime after a few of minutes of exposure. In LIFU therapy the absorption of ultrasonic waves at the focal point is the dominant mechanism of ultrasound-tissue interaction, and acoustic cavitation is generally not considered.

Compared to other thermotherapy modalities ultrasound can prove beneficial due to its sharp and tailored treatment margins, however there are limitations which must also be considered. Due to the high reflection of ultrasound waves from gas or bony structures, there is the possibility for complications when dealing with liver treatment near the rib cage or gas containing bowels. In these cases the high energy ultrasound waves can reflect at the soft tissue-bone-or-gas interface and deposit energy in healthy tissue, leading to a failure to satisfy the prescribed thermal dose and patient pain [58].

At this time there are two primary types of clinical ultrasound devices available for extracorporeal and transrectal thermotherapy. Extracorporeal treatment has a focal length of roughly 10 to $16 \mathrm{~cm}$ and can be used to heat targets within the abdomen, such as the kidney, liver, breast, soft tissue, or pancreas. Transrectal treatment has a shorter focal length of 3 to $4 \mathrm{~cm}$, and is used primarily to treat the prostate $[58,59,60,62]$. 


\subsubsection{Noninvasive Thermometry}

Due to the temperature sensitive nature of thermotherapy, as well as the effects of tissue heterogeneity on heat distribution in a ROI, it is imperative that a reliable form of real-time thermometry is available to ensure success and safety in clinical thermotherapy. A robust thermometry technique must accurately monitor the extent of thermal destruction throughout the ROI to a high degree of accuracy, as an increase of only a few degrees can mean the difference between HT and thermal ablation treatment. For clinical thermotherapy a thermocouple or fiber-optic sensor measurement is not ideal, as thermocouples are invasive, yield only a point measurement, and can have a nonnegligible effect on the thermal field in the ROI. As a result, there is strong motivation for the development of a reliable form of noninvasive thermometry [63, 64, 65]. A variety of potential noninvasive modalities have been proposed based on both magnetic resonance (MR) and ultrasound imaging techniques to satisfy this [63,66].

\section{MR Thermometry}

In noninvasive thermometry the current gold standard is that of magnetic resonance (MR) thermometry. MR thermometry shows great potential as a noninvasive thermometry technique as it is non-ionizing and has a number of temperature sensitive parameters, including proton density, $T_{1}$ and $T_{2}$ relaxation times, the diffusion coefficient, magnetization transfer, and proton resonance frequency (PRF) [66]. The most commonly used MR thermometry technique is the PRF shift method, as it has shown high sensitivity, good temporal resolution, and is independent of tissue type and thermal history with the exceptions of adipose or coagulated tissue [66].

The working mechanism behind the PRF shift method is the measurement of changing resonance frequency of water protons $(\omega)$ within the ROI as it is heated. The resonance frequency of a proton in a molecule can be expressed as follows:

$$
\omega=\gamma B_{\text {local }}=\gamma(1-s) B_{0}
$$

Here $\gamma$ represents the gyromagnetic ratio of the proton, $B_{\text {local }}$ is the local magnetic field at the nucleus of the molecule, $s$ is a screening constant dependant on the chemical environment, and $B_{0}$ 
is the magnetic flux density [66]. During thermotherapy the temperature of the ROI is increased, causing hydrogen bonds within the ROI to bend and break. The breaking of hydrogen bonds, and subsequent increase in the presence of free $\mathrm{H}_{2} \mathrm{O}$ molecules, then leads to a more uniform distribution of electron clouds and a subsequent increase in electron screening of hydrogen nuclei within the ROI. As seen in Equation 1.2, an increase in screening will result in a decrease in measured PRF. By comparing with a reference image (before heating) the PRF shift can then be directly related to temperature change in the ROI [66]. The calculated temperature change can then be correlated with computer models of predicted cell death to provide a real-time quantitative measurement of tissue necrosis [67].

Current clinical applications of image guided thermotherapy include MR-guided LITT (MRgLITT) for the treatment of brain tumours, and MR-guided focused ultrasound (MRgFUS) primarily for the treatment of uterine fibroids. In both cases, the use of MR guidance allows the selective thermal ablation of pathological tissue with a sharp thermal border between the tumour and healthy tissue [67, 68]. Although the PRF shift thermometry method is accurate and noninvasive, it is expensive, non-portable, and requires the use of MR compatible heat applicators during treatment. In current research a number of noninvasive real time thermometry techniques are being developed to address this limitation. One promising noninvasive thermometry modality is that of ultrasound thermometry [63].

\section{Ultrasound Thermometry}

Although ultrasound thermometry has not been routinely implemented in the clinic, three major temperature sensitive ultrasound parameters have been proposed for this purpose. These include the change in attenuation, the change in backscattered energy $(\mathrm{BE})$, and the backscattered RF echo shift. Here the RF echo shift refers to the apparent displacement of ultrasound speckle due to the temperature dependant change in speed of sound and thermal expansion of the medium [63, 69]. Additionally, there are temperature dependant parameters of nonlinearity which describe the degree of acoustic harmonic production in nonlinear wave propagation. The magnitude of produced acoustic harmonics have also been shown to exhibit temperature dependence [24]. 


\section{Change In Attenuation Coefficient:}

During propagation of ultrasound waves through a medium, the wave form can be manipulated both by nonlinearity of the medium and frequency dependant attenuation [21]. At boundaries in a nonlinear medium a fraction of the initial wave is transmitted through, while the remaining fraction is reflected. The amplitude of the transmitted wave is dependant on the characteristic impedance on either side of the boundary. Attenuation is a measurement of the absorption and scattering of the ultrasound wave as it propagates through the medium, resulting in a loss of energy and wave amplitude. This can be represented mathematically using the exponential law of attenuation as follows [21]:

$$
I(x)=I(0) e^{-2 \alpha x}
$$

Here $I(x)$ and $I(0)$ represent average signal intensity at some location $x$ and $x=0$ (initial intensity) respectively, while $\alpha$ is known as the attenuation coefficient. Here $\alpha$ is a function of both temperature and frequency. The frequency dependence of the attenuation coefficient for most soft tissues can be modelled as follows [21]:

$$
\alpha(f)=\alpha_{0} f^{n}
$$

Here $\alpha_{0}$ is the attenuation coefficient at $1 \mathrm{MHz}$, and $n$ is a frequency dependence constant such that for soft tissue $1 \leq n \leq 2$. From this relationship it is evident that for higher frequencies the wave will experience a higher degree of attenuation.

The effects of temperature on the attenuation coefficient in soft tissue has been widely investigated in the past, both in the HT and thermal ablation regime. However, as stated by Bamber, "no single absorption or scattering mechanism has yet been used to explain the observed temperature dependence of attenuation in soft tissues" [70]. Although further research is needed to accurately determine temperature dependence of $\alpha$, it has been shown that at temperatures above $50^{\circ} \mathrm{C}$ an increase in $\alpha$ is seen due to coagulation necrosis. This results in irreversible structural change in the tissue and a corresponding permanent increase in $\alpha$ [71, 72]. 


\section{Echo-Shift Technique:}

Arguably the most established noninvasive ultrasound thermometry technique is that of measuring backscattered RF echo-shift. The working mechanism behind this method is that of temperature dependant change in SOS, which causes fluctuations in scatterer location; and the thermal expansion of the medium, which causes apparent fluctuations in scatterer location [73, 74]. In the HT regime the effects of the change in SOS on measured echo-shift dominate over thermal expansion [75]. Assuming a small-amplitude (no acoustic harmonics) signal the SOS (c) can be expressed mathematically as a function of adiabatic compressibility $(\kappa)$, and the density of the medium at equilibrium $\left(\rho_{0}\right)$ as follows:

$$
c=\sqrt{\frac{1}{\kappa \rho_{0}}}
$$

As a ROI is heated SOS increases to a maximum value of $1557 \mathrm{~m} / \mathrm{s}$ at $74^{\circ} \mathrm{C}$ followed by a gradual decrease [21]. $\kappa$ displays an opposite trend, decreasing as temperature is increased until reaching a minimum at $66^{\circ} \mathrm{C}$ followed by a gradual increase [21].

Assuming that the SOS is linearly proportional to temperature, the change in temperature can be found by first measuring the shift in RF echo at each location of the medium between two consecutive frames with standard cross correlation techniques, and then differentiating them along the axial direction with a tissue dependant scaling factor. The change in temperature at some depth $z$ can then be represented mathematically as follows [74]:

$$
\delta T(z)=\left[\frac{c_{0}}{2}\left(\frac{1}{\alpha_{L}-\lambda}\right)\right]\left[\frac{d}{d z}(\delta t(z))\right]
$$

Here $\lambda\left[{ }^{\circ} \mathrm{C}^{-1}\right]$ is the thermal coefficient of the SOS, $\alpha_{L}\left[{ }^{\circ} \mathrm{C}^{-1}\right]$ is the linear coefficient of thermal expansion of the medium, and $\delta t(z)$ is the cumulative time-shift at some depth $z$. The first term in Equation 1.6, which is sometimes simplified as $K$, is a material dependent parameter. The second term represents the axial gradient of the cumulative shifts in the measured RF echo. Here the cumulative time shift is found from summing all frame-to-frame incremental time shifts between successive RF frames.

One major obstacle that must be overcome before the implementation of this technique in in 
vivo applications is that of respiratory and cardiac motion, as tissue motion during treatment can cause the temporal shifts due to temperature changes being missed.

\section{Change In Acoustic Harmonics:}

Another promising noninvasive thermometry technique is that of monitoring the temperature dependant change in generated acoustic harmonics during nonlinear wave propagation. As previously mentioned (see section 1.1.2), during propagation of ultrasound waves through a medium the wave form can be manipulated both by nonlinearity of the medium and frequency dependant attenuation [21, 22]. This leads to the progressive distortion of the wave as it propagates, and the generation of secondary higher frequency signals known as acoustic harmonics.

The amount of nonlinearity of a specific medium can be quantified by a temperature dependant parameter known as the parameter of nonlinearity $(B / A) . B / A$ is the ratio of coefficients of the quadratic and linear terms in the Taylor series expansion which relates variations in acoustic pressure to variations in density of a medium. $B / A$ can be derived by first considering the pressure-to-density relation for liquids as an equation of state:

$$
P=P(\rho, s) \quad\left\{\begin{array}{c}
P=P_{0}+P_{1} \\
\rho=\rho_{0}+\rho_{1}
\end{array}\right.
$$

Here $s$ is entropy, $P$ is pressure, and $\rho$ is density, with 0 and 1 subscripts denoting equilibrium and perturbed values respectively. From this a Taylor series expansion can be applied to the equation of state under adiabatic conditions such that:

$$
P-P_{0}=\left(\frac{\partial P}{\partial \rho}\right)_{s, \rho_{0}}\left(\rho-\rho_{0}\right)+\frac{1}{2 !}\left(\frac{\partial^{2} P}{\partial \rho^{2}}\right)_{s, \rho_{0}}\left(\rho-\rho_{0}\right)^{2}+\frac{1}{3 !}\left(\frac{\partial^{3} P}{\partial \rho^{3}}\right)_{s, \rho_{0}}\left(\rho-\rho_{0}\right)^{3}+\cdots
$$

By evaluating all partial derivatives at the equilibrium state and constant entropy, equation 1.8 can be simplified as follows:

$$
P_{1}=A\left(\frac{\rho_{1}}{\rho_{0}}\right)+\frac{B}{2 !}\left(\frac{\rho_{1}}{\rho_{0}}\right)^{2}+\frac{C}{3 !}\left(\frac{\rho_{1}}{\rho_{0}}\right)^{3}+\cdots
$$

Here $A, B$, and $C$ represent the linear, quadratic, and cubic coefficients of the Taylor series 
expansion and can be expressed as follows (here $c_{0}$ is the wave velocity):

$$
A=\rho_{0}\left(\frac{\partial P}{\partial \rho}\right)_{s, \rho_{0}} \equiv \rho_{0} c_{0}^{2} \quad, \quad B=\rho_{0}^{2}\left(\frac{\partial^{2} P}{\partial \rho^{2}}\right)_{s, \rho_{0}} \quad, \quad C=\rho_{0}^{3}\left(\frac{\partial^{3} P}{\partial \rho^{3}}\right)_{s, \rho_{0}}
$$

From this, $B / A$ can be found as the ratio of quadratic and linear coefficients:

$$
\frac{B}{A}=\frac{\rho_{0}}{c_{0}^{2}}\left(\frac{\partial^{2} P}{\partial \rho^{2}}\right)_{s, \rho_{0}}
$$

By expanding this derivative, a final equation can be obtained demonstrating the temperature dependence of $B / A$. Here $c$ is the measured SOS at a given temperature $(T)$ and pressure $(P), \alpha_{p}$ is the isobaric thermal expansion coefficient, and $c_{p}$ is the isobaric specific heat capacity [76]:

$$
\frac{B}{A}=2 \rho_{0} c_{0}\left(\frac{\partial c}{\partial P}\right)_{T, \rho_{0}}+2 c_{0} T \alpha_{p} c_{p}^{-1}\left(\frac{\partial c}{\partial T}\right)_{P, \rho_{0}}
$$

Using $B / A$ the phase velocity $\left(v_{\theta}\right)$ of a wave during nonlinear propagation can be also be expressed as a function of $c_{0}$ and particle velocity $(v)$ [77]. This demonstrates that the phase velocity is not constant in the case of nonlinear wave propagation:

$$
\begin{aligned}
v_{\theta}=c_{0}+v+\left(\frac{B}{2 A}\right) v & =c_{0}+\left(1+\frac{B}{2 A}\right) v=c_{0}+\beta v \\
\boldsymbol{\beta} & =\mathbf{1}+\frac{\boldsymbol{B}}{\mathbf{2 A}}
\end{aligned}
$$

Here $\beta$ is known as the coefficient of nonlinearity, and represents the nonlinear correction in phase velocity due to the influence of nonlinear effects caused by the propagation of a finite amplitude wave [76]. Two primary methods exist for measuring $B / A$. The first, known as the thermodynamic method, allows $B / A$ to be evaluated based on the change in SOS with respect to both pressure and temperature as shown in Equation 1.12. The second method, known as the finite-amplitude method, is based on the analysis of waveform distortion due to harmonics generation during wave propagation. Using these methods the temperature dependence of $B / A$ has been demonstrated experimentally in both porcine fat and liver tissue [78]. One distinct advantage of the acoustic harmonic method over other noninvasive ultrasound thermometry techniques such as echo-shift is 
the lack of sensitivity to tissue motion. This can prove advantageous when dealing with cardiac and respiratory motion in in vivo studies [79]. Since the amplitude of produced acoustic harmonics are dependant on $B / A$ and $\beta$, they also exhibit a temperature dependence and the potential for use as a thermometry parameter [73, 79].

\section{Change In Backscattered Energy:}

The change in backscattered energy (CBE) can be measured experimentally as the change in RF echo BE between consecutive frames as a ROI is heated or cooled. To calculate BE, the envelope of each signal was found using the Hilbert transform and all envelope values squared [73, 80]. The magnitude of $\mathrm{CBE}$ with changing temperature is determined primarily from the tissue type and density, and can show a positive or negative slope depending on tissue homogeneity [80, 81].

In previous work by Maraghechi et al. noninvasive thermometry based on CBE was also paired with acoustic harmonic thermometry, through measuring $\mathrm{CBE}$ of individual acoustic harmonics as a sample was heated. Here the change in backscattered energy of each harmonic signal $(h \mathrm{CBE})$ at some time, $\tau$, was calculated relative to a baseline BE measurement at a reference temperature, $B E(0)$, as follows:

$$
h \operatorname{CBE}(\tau)=\left[\frac{B E(\tau)-B E(0)}{B E(0)}\right] \times 100
$$

From this, $h \mathrm{CBE}(\tau)$ was plotted as a $2 \mathrm{D}$ colormap showing $h \mathrm{CBE}$ as a function of sample temperature.

Using this technique, Maraghechi et al. successfully produced 2D $h$ CBE maps for both tissuemimicking gel phantoms and ex vivo bovine muscle tissue as the samples were heated and cooled. For both the phantom and ex vivo tissue trials a nonlinear single frequency (NLSF) imaging method was used to produce both B-mode images and 2D $h \mathrm{CBE}$ maps for the $h_{1}, h_{2}$, and $h_{3}$ signals as a function of temperature. In both the phantom and ex vivo tissue trials the sample was heated via hot water channel circulation from $38^{\circ} \mathrm{C}$ to $46^{\circ} \mathrm{C}$, with measurements of $h \mathrm{CBE}$ performed at a transmit frequency of $13 \mathrm{MHz}$ every $2^{\circ} \mathrm{C} .2 \mathrm{D}$ maps of $h \mathrm{CBE}$ relative to a baseline measurement at $38^{\circ} \mathrm{C}$ were then generated, showing an increase in $h \mathrm{CBE}$ as the sample was heated, and a decrease in $h \mathrm{CBE}$ as the sample was allowed to cool [82]. 
From the generated $h \mathrm{CBE}$ maps, a $h \mathrm{CBE}$-to-temperature calibration was attempted, however standard deviations of $h \mathrm{CBE}$ and temperature were high [73]. It was concluded that the SNR of the produced $h \mathrm{CBE}$ maps could be improved in order to reduce uncertainty in the $h \mathrm{CBE}$-to-temperature calibration curve [73].

\subsection{Thesis Hypothesis and Specific Aims}

In this work, the techniques of both frequency compounding and THI were combined in a new nonlinear frequency compounding (NLFC) method. By both reducing speckle through frequency compounding, and utilizing the advantages of THI, the overall image quality of B-mode and BE images was improved, allowing a hCBE-to-temperature calibration to be performed

Using the NLFC method ultrasound B-mode and BE imaging were generated of a calibrated tissue mimicking phantom with the objective of increasing image quality over the previously used NLSF method. For all calibrated phantom B-mode and BE images speckle signal-to-noise ratio (SSNR) was used as a metric for image quality to quantify the improvement over the NLSF as a result of the new NLFC method.

The NLFC method was then applied to $h \mathrm{CBE}$ based noninvasive thermometry, and used to perform a $h \mathrm{CBE}$-to-temperature calibration. Lastly, 2D maps of temperature for a heated ex vivo porcine tissue sample were produced using both the NLFC and NLSF methods and compared with a theoretical COMSOL based model. The hypothesis of this thesis is that by developing a NLFC method SSNR of both B-mode and BE images can be improved over the previously used NLSF method, and a $h \mathrm{CBE}$-to-temperature calibration for use in tissue thermometry can be performed.

The main contributions of this work can be classified in two parts as follows: (1) The development of a NLFC method and optimization of input parameters based on nonlinear ultrasound B-mode and BE imaging in a tissue mimicking phantom. (2) The development and application of the NLFC method in nonlinear ultrasound thermometry in ex vivo tissue. The materials and experimental procedure used in both contributions are are outlined in Chapter 2. Experimental results and discussion for both contributions are presented in Chapter 3. Lastly, a general overview and discussion on the improvements in B-mode and BE image quality, as well as the generation of 
$h \mathrm{CBE} 2 \mathrm{D}$ maps due to the new NLFC method is provided in Chapter 4.

The specific aims of this thesis were as follows:

1. Develop a NLFC method to generate frequency compounded B-mode and BE images and determine optimal input parameters by maximizing SSNR in a calibrated tissue mimicking phantom.

2. Perform localized heating of ex vivo porcine tissue with a clinically relevant heating modality in the form of a therapeutic LIFU transducer, and generate 2D maps of $h$ CBE.

3. Perform a $h \mathrm{CBE}$-to-temperature calibration using the NLFC method and generate $2 \mathrm{D}$ temperature maps for localized LIFU heating of ex vivo porcine tissue.

4. Perform a comparative study on the NLFC and previous NLSF methods and quantify the improvement in SSNR of B-mode and BE images of a tissue mimicking phantom, SNR of ex vivo porcine tissue $h \mathrm{CBE}$ maps, as well as the agreement of $2 \mathrm{D}$ NLFC temperature maps with a theoretical COMSOL based model. 


\section{$2 \mid$ Materials and Methods}

\subsection{Development and Optimization of The NLFC Method In Nonlinear B-Mode and BE Imaging}

In order to improve image quality of both ultrasound B-mode and BE images a novel NLFC method was developed to perform nonlinear frequency compounding of both $h_{1}$ and $h_{2}$ signals. Optimized input parameters were then determined for the NLFC method to maximize SNR in frequency compounded B-mode and BE images of a calibrated tissue mimicking phantom. In this section a step-by-step comparison of both the NLFC and previous NLSF methods will be provided, as well as an overview of the NLFC optimization process. Lastly, the improvement in SSNR of the NLFC B-mode and BE images over the previously used NLSF method will be quantified.

In this work both the NLSF and NLFC methods were performed using a 256 element linear array transducer (L12-5 50mm, Philips, Amsterdam, Netherlands) paired with a Verasonics ${ }^{\circledR}$ ultrasound research system (Vantage 3.4, Verasonics ${ }^{\circledR}$ Inc., Kirkland, Washington, United States). The frequency response of the L12-5 imaging transducer is provided in Figure 2.1. All B-mode and BE images were generated using a tissue mimicking calibrated phantom (CIRS Model 040GSE Multi-Purpose Multi-Tissue Ultrasound Phantom, Universal Medical, Oldsmar, Florida, United States). The tissue mimicking phantom (TMP) used for measurements had an attenuation of $0.5 \mathrm{~dB} / \mathrm{cm}$ and SOS of $1540 \mathrm{~m} / \mathrm{s}$. 


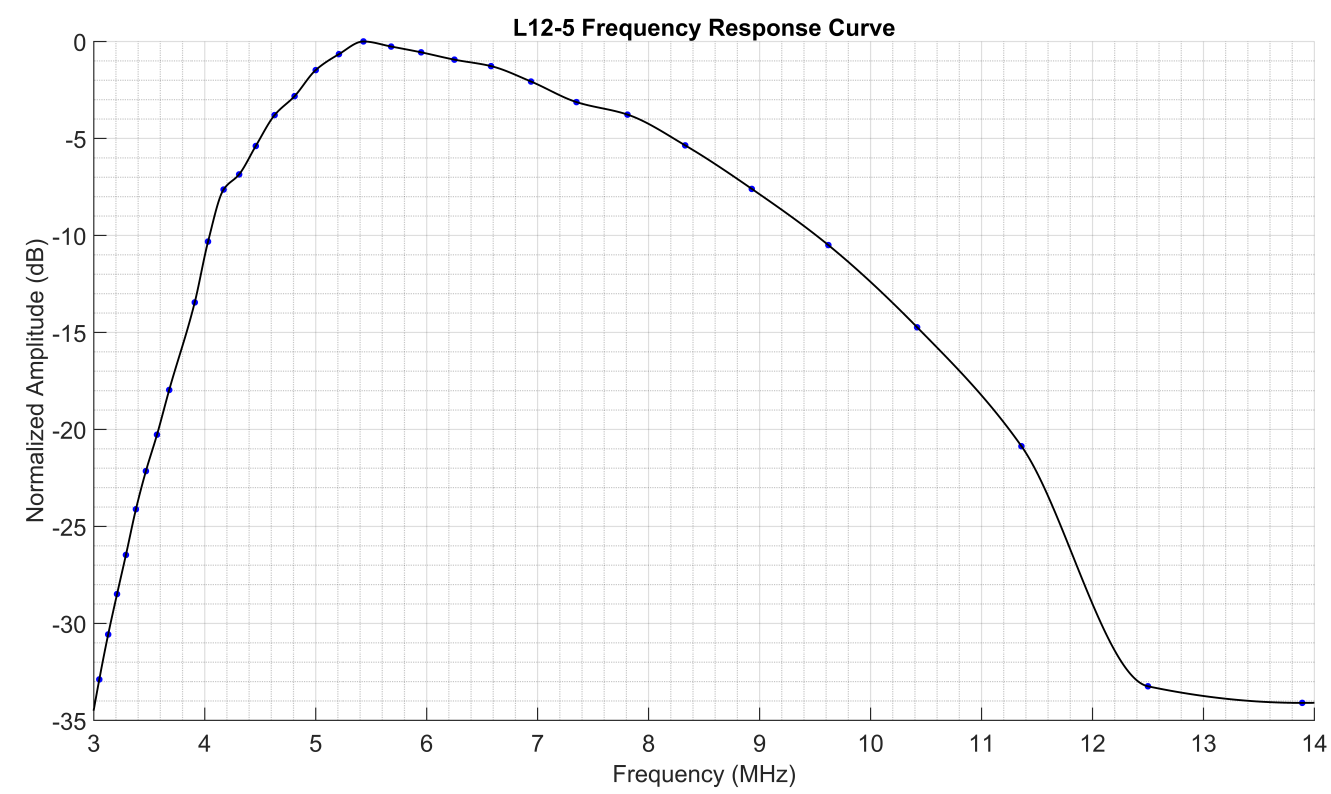

Figure 2.1: To measure the bandwidth of the L12-5 imaging transducer a flat reflector was placed at the focus of the transducer, and the reflected signal was measured when the transducer was excited by a 4-cycle pulse at various transmit frequencies.

\subsubsection{NLSF Method}

The NLSF process begins with the transmission of an initial pulse from the L12-5 (256 channels) linear array transducer $(p)$, and a subsequent received signal $(s)$. Using MATLAB (MathWorks, Natick, MA), the saved signal was then beamformed and the Fourier transform $(\mathcal{F})$ applied to switch to the frequency domain. Note that here $t$ and $\omega$ denote time and frequency, while $S$ is the signal in the frequency domain:

$$
\begin{gathered}
p(t) \underset{\text { Signal received }}{\stackrel{\text { Pulse transmitted }}{\longrightarrow}} s(t) \\
\mathcal{F}\{s(t)\}=S(\omega)
\end{gathered}
$$

In order to separate the $h_{1}$ and $h_{2}$ signals, two Gaussian filters were then applied. The $h_{1}$ filter $\left(g_{1}\right)$ was centered at the transmit frequency with a percent bandwidth of $60 \%$, while the $h_{2}$ filter $\left(g_{2}\right)$ was centered at double the transmit frequency with a width of $22 \%$ percent bandwidth. Note that here, percent bandwidth is defined as the absolute bandwidth divided by the center frequency of the 
filter. These filter percent bandwidths were selected to ensure that a sufficient absolute bandwidth was available to capture the entirety of the $h_{1}$ and $h_{2}$ signals, while simultaneously avoiding filter overlap at a low number of pulse cycles (2-12 cycles were tested while optimizing NLFC method input parameters in this work). An example of the filtering process is shown in Figure 2.2 for a single $S(\omega)$ signal.
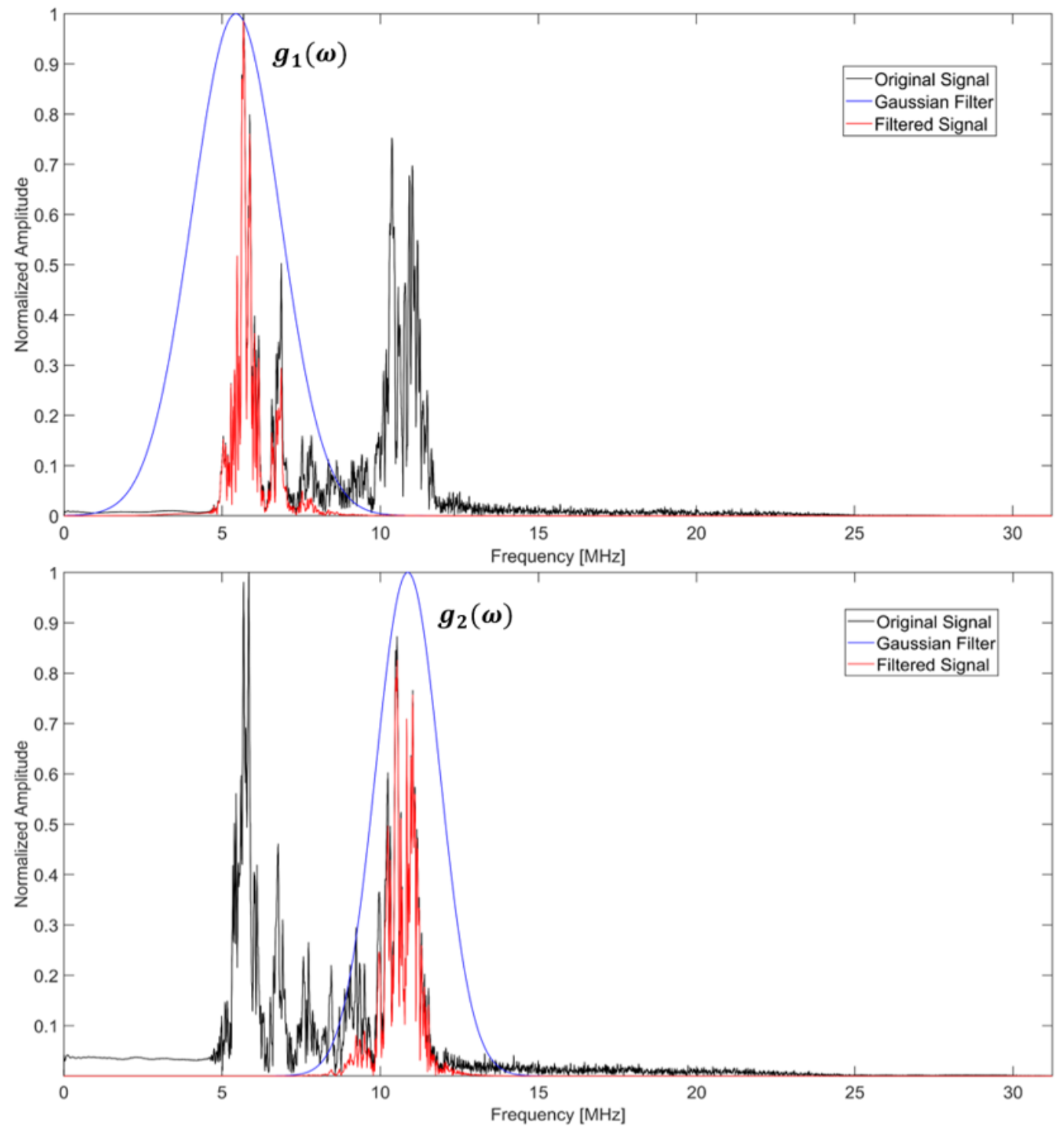

Figure 2.2: (Top) The $g_{1}(\omega)$ Gaussian filter is applied to extract the fundamental signal. (Bottom) The $g_{1}(\omega)$ filter is applied to extract the second harmonic signal. Here the original unfiltered signal is shown in black, while the Gaussian filter and produced filtered signal are shown in blue and red respectively. 
The filtering process was performed for $256 \mathrm{RF}$ lines each for the $h_{1}$ and $h_{2}$ signals, and the inverse Fourier transform $\left(\mathcal{F}^{-1}\right)$ applied to each RF line to return to the time domain as follows:

$$
\begin{aligned}
& s_{1}(t)=\mathcal{F}^{-1}\left\{S(\omega) \times g_{1}(\omega)\right\} \\
& s_{2}(t)=\mathcal{F}^{-1}\left\{S(\omega) \times g_{2}(\omega)\right\}
\end{aligned}
$$

By combining all 256 filtered signals $(x, y)$ frames for the filtered $h_{1}$ signals $\left(S_{1(x, y)}\right)$, and filtered $h_{2}$ signals $\left(S_{2}(x, y)\right)$ were then found. A schematic of a typical $S_{1}(x, y)$ or $S_{2}(x, y)$ frame is provided in Figure 2.3. Here a time signal, with axial depth on the $\mathrm{x}$-axis and magnitude in arbitrary units on the y-axis, is generated through the process outlined in Equations 2.3 and 2.4 for $256 s(t)$ signals. These 256 signals were then plotted in frame form as a function of channel number $(x)$ and axial depth $(y)$. For these experiments the sampling frequency was set in the y-axis direction such that all $S_{1}(x, y)$ and $S_{2}(x, y)$ frames had dimensions of $256 \times 5323$.

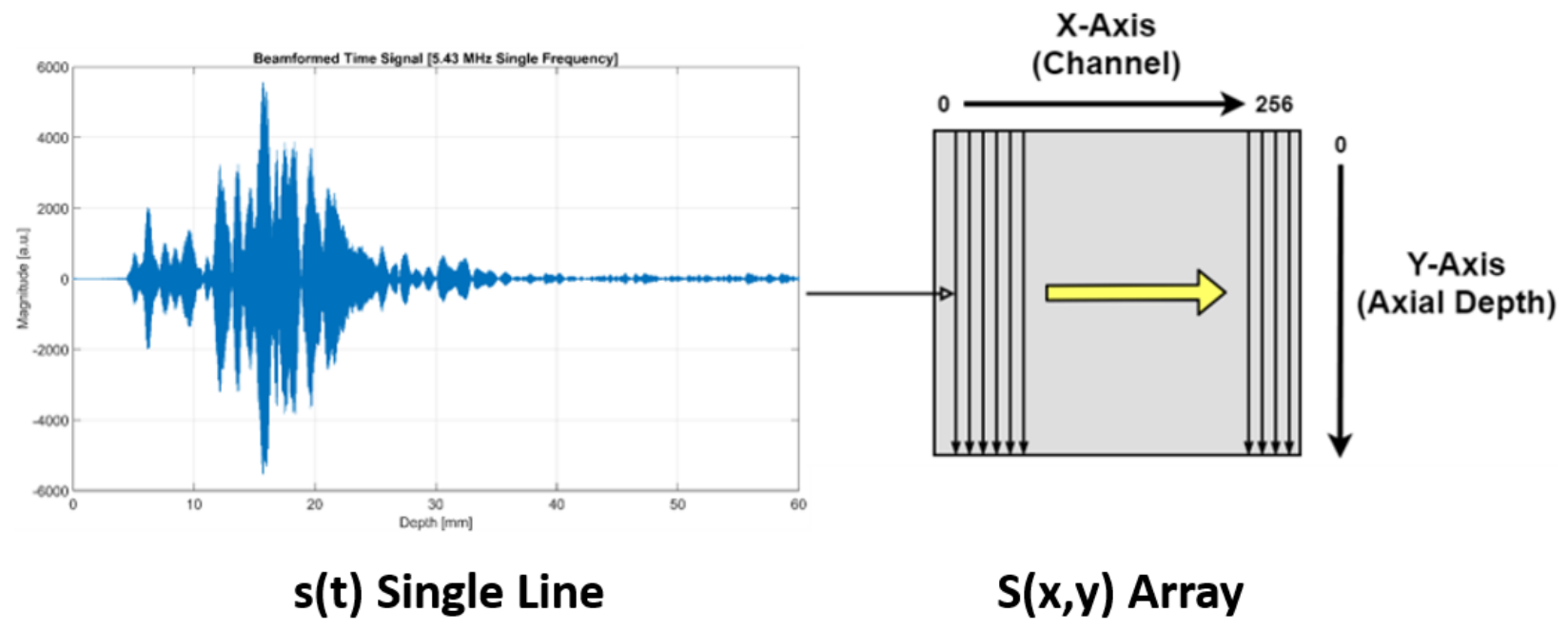

Figure 2.3: (Left) A typical time signal obtained from multiplying a Gaussian filter to a single $S(\omega)$ signal, and applying the inverse Fourier transform. Note that this specific time signal was obtained from a $5.43 \mathrm{MHz} 5$-cycle pulse in the calibrated uniform phantom. (Right) Geometry of the $S(x, y)$ frame produced from 256 time signals. Here the x-axis represents the linear array transducer channel number from 0 to 256 , while the $y$-axis is axial depth from the transducer face.

After the generation of $S_{1}(x, y)$ and $S_{2}(x, y)$ frames a crossroad is reached, and the NLSF 
method can either be applied to B-mode imaging or BE imaging for use in noninvasive thermometry.

\section{NLSF B-Mode Imaging}

In order to generate B-mode images using the NLSF method, the Hilbert transform was applied to both $S_{1}(x, y)$ and $S_{2}(x, y)$ and the absolute value taken to find each signals envelope $\left(S_{1_{\text {Envelope }}}(x, y)\right.$ and $S_{2_{\text {Envelope }}}(x, y)$ respectively) as follows:

$$
\begin{aligned}
& S_{1_{\text {Envelope }}}(x, y)=\left|\mathcal{H}\left\{S_{1}(x, y)\right\}\right| \\
& S_{2_{\text {Envelope }}}(x, y)=\left|\mathcal{H}\left\{S_{2}(x, y)\right\}\right|
\end{aligned}
$$

The dynamic range of the two frames was then adjusted by a logarithmic compression function such that:

$$
\begin{aligned}
& L_{1}(x, y)=10^{\left(\frac{-R}{20}\right)}+\left(1-10^{\frac{-R}{20}}\right)\left(\frac{S_{1_{\text {Envelope }}}(x, y)-\min \left[S_{1_{\text {Envelope }}}(x, y)\right]}{\max \left[S_{1_{\text {Envelope }}}(x, y)\right]-\min \left[S_{1_{\text {Envelope }}}(x, y)\right]}\right) \\
& L_{2}(x, y)=10^{\left(\frac{-R}{20}\right)}+\left(1-10^{\frac{-R}{20}}\right)\left(\frac{S_{2_{\text {Envelope }}}(x, y)-\min \left[S_{2_{\text {Envelope }}}(x, y)\right]}{\max \left[S_{2_{\text {Envelope }}}(x, y)\right]-\min \left[S_{2_{\text {Envelope }}}(x, y)\right]}\right)
\end{aligned}
$$

Where $R$ was the range of compression in $d B$, and $\max$ and $\min$ denote the maximum and minimum values of either $S_{1_{\text {Envelope }}}(x, y)$ or $S_{1_{\text {Envelope }}}(x, y)$ respectively. Note that for all B-mode images generated in this work, the value of $R$ was set to $60 \mathrm{~dB}$. Lastly, the following was applied to $L_{1_{d B}}(x, y)$ and $L_{2_{d B}}(x, y)$ to produce B-mode images $\left(B_{1}(x, y)\right.$ and $B_{2}(x, y)$ respectively):

$$
\begin{aligned}
& B_{1}(x, y)=20 \log _{10}\left[L_{1}(x, y)\right] \\
& B_{2}(x, y)=20 \log _{10}\left[L_{2}(x, y)\right]
\end{aligned}
$$

In order to produce a final NLSF B-mode image $\left(B_{\text {Single }}\right)$, both $B_{1}$ and $B_{2}$ were then averaged such that:

$$
B_{\text {Single }}(x, y)=\frac{B_{1}(x, y)+B_{2}(x, y)}{2}
$$

A simplified step-by-step example NLSF method is provided in Figure 2.4. 


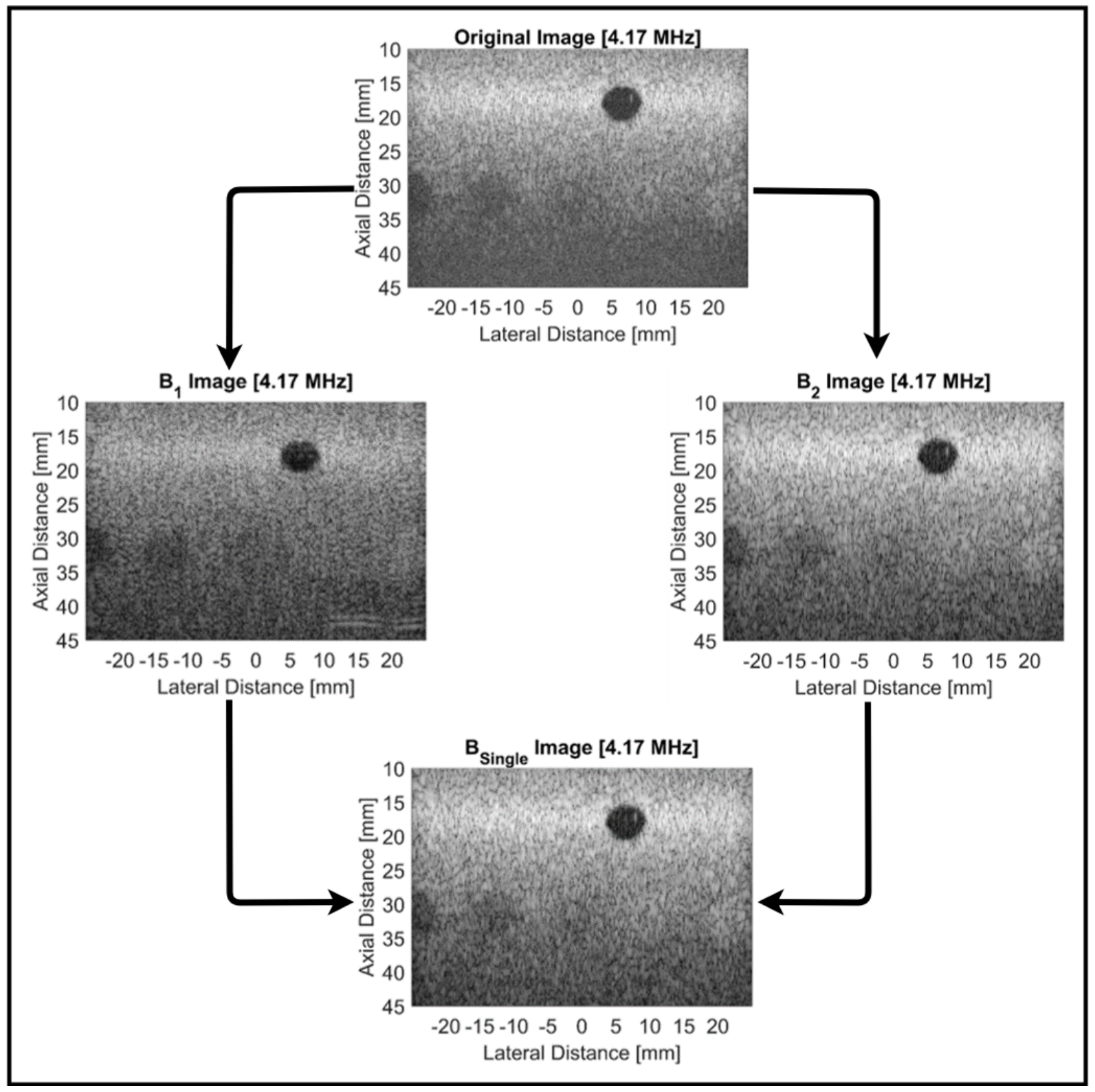

Figure 2.4: A step-by-step diagram of the NLSF method used in the generation of B-mode images. Here a $h_{1}$ and $h_{2}$ image is extracted from an original B-mode image, and then averaged to produce a $B_{\text {Single }}$ image. Note that for this example the original image was obtained using a transmit frequency of 4.17 MHz at a 5-pulse cycle length with the L12-5 linear array transducer in the tissue mimicking phantom. 


\section{NLSF BE Imaging}

In order to generate BE images using the NLSF method, the Hilbert transform was applied to

both $S_{1}(x, y)$ and $S_{2}(x, y)$ and the absolute value taken to find each signals envelope $\left(S_{1_{\text {Envelope }}}(x, y)\right.$ and $S_{2_{\text {Envelope }}}(x, y)$ respectively) as follows:

$$
\begin{aligned}
& S_{1_{\text {Envelope }}}(x, y)=\left|\mathcal{H}\left\{S_{1}(x, y)\right\}\right| \\
& S_{2_{\text {Envelope }}}(x, y)=\left|\mathcal{H}\left\{S_{2}(x, y)\right\}\right|
\end{aligned}
$$

To calculate BE, envelope values were then squared at each data point along each RF line as follows [80, 73]:

$$
\begin{aligned}
& B E_{1}(x, y)=\left[S_{1_{\text {Envelope }}}(x, y)\right]^{2} \\
& B E_{2}(x, y)=\left[S_{2_{\text {Envelope }}}(x, y)\right]^{2}
\end{aligned}
$$

In order to produce a final NLSF BE image ( $\left.B E_{\text {Single }}\right)$, both $B E_{1}$ and $B E_{2}$ were then averaged such that:

$$
B E_{\text {Single }}(x, y)=\frac{B E_{1}(x, y)+B E_{2}(x, y)}{2}
$$

A detailed block diagram outlining both the generation of B-mode and BE images using the NLSF method is provided in Figure 2.5. 


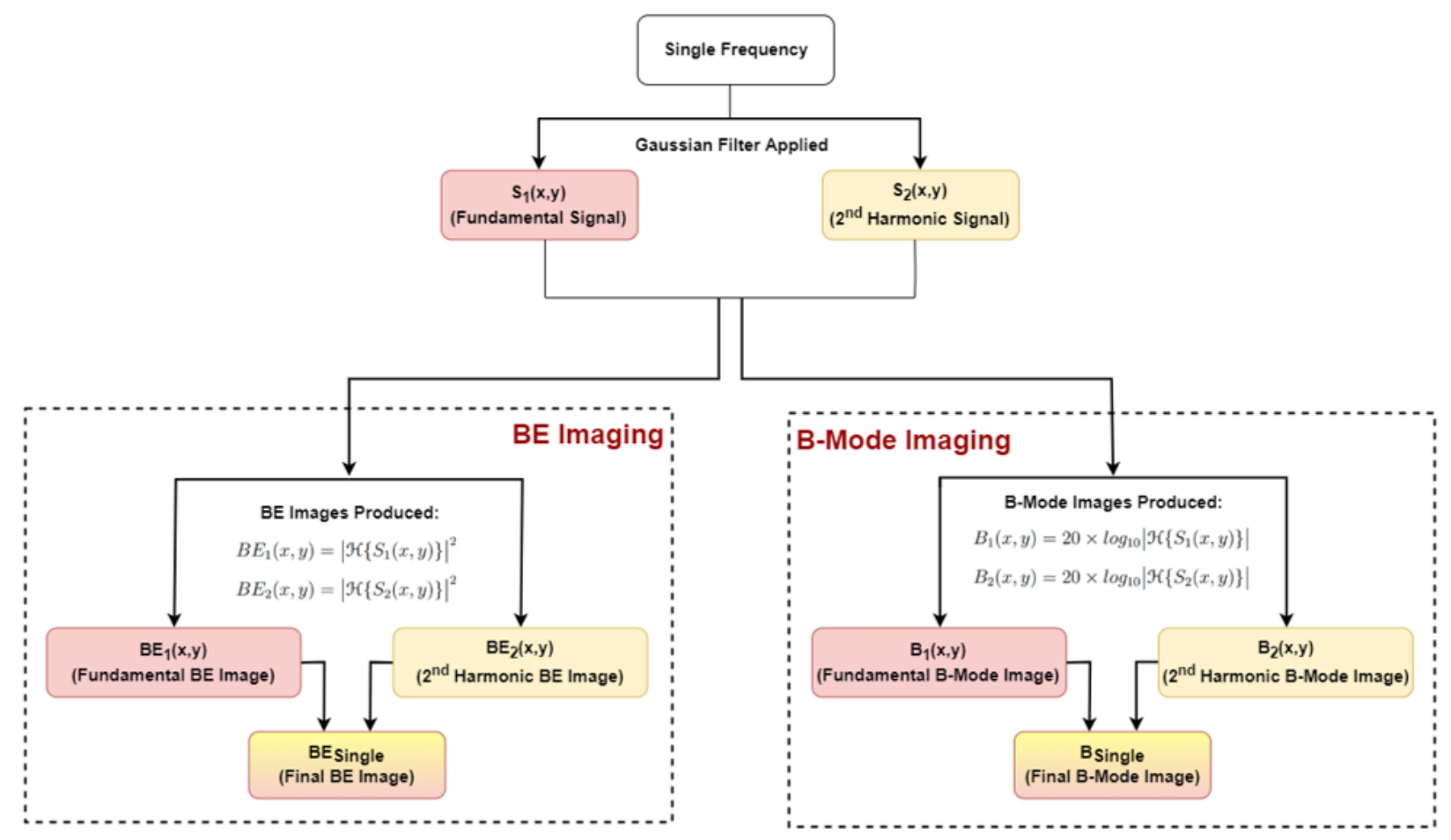

Figure 2.5: Initially a single frame is saved at a single transmit frequency, and Gaussian filters applied to extract $h_{1}$ and $h_{2}$ signals. From this, either B-mode or BE images can be generated.

\subsubsection{NLFC Method}

The NLFC process begins with the transmission of two initial pulses at two different transmit frequencies $(f)$ from the L12-5 (256 channels) linear array transducer, denoted as $p_{f_{1}}$ and $p_{f_{2}}$ respectively. The two received signals, $s_{f_{1}}$ and $s_{f_{2}}$, were then saved as frames 1 and 2 using MATLAB (MathWorks, Natick, MA). A beamforming function and the Fourier transform were then applied to switch to the frequency domain:

$$
\begin{array}{cc}
p_{f_{1}}(t) \underset{\text { Signal received }}{\stackrel{\text { Pulse transmitted }}{\longrightarrow}} s_{f_{1}}(t) \quad, \quad p_{f_{2}}(t) \underset{\text { Signal received }}{\stackrel{\text { Pulse transmitted }}{\longrightarrow}} s_{f_{2}}(t) \\
\mathcal{F}\left\{s_{f_{1}}(t)\right\}=S_{f_{1}}(\omega) \quad, \quad \mathcal{F}\left\{s_{f_{2}}(t)\right\}=S_{f_{2}}(\omega)
\end{array}
$$

To separate the $h_{1}$ and $h_{2}$ signals for each frame, four Gaussian filters were then applied $\left(g_{i, f_{j}}\right)$. Here $j$ is the transmit frequency, while $i$ is 1 for the $h_{1}$ signal and 2 for the $h_{2}$ signal. The inverse Fourier transform was then applied to all $256 \mathrm{RF}$ lines for each signal, generating four $S_{i, f_{j}}(x, y)$ 
frames, as seen in Figure 2.3:

$$
\begin{array}{ll}
S_{1, f_{1}}(x, y)=\mathcal{F}^{-1}\left\{S_{f_{1}}(\omega) \times g_{1, f_{1}}(\omega)\right\} & S_{1, f_{2}}(x, y)=\mathcal{F}^{-1}\left\{S_{f_{2}}(\omega) \times g_{1, f_{2}}(\omega)\right\} \\
S_{2, f_{1}}(x, y)=\mathcal{F}^{-1}\left\{S_{f_{1}}(\omega) \times g_{2, f_{1}}(\omega)\right\} & ,
\end{array}
$$

After the generation of $S_{1, f_{1}}(x, y), S_{1, f_{2}}(x, y), S_{2, f_{1}}(x, y)$, and $S_{2, f_{2}}(x, y)$ frames a crossroad is reached, and the NLFC method can either be applied to B-mode imaging or BE imaging for use in noninvasive thermometry.

\section{NLFC B-Mode Imaging}

In order to generate B-mode images using the NLFC method, the Hilbert transform was applied to $S_{1, f_{1}}(x, y), S_{1, f_{2}}(x, y), S_{2, f_{1}}(x, y)$, and $S_{2, f_{2}}(x, y)$, and the absolute value taken to find each signals envelope $\left(S_{1, f_{1}, \text { Envelope }}(x, y), S_{1, f_{2}, \text { Envelope }}(x, y), S_{2, f_{1}, \text { Envelope }}(x, y)\right.$, and $S_{2, f_{2}, \text { Envelope }}(x, y)$ respectively) as follows:

$$
\begin{array}{ll}
S_{1, f_{1}, \text { Envelope }}(x, y)=\left|\mathcal{H}\left\{S_{1, f_{1}}(x, y)\right\}\right| & , \quad S_{1, f_{2}, \text { Envelope }}(x, y)=\left|\mathcal{H}\left\{S_{1, f_{2}}(x, y)\right\}\right| \\
S_{2, f_{1}, \text { Envelope }}(x, y)=\left|\mathcal{H}\left\{S_{2, f_{1}}(x, y)\right\}\right| & , \quad S_{2, f_{2}, \text { Envelope }}(x, y)=\left|\mathcal{H}\left\{S_{2, f_{2}}(x, y)\right\}\right|
\end{array}
$$

The dynamic range of the four frames was then adjusted by a logarithmic compression function such that:

$$
\begin{aligned}
& L_{1, f_{1}}(x, y)=10^{\left(\frac{-R}{20}\right)}+\left(1-10^{\frac{-R}{20}}\right)\left(\frac{S_{1, f_{1}, \text { Envelope }}(x, y)-\min \left[S_{1, f_{1}, \text { Envelope }}(x, y)\right]}{\max \left[S_{1, f_{1}, \text { Envelope }}(x, y)\right]-\min \left[S_{1, f_{1}, \text { Envelope }}(x, y)\right]}\right) \\
& L_{1, f_{2}}(x, y)=10^{\left(\frac{-R}{20}\right)}+\left(1-10^{\frac{-R}{20}}\right)\left(\frac{S_{1, f_{2} \text { Envelope }}(x, y)-\min \left[S_{1, f_{2} \text { Envelope }}(x, y)\right]}{\max \left[S_{1, f_{2}, \text { Envelope }}(x, y)\right]-\min \left[S_{1, f_{2}, \text { Envelope }}(x, y)\right]}\right)
\end{aligned}
$$




$$
\begin{aligned}
& L_{2, f_{1}}(x, y)=10^{\left(\frac{-R}{20}\right)}+\left(1-10^{\frac{-R}{20}}\right)\left(\frac{S_{2, f_{1}, \text { Envelope }}(x, y)-\min \left[S_{2, f_{1}, \text { Envelope }}(x, y)\right]}{\max \left[S_{2, f_{1}, \text { Envelope }}(x, y)\right]-\min \left[S_{2, f_{1}, \text { Envelope }}(x, y)\right]}\right) \\
& L_{2, f_{2}}(x, y)=10^{\left(\frac{-R}{20}\right)}+\left(1-10^{\frac{-R}{20}}\right)\left(\frac{S_{2, f_{2}, \text { Envelope }}(x, y)-\min \left[S_{2, f_{2}, \text { Envelope }}(x, y)\right]}{\max \left[S_{2, f_{2}, \text { Envelope }}(x, y)\right]-\min \left[S_{2, f_{2}, \text { Envelope }}(x, y)\right]}\right)
\end{aligned}
$$

Where $R$ was the range of compression in $d B$, and $\max$ and min denote the maximum and minimum values of either $S_{1, f_{1}, \text { Envelope }}(x, y), S_{1, f_{2}, \text { Envelope }}(x, y), S_{2, f_{1}, \text { Envelope }}(x, y)$, and $S_{2, f_{2}, \text { Envelope }}(x, y)$ respectively. Note that for all B-mode images generated in this work, the value of $R$ was set to $60 d B$. Lastly, the following was applied to $L_{1, f_{1}, d B}(x, y), L_{1, f_{2}, d B}(x, y), L_{2, f_{1}, d B}(x, y)$, and $L_{2, f_{2}, d B}(x, y)$ to produce B-mode images $\left(B_{1, f_{1}}(x, y), B_{1, f_{2}}(x, y), B_{2, f_{1}}(x, y)\right.$, and $B_{2, f_{2}}(x, y)$ respectively):

$$
\begin{array}{ll}
B_{1, f_{1}}(x, y)=20 \log _{10}\left[L_{1, f_{1}}(x, y)\right] \quad, & B_{1, f_{2}}(x, y)=20 \log _{10}\left[L_{1, f_{2}}(x, y)\right] \\
B_{2, f_{1}}(x, y)=20 \log _{10}\left[L_{2, f_{1}}(x, y)\right] & ,
\end{array}
$$

An average $h_{1}$ and $h_{2}$ B-mode image was then found as follows:

$$
\begin{aligned}
& \overline{B_{1}}(x, y)=\frac{B_{1, f_{1}}(x, y)+B_{1, f_{2}}(x, y)}{2} \\
& \overline{B_{2}}(x, y)=\frac{B_{2, f_{1}}(x, y)+B_{2, f_{2}}(x, y)}{2}
\end{aligned}
$$

Lastly, a final compounded B-mode image, denoted $B_{N L F C}$, was found through averaging $\overline{B_{1}}$ and $\overline{B_{2}}$ :

$$
B_{N L F C}(x, y)=\frac{\overline{B_{1}}(x, y)+\overline{B_{2}}(x, y)}{2}
$$

A step-by-step example of the NLFC method is provided in Figure 2.6. 


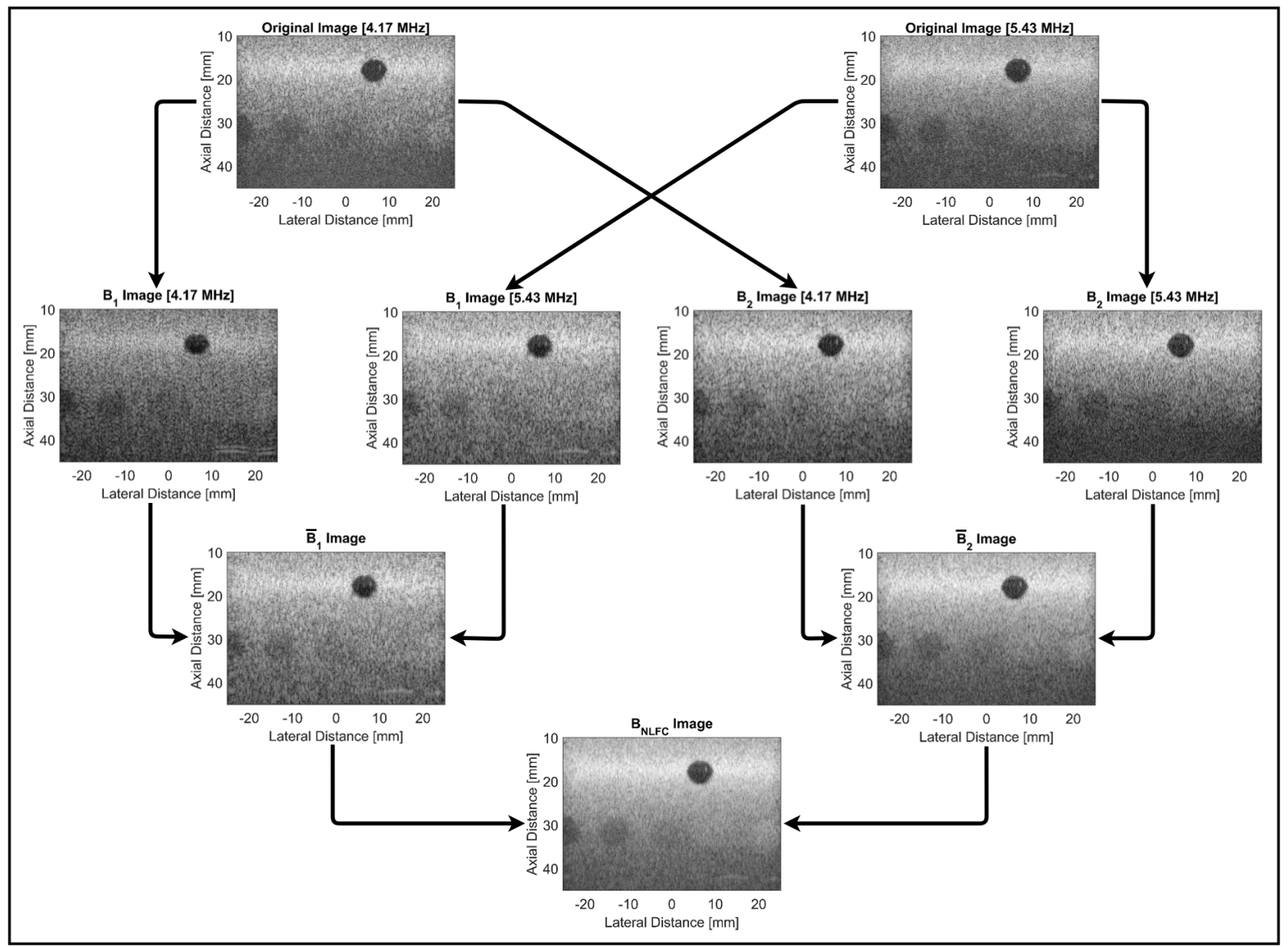

Figure 2.6: A step-by-step diagram of the NLFC method used in the generation of B-mode images. This process was completed using 4.17 MHz and 5.43 MHz frequencies as frame 1 and frame 2 in the compounding process. Both NLSF images are filtered to generate $B_{1}$ and $B_{2}$ images. An average $B_{1}$ and $B_{2}$ image is then found and used to generate a final $B_{N L F C}$ image.

\section{NLFC BE Imaging}

In order to generate BE images using the NLFC method, the Hilbert transform was applied to $S_{1, f_{1}}(x, y), S_{1, f_{2}}(x, y), S_{2, f_{1}}(x, y)$, and $S_{2, f_{2}}(x, y)$, and the absolute value taken to find each signals envelope $\left(S_{1, f_{1}, \text { Envelope }}(x, y), S_{1, f_{2}, \text { Envelope }}(x, y), S_{2, f_{1}, \text { Envelope }}(x, y)\right.$, and $S_{2, f_{2}, \text { Envelope }}(x, y)$ respectively) as follows:

$$
\begin{aligned}
& S_{1, f_{1}, \text { Envelope }}(x, y)=\left|\mathcal{H}\left\{S_{1, f_{1}}(x, y)\right\}\right| \quad, \quad S_{1, f_{2}, \text { Envelope }}(x, y)=\left|\mathcal{H}\left\{S_{1, f_{2}}(x, y)\right\}\right| \\
& S_{2, f_{1}, \text { Envelope }}(x, y)=\left|\mathcal{H}\left\{S_{2, f_{1}}(x, y)\right\}\right| \quad, \quad S_{2, f_{2}, \text { Envelope }}(x, y)=\left|\mathcal{H}\left\{S_{2, f_{2}}(x, y)\right\}\right|
\end{aligned}
$$


To calculate BE, envelope values were then squared at each data point along each RF line as follows [80, 73]:

$$
\begin{array}{ll}
B E_{1, f_{1}}(x, y)=\left[S_{1, f_{1}, \text { Envelope }}(x, y)\right]^{2} & \quad B E_{1, f_{2}}(x, y)=\left[S_{1, f_{2}, \text { Envelope }}(x, y)\right]^{2} \\
B E_{2, f_{1}}(x, y)=\left[S_{2, f_{1}, \text { Envelope }}(x, y)\right]^{2} & ,
\end{array}
$$

An average $h_{1}$ and $h_{2}$ BE image was then found as follows:

$$
\begin{aligned}
& \overline{B E_{1}}(x, y)=\frac{B E_{1, f_{1}}(x, y)+B E_{1, f_{2}}(x, y)}{2} \\
& \overline{B E_{2}}(x, y)=\frac{B E_{2, f_{1}}(x, y)+B E_{2, f_{2}}(x, y)}{2}
\end{aligned}
$$

Lastly, a final compounded $\mathrm{BE}$ image, denoted $B E_{N L F C}$, was found through averaging $\overline{B E_{1}}$ and $\overline{B E_{2}}$ :

$$
B E_{N L F C}(x, y)=\frac{\overline{B E_{1}}(x, y)+\overline{B E_{2}}(x, y)}{2}
$$

A detailed block diagram outlining both the generation of B-mode and BE images using the NLFC method is provided in Figure 2.7. 


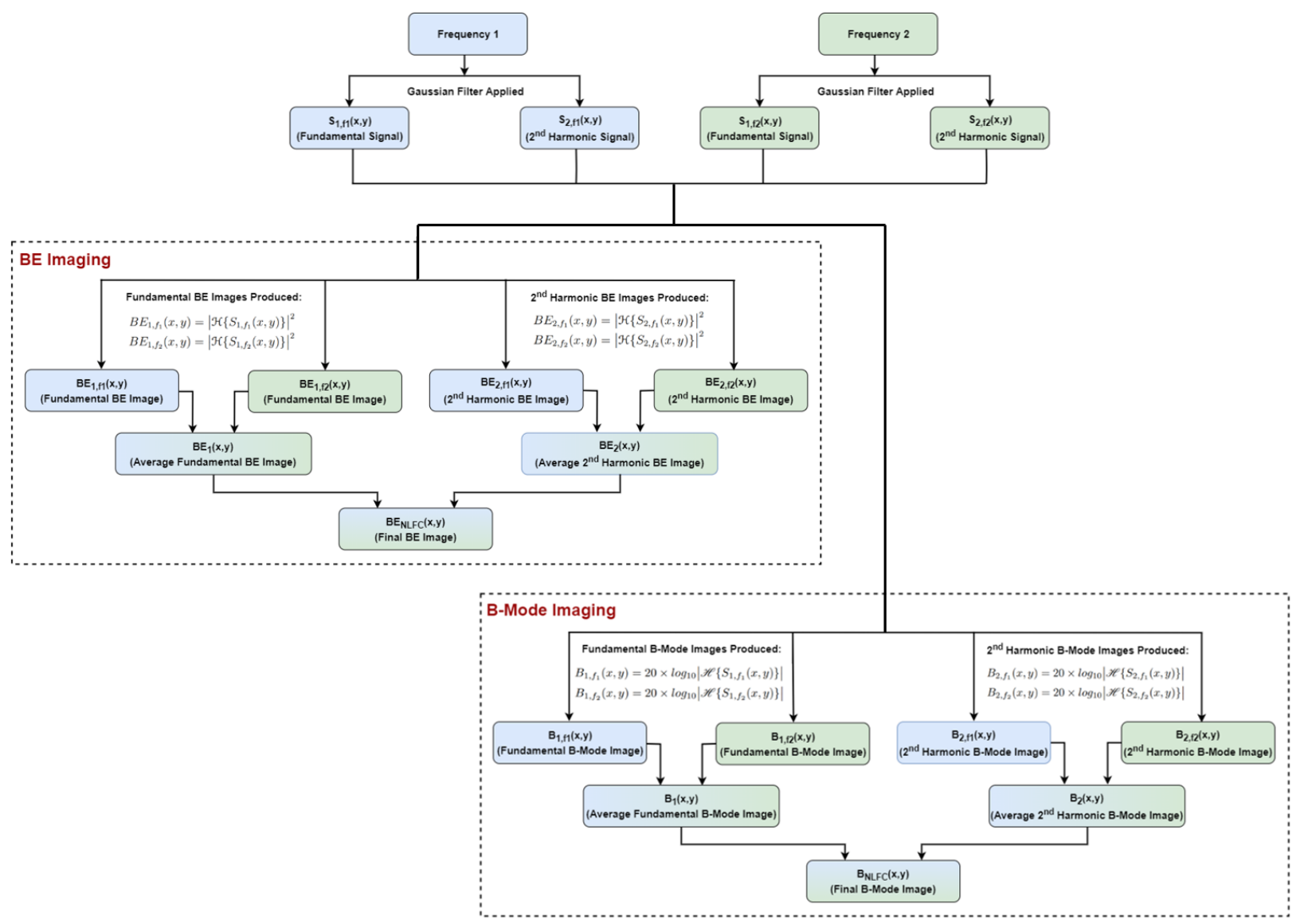

Figure 2.7: Initially two frames are saved at two different transmit frequencies, and Gaussian filters applied to extract $h_{1}$ and $h_{2}$ signals for each frequency. From this, either B-mode or BE images can be generated.

\subsubsection{Determining NLFC Input Parameters}

In order to optimize the NLFC Matlab code, three input parameters were selected to maximize SNR in both B-mode and BE images. These input parameters included the center frequencies of the first and second transmitted pulse, saved as frames 1 and 2 respectively, as well as the number of cycles per pulse.

\section{Determining Center Frequencies}

To determine the two center frequencies for use with the NLFC method, both $B_{\text {Single }}$ and $B E_{\text {Single }}$ images of the calibrated TMP were generated using the L12-5 imaging transducer for all center frequencies allowed by the Verasonics system in the range of $3 \mathrm{MHz}$ to $12.5 \mathrm{MHz}$ and a 
fixed 4 cycle pulse length. This range of frequencies was selected to ensure that both the $h_{1}$ and $h_{2}$ component of the signal would fall within the L12-5 transducer bandwidth, as shown in Figure 2.1. The experimental set up used for all TMP measurements, as well as a schematic of the TMP geometry is depicted in Figure 2.8.
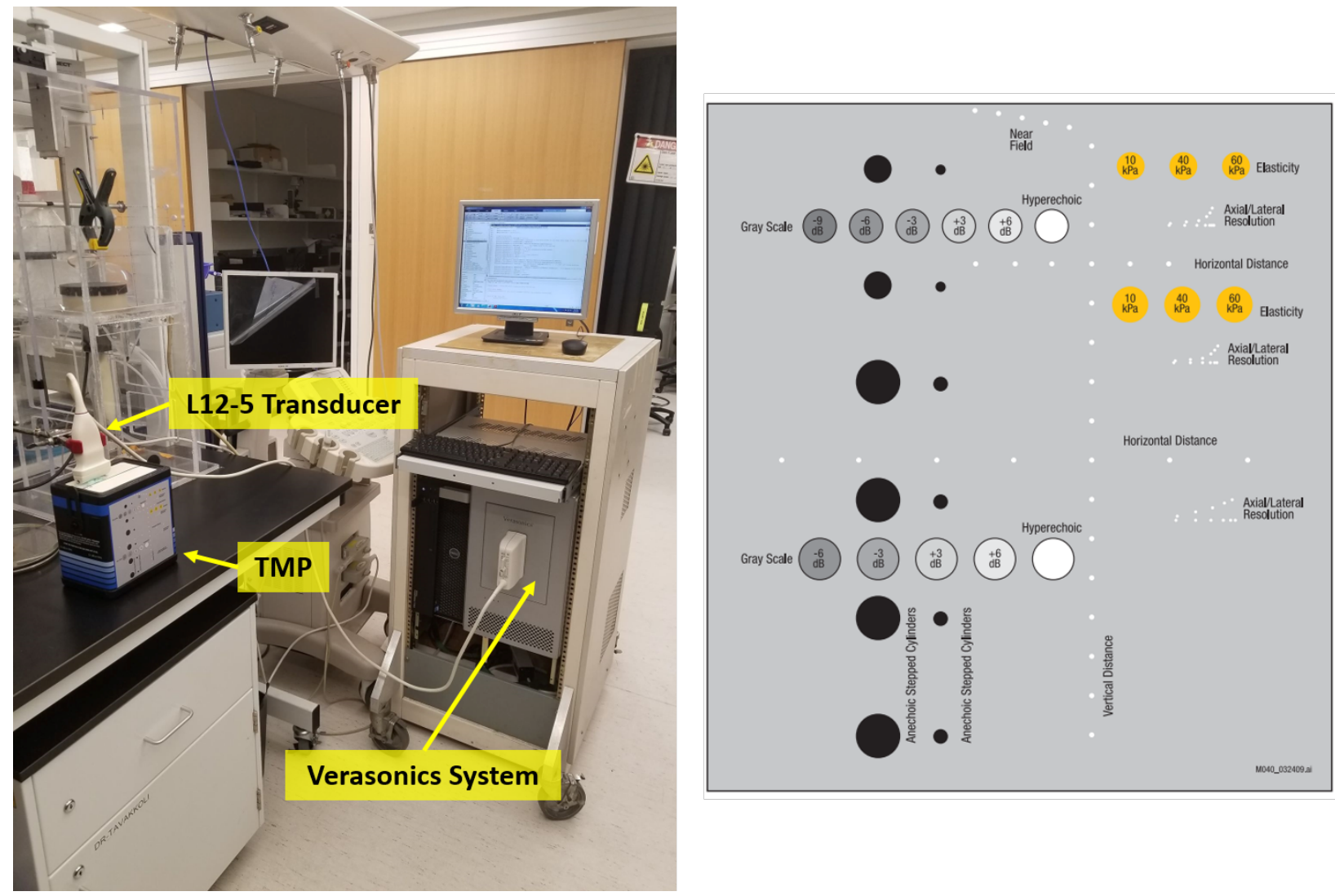

Figure 2.8: (Left) The experimental setup used for determining NLFC input parameters. (Right) a schematic of the calibrated TMP used for all measurements.

For all $B_{\text {Single }}$ and $B E_{\text {Single }}$ images SSNR was then calculated in a uniform region at the focal depth of the L12-5 imaging transducer as follows [13, 14]:

$$
S S N R=\frac{\mu_{S}}{\sigma_{S}}
$$

Here $\mu_{S}$ is the average pixel intensity in the uniform region, while $\sigma_{S}$ is the standard deviation in pixel intensity in the same region. For all images a hypoechoic cyst was imaged in the TMP at the focal depth, and the uniform region was defined as a rectangular area on either side of the cyst. 
$\mu_{S}$ and $\sigma_{S}$ were then taken as the mean and standard deviation of pixel intensity inside the two marked regions. An example of a typical $B_{\text {Signal }}$ B-mode image is shown in Figure 2.9 with the two uniform regions marked in yellow.

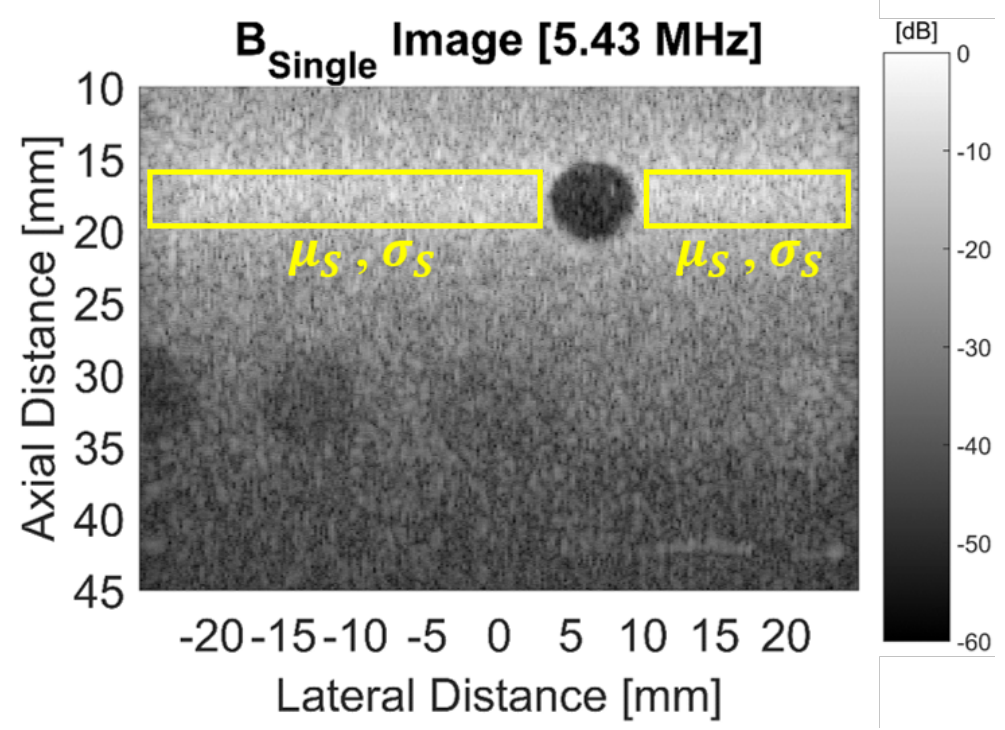

Figure 2.9: A typical $B_{\text {Single }}$ image produced with a $5.43 \mathrm{MHz}$ transmit frequency at a 4 cycle pulse length, The uniform regions used for SSNR calculation are marked as yellow rectangles. Here $\mu_{S}$ and $\sigma_{S}$ are taken as the mean and standard deviation respectively of pixel values inside of the marked uniform regions.

By plotting $B_{\text {Single }}$ and $B E_{\text {Single }}$ SSNR as a function of center frequency, a peak in SSNR was then identified for both cases. To determine optimal input center frequencies for the NLFC method, all possible frequency pairs in the SSNR peak were then input into the NLFC code, and SSNR of the produced $B_{N L F C}$ and $B E_{N L F C}$ images calculated. The frequency pair which maximized both $B_{N L F C}$ and $B E_{N L F C}$ SSNR was then selected as the NLFC center frequencies.

\section{Determining Number of Cycles of Excitation Pulse}

When considering the number of cycles of excitation pulse $(N)$ to use in the NLFC method, there is a trade-off between SSNR and axial resolution. As the number of cycles is increased the SSNR of the produced B-mode or BE image will also increase, however there is a subsequent loss of axial resolution [83]. Although the objective of the NLFC method was to maximize SSNR, an axial resolution limit $(A R L)$ was also considered to ensure the method could be applied to thermometry 
applications (see section 2.2). For this purpose, the $A R L$ for the NLFC method was defined as the diameter of a $1 \mathrm{MHz} 125 \mathrm{~mm}$ diameter high power transducer (Imasonic, Voray-sur-l'Ognon, France) which would be used to perform tissue heating in future experiments. To determine the focal point diameter of the LIFU transducer in question, and therefore the $A R L$ of the NLFC method, a full 3D multi-layer COMSOL based simulation model (COMSOL Multiphysics Modeling Software, Stockholm, Sweden) was used to simulate the LIFU heating experiment outlined in section 2.2.3. The model used was capable of simulating 3D multi-layer medium geometries and could generate realistic 3D intensity and tissue temperature profiles [84]. A value for $A R L$ was then determined as the full width half maximum (FWHM) of the focal point lateral intensity profile.

In order to calculate the required number of cycles from the $A R L$ the following definition of spatial pulse length $(S P L)$ was used:

$$
\begin{gathered}
S P L=2 \times A R L \\
S P L=N \times \lambda=N \times\left(\frac{c}{f}\right)
\end{gathered}
$$

Note that here $\lambda$ is the wavelength of the center frequency $(f)$ of the transmitted ultrasound wave, while $c$ is the SOS in soft tissue. From Equations 2.22 and 2.23, a value for $N$ was calculated for both NLFC input frequencies as follows:

$$
N=2 \times A R L \times\left(\frac{f}{c}\right)
$$

The maximum value of $N$ at which the $A R L$ is satisfied was then selected for use with the NLFC method. Using the optimized input parameters the B-mode images were then generated with the NLFC method and the percent improvement in SSNR over the previous NLSF method was quantified. 


\subsubsection{Quantifying The Improvement In B-Mode and BE Image SSNR Due To The NLFC Method}

In order to quantify the improvement in B-mode image SSNR as a result of the NLFC method, SSNR was calculated for $B_{N L F C}, B_{\text {Single }}(4.17 \mathrm{MHz})$ and $B_{\text {Single }}(5.43 \mathrm{MHz})$ images over six

trials. Similarly, SSNR of BE images was calculated for $B E_{N L F C}, B E_{\text {Single }}(4.17 \mathrm{MHz})$ and $B E_{\text {Single }}(5.43 \mathrm{MHz})$ over six trials. For all 6 trials the percent improvement in SSNR as a result of the NLFC method was then calculated as follows:

$$
\begin{aligned}
& \text { B-Mode Percent Improvement }(\%)=\frac{B_{N L F C}-B_{\text {Single }}}{B_{\text {Single }}} \times 100 \% \\
& \text { BE Percent Improvement }(\%)=\frac{B E_{N L F C}-B E_{\text {Single }}}{B E_{\text {Single }}} \times 100 \%
\end{aligned}
$$

By averaging calculated SSNR and percent improvement over all six trials, the improvements in B-mode and BE image SSNR due to the NLFC method were quantified.

\subsection{Development and Application of The NLFC Method In Non- linear Ultrasound Thermometry}

In this section a clinically relevant heating modality was introduced in the form of a therapeutic HIFU transducer. With this form of tissue heating, localized heating of a small area can be achieved within the target tissue. Using a modified version of both the previous NLSF and the NLFC methods introduced in section 2.1, 2D maps of $h \mathrm{CBE}$ were generated for localized LIFU heating of ex vivo porcine muscle tissue. A comparative study was then performed to quantify percent improvements in SNR of 2D $h \mathrm{CBE}$ maps as a result of the NLFC method. Lastly, $h$ CBE-to-temperature calibration was performed using both the NLFC and NLSF methods, and 2D maps of temperature generated. Lastly, both the NLSF and NLFC temperature maps were compared with a theoretical COMSOL based model. 


\subsubsection{NLSF Method $h$ CBE Calculation}

After calculating $B E_{\text {Single }}(x, y)$ for every timestamp $(\tau), h \mathrm{CBE}$ can be calculated by comparing to a baseline measurement $(\tau=0)$ at a known reference temperature. In order to stabilize the division when calculating $h \mathrm{CBE}$, a stabilizing constant, $D$, was also added to the denominator such that:

$$
h \mathrm{CBE}(x, y, \tau)=\left[\frac{B E_{\text {Single }}(x, y, \tau)-B E_{\text {Single }}(x, y, 0)}{B E_{\text {Single }}(x, y, 0)+D}\right] \times 100
$$

Here $D$ is arbitrarily defined as $1 \%$ of the maximum (Max) value of the $B E_{\text {Single }}$ baseline image as follows:

$$
D=\operatorname{Max}\left[B E_{\text {Single }}(x, y, 0)\right] \times(0.01)
$$

From this, $h \operatorname{CBE}(x, y, \tau)$ can be plotted as a $2 \mathrm{D}$ colormap representing change in backscattered energy of acoustic harmonics as a ROI is heated.

\subsubsection{NLFC Method $h$ CBE Calculation}

After calculating $B E_{N L F C}(x, y)$ for every timestamp $(\tau), h \mathrm{CBE}$ can be calculated in two steps. First, an intermediate step was used to calculate harmonic CBE between each consecutive time stamp as $h \mathrm{CBE}^{\prime}$. Note that in order to stabilize the division when calculating $h \mathrm{CBE}^{\prime}$, a stabilizing constant, $D$, was also added to the denominator as follows:

$$
h \operatorname{CBE}^{\prime}(x, y, \tau)=\left[\frac{B E_{N L F C}(x, y, \tau)-B E_{N L F C}(x, y, \tau-1)}{B E_{N L F C}(x, y, \tau-1)+D}\right] \times 100
$$

Here $D$ is arbitrarily defined as $1 \%$ of the maximum (Max) value of the $B E_{N L F C}(x, y, 0)$ image as follows:

$$
D=\operatorname{Max}\left[B E_{N L F C}(x, y, 0)\right] \times(0.01)
$$

To calculate a final value for $h \mathrm{CBE}$ at each time stamp, a cumulative sum was then applied to sum all minute-to-minute $h \mathrm{CBE}^{\prime}$ values up to the current time stamp, $\tau$, such that:

$$
h \mathrm{CBE}(x, y, \tau)=\sum_{k=1}^{\tau}\left[h \operatorname{CBE}^{\prime}(x, y, k)\right]
$$


This cumulative sum method of calculating $h \mathrm{CBE}$ at each time stamp has been used in MR thermometry previously to account for measurement-to-measurement motion, and was utilized in this work to minimize motion artifacts from sample motion over the measurement period [85]. A block diagram of the modified NLSF and NLFC methods is provided in Figure 2.10.

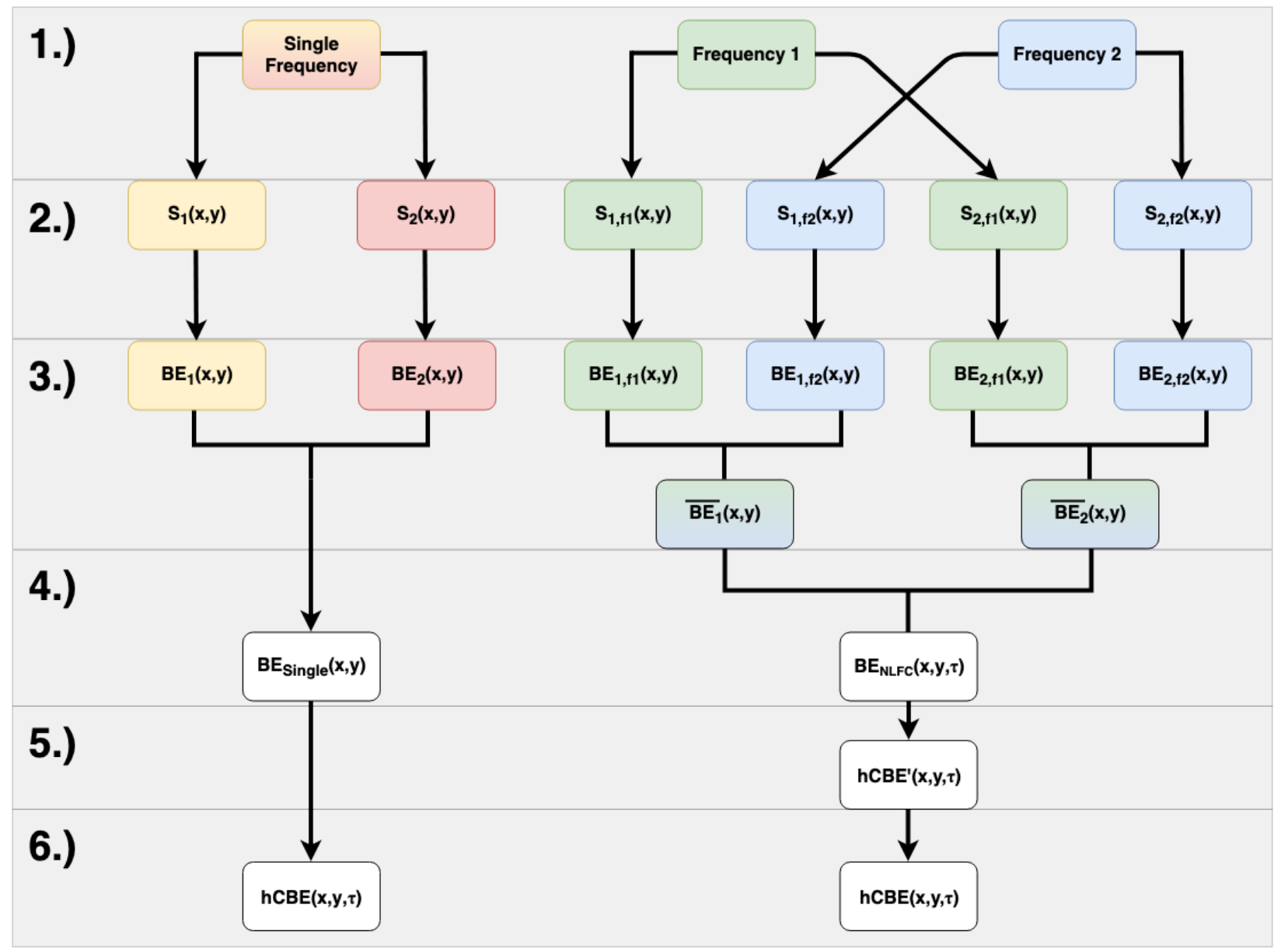

Figure 2.10: The two methods are broken down into 6 steps such that: (1) In the NLSF method one frame at a single transmit frequency is saved. In the NLFC method two frames are saved at two different transmit frequencies. Gaussian filters are then applied to extract the $H_{1}$ and $h_{2}$ signals. (2) $S(x, y)$ frames are formed for $h_{1}$ and $h_{2}$ of each frequency (3) $B E$ calculated from each $S(x, y)$ frame. In the NLFC method an extra step is included to find average backscattered energy for both $h_{1}$ and $h_{2}$ signals. (4) Two backscattered energy images are averaged to produce a final backscattered energy image in both methods. (5) An intermediate step is included in the NLFC method only, to find frame-to-frame difference in $h \mathrm{CBE}$. (6) $h \mathrm{CBE}$ is found compared to a baseline measurement in the NLSF method, while a cumulative sum is performed in the NLFC method.

In order to further reduce the generation of motion artifacts, two additional features were also 
added to the NLFC method. The first, a motion compensation function, was added to correct for motion between consecutive measurements. Motion compensation was performed in both the axial and lateral directions using cross correlation between consecutive $B E_{N L F C}$ images. Here a correlation coefficient was calculated for each consecutive frame and a correction factor applied in both the lateral and axial direction to account for tissue motion. The second feature, a gradient filter, was applied to correct for rotational motion near the edges of the $h \mathrm{CBE}$ images. This operated by applying a decreasing gradient factor to all $h \mathrm{CBE}$ values as a function of radius from the therapeutic transducer focal point. From this, $h \mathrm{CBE}(x, y, \tau)$ can be plotted as a $2 \mathrm{D}$ colormap representing change in backscattered energy of acoustic harmonics in a ROI during heating.

\subsubsection{LIFU Heating of Ex Vivo Porcine Muscle Tissue}

A single channel function generator (AFG3101 Arbitrary Function generator, Tektronix Inc., Beaverton, Oregon, United States) and AG series amplifier (T \& C Power, Rochester, New York, United States) were connected to a $1 \mathrm{MHz} 125 \mathrm{~mm}$ diameter high power transducer (Imasonic, Voray-sur-l'Ognon, France) in series. The high power transducer was kept fixed to the side of a

plexiglass water tank with an oppositely placed acoustic absorber to prevent reflection. All heating was performed with both the high power transducer and sample submerged in purified water. To measure BE of the $h_{1}$ and $h_{2}$ signals the L12-5 imaging transducer paired with a Verasonics ${ }^{\circledR}$ ultrasound research system, was used to transmit pulse trains of 5-cycle length at transmit frequency of alternating 4.17 MHz and 5.43 MHz. Note that these input parameters were selected based on tests of the NLFC method in B-mode and BE imaging of a calibrated uniform phantom (see section 2.1). Due to the bandwidth of the L12-5 imaging transducer, it was not feasible to extract the third harmonic signal as done in the previous work [73].

To prepare the LIFU heating samples, freshly excised ex vivo porcine muscle tissue bought from a local deli was submerged in purified water for 24 hours prior to each experiment to remove air bubbles. The ex vivo tissue sample was then placed in a 3D printed plastic sample holder within the plexiglass water tank and kept submerged in purified water for the entire experiment. The sample holder was composed of hard plastic with an ultrasound-transparent film normal to the high power transducer. Throughout all experiments a water heater (Haake DC 10 Thermo Controller 
003-2859, ThermoFisher Scientific, Waltham, Massachusetts, United States) was used to keep the tank temperature fixed at $37^{\circ} \mathrm{C}$ to simulate clinically relevant background temperature, and an Omega $^{\mathrm{TM}}$ thermometer (HH309A Four-Channel Data Logger, Omega Engineering, Norwalk, Connecticut, United States) was used to monitor temperature both at the face of the high power transducer and the sample. Note that all water used in the LIFU heating experiments was purified using a Millipore UV ultrapure water purification system (Milli-Q ${ }^{\circledR}$ Integral water purification system, Sigma-Aldrich, St. Louis., Missouri, United States). An overview of the experimental set up is shown in Figure 2.11.

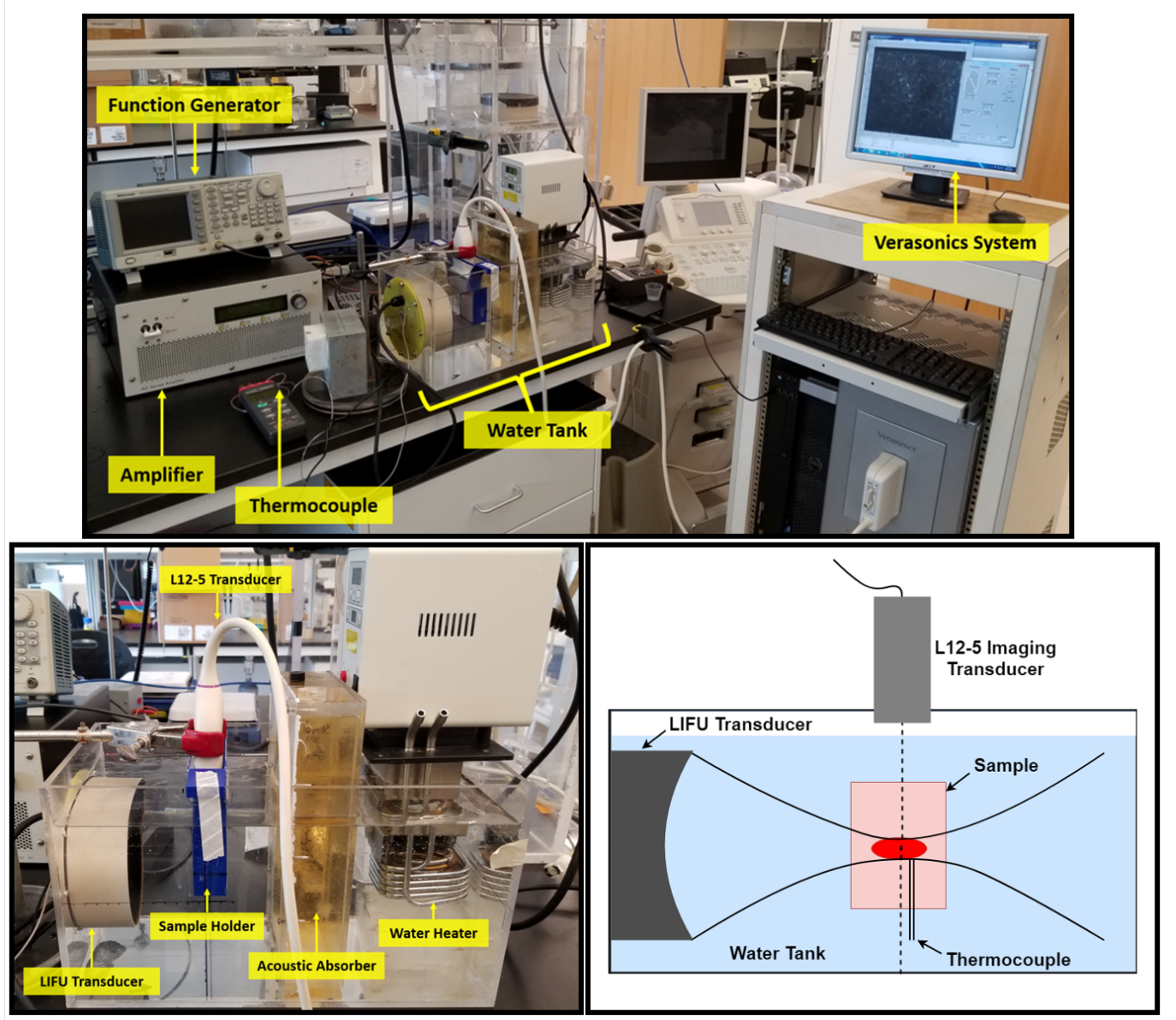

Figure 2.11: The experimental setup used for LIFU heating of ex vivo porcine muscle tissue. (Top) An overview of all major components. (Bottom Left) A close up of the plexiglass water tank. (Bottom Right) The beam overlap geometry of the LIFU transducer and L12-5 imaging transducer. Here the heated region in the sample is depicted as a red circle of $1.1 \mathrm{~mm}$ diameter. The imaging plane of the L12-5 transducer is shown as a dashed line. For the purposes of thermometry calibrations a thermocouple can also be inserted into the heated region.

To perform heating of the ex vivo tissue sample the function generator was used to generate 
a $175 \mathrm{mVpp}, 1 \mathrm{MHz}, 1000$ Cycle, 2 ms trigger interval (50\% dead time) signal for a six minute heating time. With these settings the high power transducer is considered to be running at low power (LIFU regime). After six minutes of heating the LIFU transducer was turned off and the sample was allowed to cool in the $37^{\circ} \mathrm{C}$ water bath for thirty minutes. Every minute for the duration of the thirty six minute experiment the therapeutic transducer was switched off and a measurement was performed with the orthogonally placed L12-5 imaging transducer. Two frames at transmit frequencies of 4.17 MHz and 5.43 MHz were saved for each measurement and input into both the NLFC and NLSF methods for comparison.

\subsection{4 hCBE-to-Temperature Calibration}

In order to estimate temperature at the focal point of the LIFU transducer, the LIFU heating experiment was repeated twelve times in total. For six trials a thermocouple was inserted at the LIFU transducer focal point, and temperature measurements performed every minute for the entire 36 minute experiment using a digital thermometer (Omegaette HH306, Omega Engineering Inc., Stamford, CT). For the other six trials the standard procedure was followed with no thermocouple inserted. In the produced $h \mathrm{CBE}$ maps from these six trials the mean $h \mathrm{CBE}$ value was calculated within a circular ROI of $1 \mathrm{~mm}$ diameter centered at the LIFU transducer focal point. The assumption was made that the average $h \mathrm{CBE}$ value found in this $\mathrm{ROI}$ is obtained at the same position as the thermocouple measurements. By plotting the average focal point $h \mathrm{CBE}$ against thermocouple temperature measurements a calibration curve was then generated using a quadratic curve fit, with the equation of the fit taken as a $h \mathrm{CBE}$-to-temperature calibration function. This conversion function was then applied to every data point in the $h \mathrm{CBE}$ maps To generate $2 \mathrm{D}$ maps of temperature.

\subsubsection{COMSOL Simulation of 2D Temperature Maps}

After producing 2D temperature maps for both the NLSF and NLFC methods, the LIFU heating experiment was simulated using a theoretical COMSOL based model. Here a COMSOL Multiphysics with the bio-heat transfer module (COMSOL Multiphysics Modeling Software,

Stockholm, Sweden) was utilized to generate 2D maps of temperature for localized heating of 
ex vivo porcine tissue with similar heating parameters. The full 3D multi-layer COMSOL based simulation model used was capable of simulating 3D multi-layer medium geometries and could generate realistic 3D intensity and tissue temperature profiles [84]. Important thermal and acoustic parameters used in the simulation are provided in Table 2.1. A schematic of the simulated 3D multi-layer geometry is also provided in Figure 2.12. The simulated 2D temperature maps were then compared with the experimentally produced NLSF and NLFC 2D temperature maps and the agreement quantified.

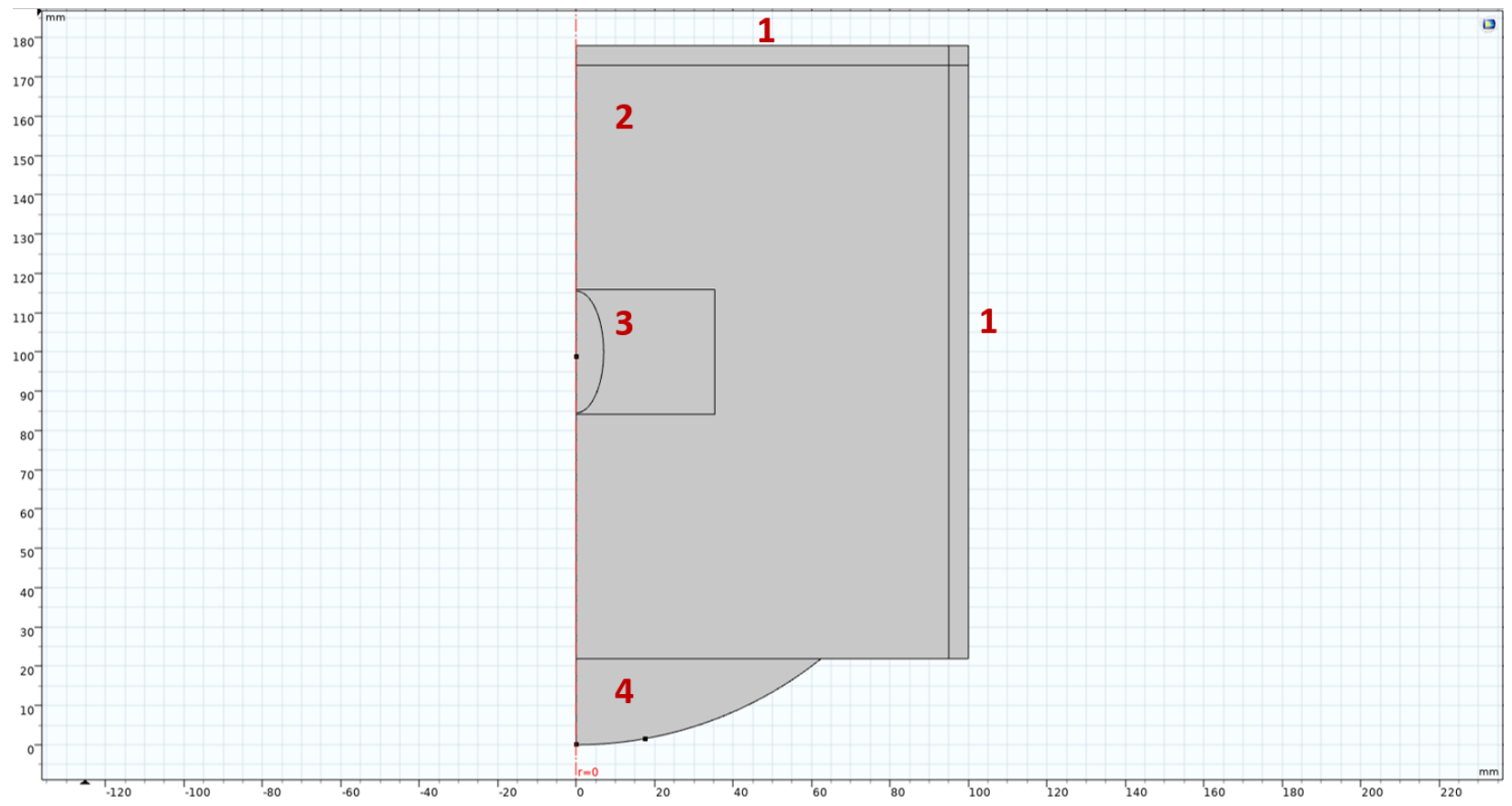

Figure 2.12: The 3D multi-layer geometry simulated using COMSOL. Perfectly matched layers used to simulate the edges of the water tank are labelled as (1). Here two materials are used with water domains marked as (2) and porcine tissue as (3), while the LIFU transducer is labelled as (4). Since the experimental geometry is symmetrical, only half the tank is simulated and then mirrored to reduce the required computational time. Note that an additional spherical domain is included at the LIFU focal point with a smaller element size to improve accuracy. In all domains a maximum element size of $1 / 4$ the LIFU transducer wavelength is used, while in the LIFU focal point spherical domain a maximum element size of $1 / 6$ the wavelength is used. 


\begin{tabular}{ccc}
\hline \hline Parameter & Value & Unit \\
\hline Acoustic Output Power & 6.80 & $\mathrm{~W}$ \\
Attenuation Coefficient of Porcine Tissue $\left(\alpha_{\text {Tissue }}\right)$ & 0.80 & $\mathrm{~dB} / \mathrm{cm}$ \\
Attenuation Coefficient of Water $\left(\alpha_{W}\right)$ & 0.0022 & $\mathrm{~dB} / \mathrm{cm}$ \\
Heat Capacity at Constant Pressure of Porcine Tissue $\left(C_{P, T i s s u e}\right)$ & 3720.00 & $\mathrm{~J} / \mathrm{kg} \cdot \mathrm{K}$ \\
Heat Capacity at Constant Pressure of Water $\left(C_{P, W}\right)$ & 4178.60 & $\mathrm{~J} / \mathrm{kg} \cdot \mathrm{K}$ \\
Density of Porcine Tissue $\left(\rho_{\text {Tissue }}\right)$ & 1091.00 & $\mathrm{~kg} / \mathrm{m}^{3}$ \\
Density of Water $\left(\rho_{W}\right)$ & 993.25 & $\mathrm{~kg} / \mathrm{m}^{3}$ \\
Thermal Conductivity of Porcine Tissue $\left(k_{\text {Tissue }}\right)$ & 0.46 & $\mathrm{~W} / \mathrm{m} \cdot \mathrm{K}$ \\
Thermal Conductivity of Water $\left(k_{W}\right)$ & 0.62 & $\mathrm{~W} / \mathrm{m} \cdot \mathrm{K}$ \\
Speed of Sound of Porcine Tissue $\left(c_{\text {Tissue }}\right)$ & 1593.00 & $\mathrm{~m} / \mathrm{s}$ \\
Speed of Sound of Water $\left(c_{\text {Tissue }}\right)$ & 1520.58 & $\mathrm{~m} / \mathrm{s}$ \\
\hline
\end{tabular}

Table 2.1: Important thermal and acoustic parameters used in the COMSOL simulation [86, 87].

\subsubsection{Quantifying SNR Improvement With The NLFC Method}

In order to quantify improvements in $2 \mathrm{D} h \mathrm{CBE}$ map image quality as a result of the NLFC method, SNR was calculated for both NLFC and NLSF $h$ CBE maps over six trials and compared. Since there is currently no established method for calculating $2 \mathrm{D} h \mathrm{CBE}$ map SNR, a modified B-mode image SNR formula was used [88, 89]. The percent improvement in image SNR as a result of the NLFC method was then calculated and averaged over all six trials:

$$
\begin{gathered}
S N R=\frac{\mu_{S}}{\sigma_{S}} \\
\text { Percent Improvement }(\%)=\frac{h C B E(N L F C)-h C B E(N L S F)}{h C B E(N L S F)} \times 100 \%
\end{gathered}
$$

Here $\mu_{S}$ was taken as the mean pixel intensity in a $1 \mathrm{~mm}$ diameter ROI centered at the LIFU transducer focal point. This ROI was selected to ensure that an area of uniform signal was used for all images tested. $\sigma_{S}$ was taken as the standard deviation of pixel intensity in a uniform region outside of the heated area. This area was selected at a sufficient radius from the LIFU focal point such that any signal inside was assumed to be noise. A typical example of $\mu_{S}$ and $\sigma_{S}$ identification is provided in Figure 2.13. For all six trials used in the $h \mathrm{CBE}$-to-temperature calibration experiments SNR was calculated in both NLFC and NLSF heating phase $h$ CBE maps and compared. 


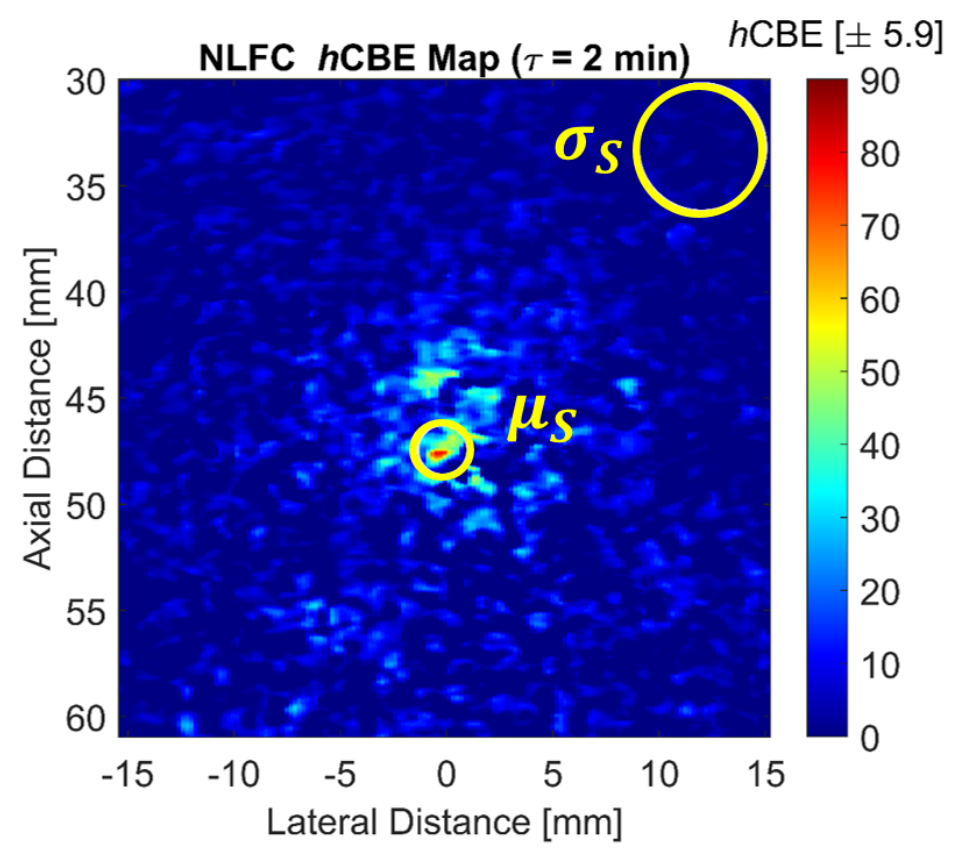

Figure 2.13: A 2D $h \mathrm{CBE}$ map produced using the NLFC for localized LIFU heating of an ex vivo porcine muscle tissue sample. To calculate SNR, $\mu_{S}$ and $\sigma_{S}$ are taken as the mean and standard deviation of pixel intensity inside of manually placed ROIs.

Lastly, experimental temperature profiles of the NLSF and NLFC temperature maps were compared with the COMSOL simulated temperature maps. The lateral temperature profiles were found at the LIFU focal point depth for all simulated, NLFC, and NLSF temperature maps. For both NLFC and NLSF temperature maps the lateral temperature profile was taken by averaging four line profiles at different angles through the LIFU transducer focal point, as seen in Figure 2.14. The root mean squared error (RMSE) between simulation and experimental temperature profiles for each time stamp was then calculated for each NLSF and NLFC experimental image as follows:

$$
R M S E=\sqrt{\text { mean }\left([\text { Simulation }- \text { Experiment }]^{2}\right)}
$$

Calculated RMSE values for the NLFC method ( $R M S E_{N L F C}$ ), and NLSF method using 4.17 $\mathrm{MHz}\left(R M S E_{\text {Single }}(4.17 \mathrm{MHz})\right)$ and $5.43 \mathrm{MHz}\left(R M S E_{\text {Single }}(5.43 \mathrm{MHz})\right)$ transmit frequencies were then compared and the percent improvement as a result of the NLFC method calculated as follows:

$$
\text { Percent Improvement }(\%)=\frac{\left|R M S E_{N L F C}-R M S E_{\text {Single }}\right|}{R M S E_{\text {Single }}} \times 100 \%
$$




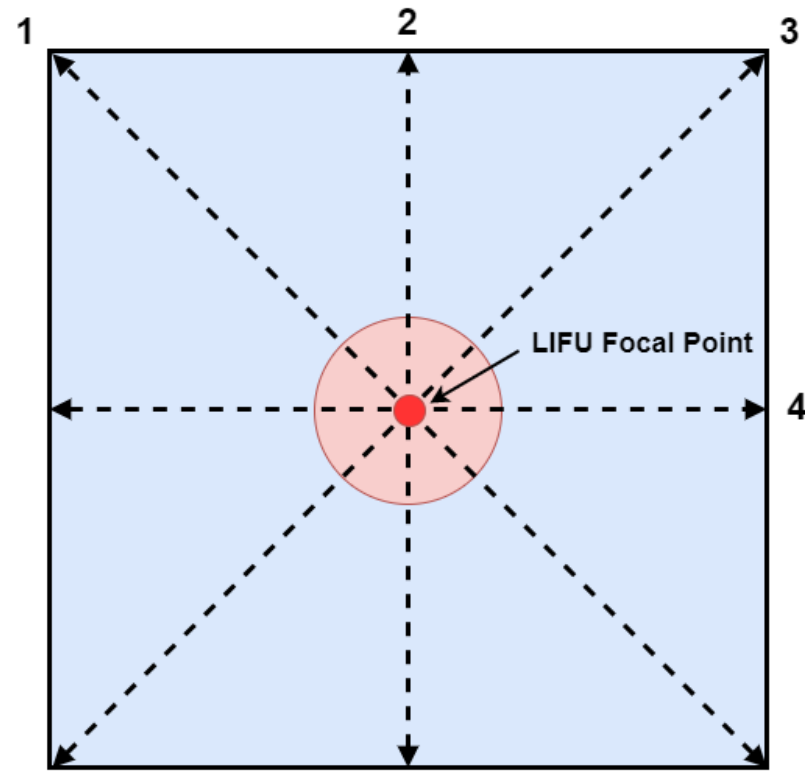

Figure 2.14: Lateral line profile calculation method for experimental 2D temperature maps. Here four line profiles are averaged at different angles through the LIFU transducer focal point. The average lateral temperature profile was then compared to the symmetric COMSOL lateral temperature profile. 


\section{$3 \mid$ Results and Discussion}

\subsection{Development and Optimization of The NLFC Method In Nonlinear B-Mode and BE Imaging}

\subsubsection{Determining NLFC Input Frequencies}

Shown in Figure 3.1 is the calculated SSNR for $B_{\text {Single }}$ images produced for each transmit frequency in the range of 3 to $12.5 \mathrm{MHz}$. All plotted SSNR values were averaged over 3 trials with error bars calculated as the standard error in SSNR at each transmit frequency [90, 91]. Here a peak in SSNR was evident between 4.1 and $7.1 \mathrm{MHz}$, with 12 discrete points within the peak. A symmetric histogram of $B_{N L F C}$ image SSNR produced for every possible frequency pair of these 12 frequencies is depicted in Figure 3.2. From Figure 3.2 it was concluded that by compounding 4.17 and $5.43 \mathrm{MHz}$ as frequency 1 and frequency 2 respectively in the NLFC method, SSNR of the produced $B_{N L F C}$ image was maximized. The same measurements carried out for $B E_{\text {Single }}$ and $B E_{N L F C}$ SSNR are shown in Figures 3.3 and 3.4 respectively. Here a similar trend in $B E_{\text {Single }}$ was seen, with a peak between 4.1 and $7.1 \mathrm{MHz}$. All possible frequency pairs in the SSNR peak were input into the NLFC method, and $B_{N L F C}$ SSNR was maximized when compounding 4.17 and $5.43 \mathrm{MHz}$. It was concluded that in order to maximize SSNR of both $B_{N L F C}$ and $B E_{N L F C}$ images produced with the NLFC method, 4.17 and $5.43 \mathrm{MHz}$ should be selected as input parameters. 


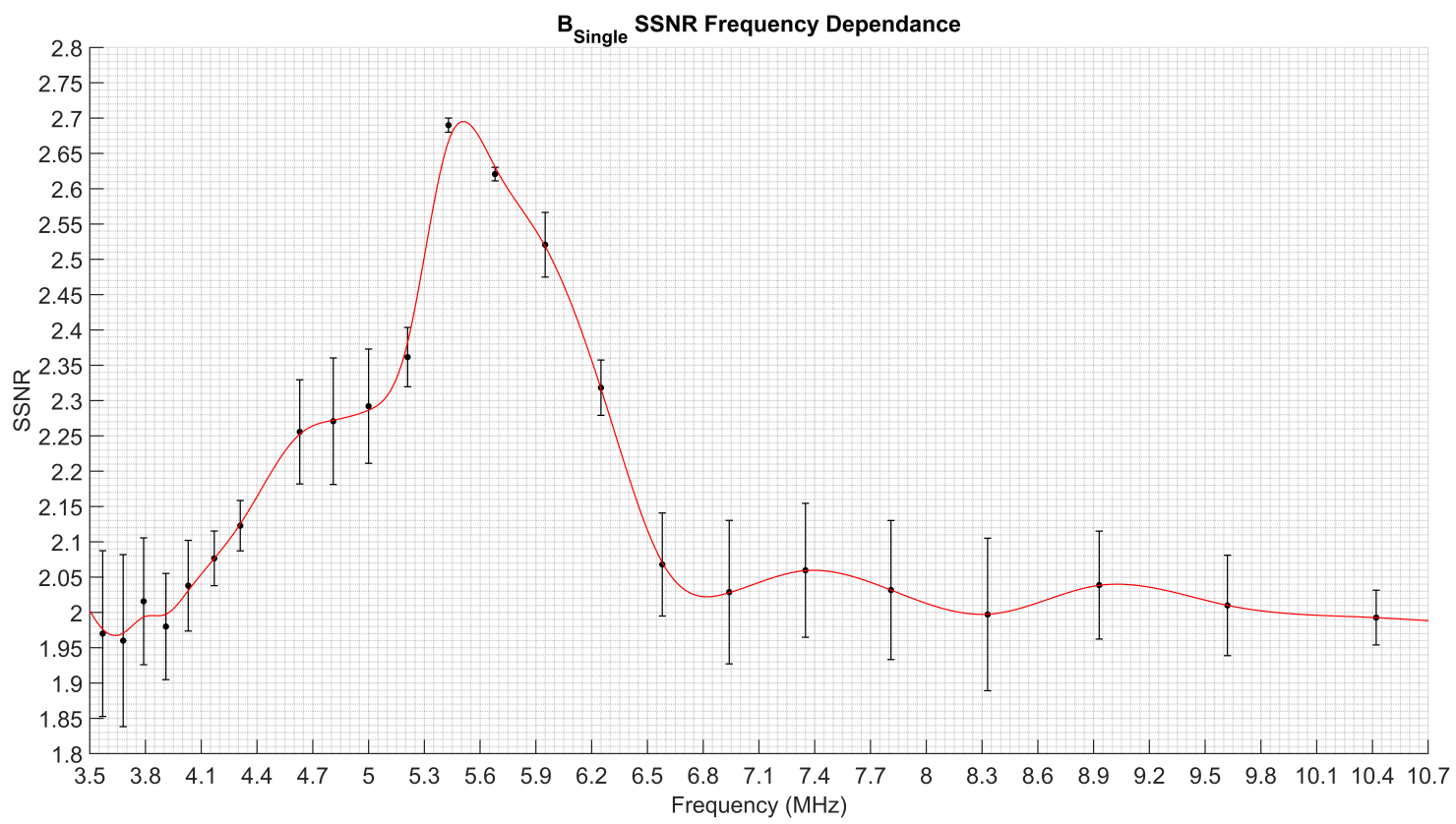

Figure 3.1: $B_{\text {Single }}$ SSNR as a function of transmit frequency. Here a peak is evident between 4.1 $\mathrm{MHz}$ and $7.1 \mathrm{MHz}$, shown in green.

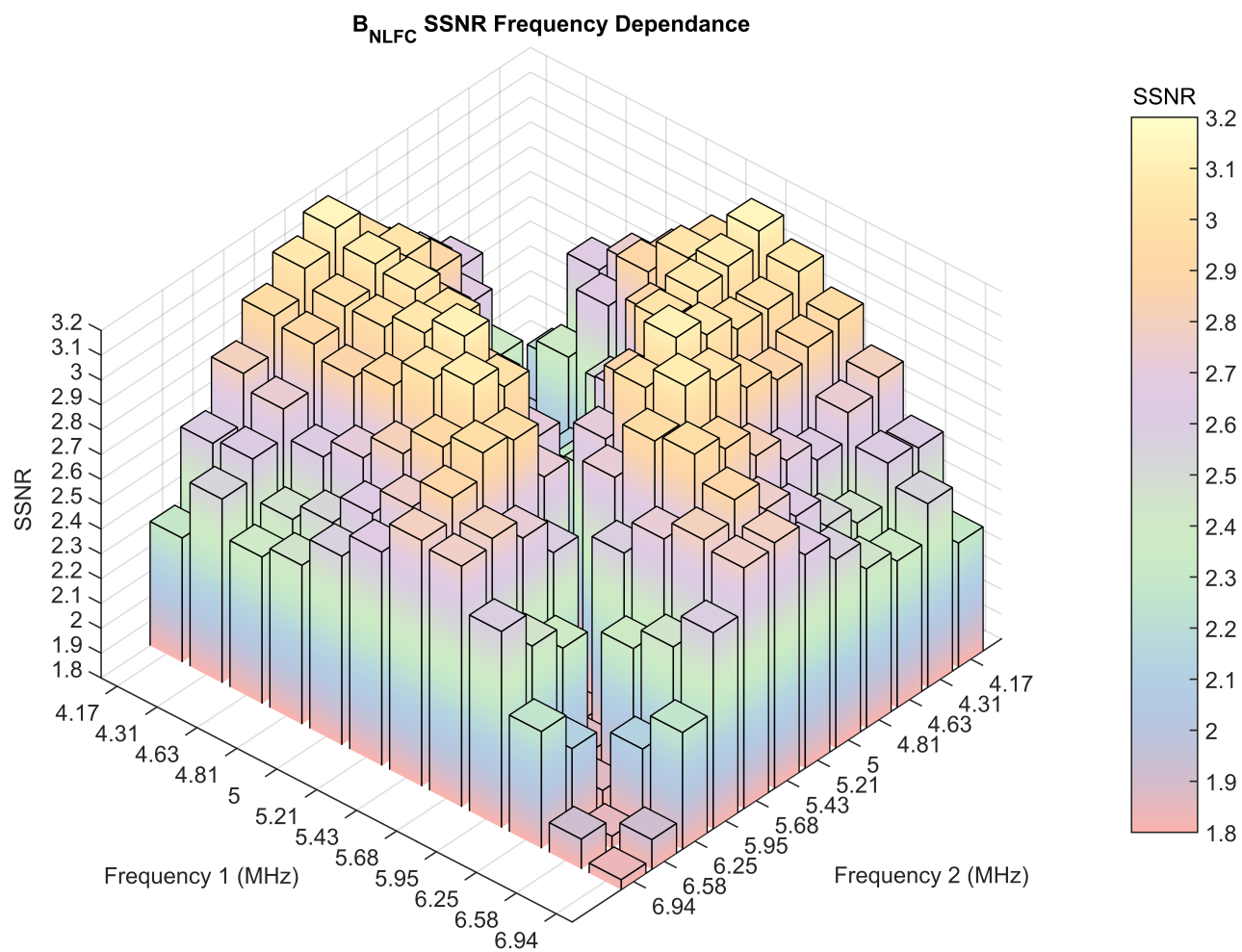

Figure 3.2: All possible frequency pairs in the $B_{\text {Single }}$ SSNR peak were input into the NLFC method, and SSNR of the generated $B_{N L F C}$ image calculated. By compounding $4.17 \mathrm{MHz}$ and $5.43 \mathrm{MHz}$ a maximum SSNR value of 3.16 was achieved. Here the transmit frequencies used for frames 1 and 2 are shown on the $\mathrm{x}$-axis and $\mathrm{y}$-axis respectively. 


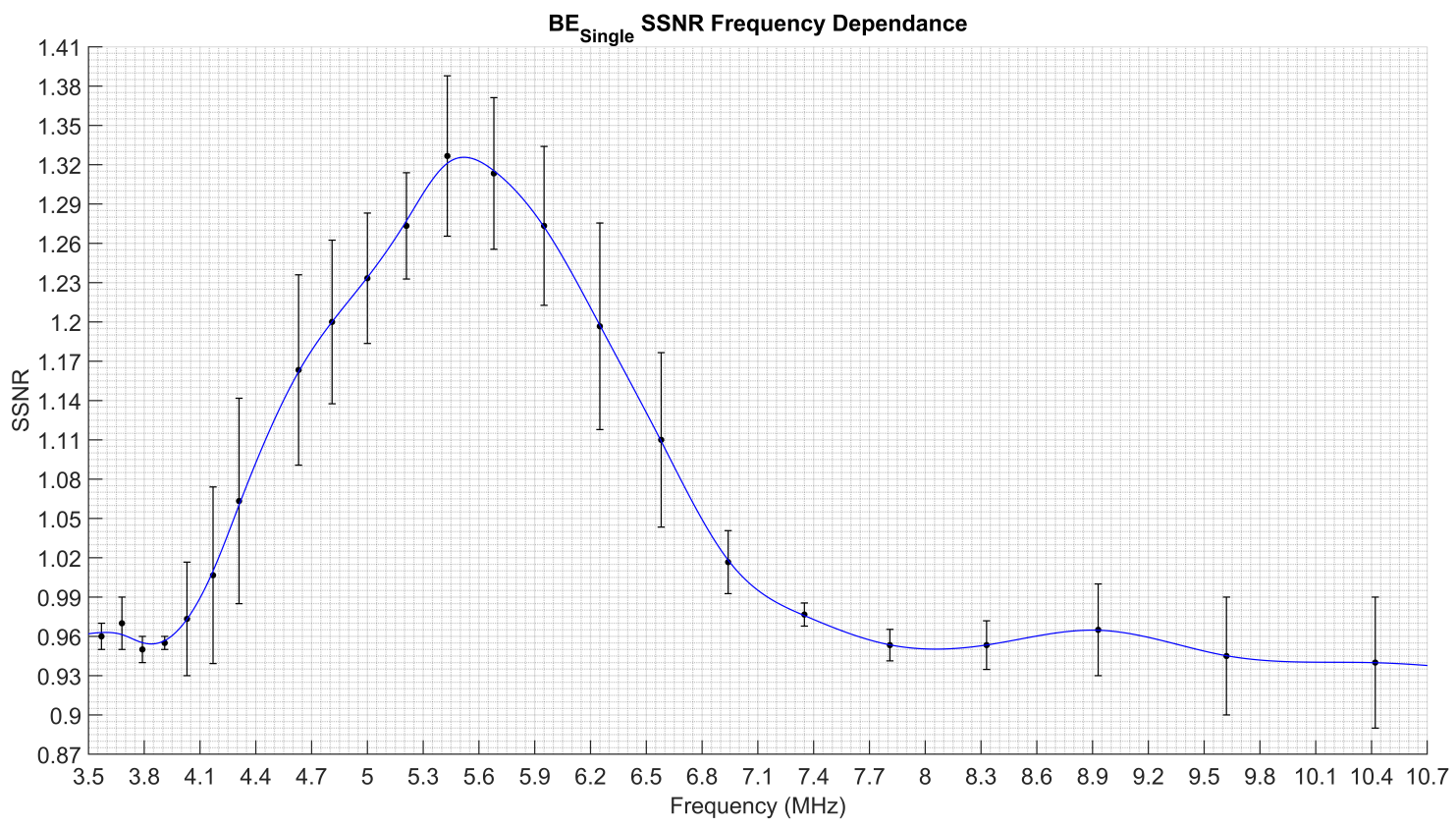

Figure 3.3: $B E_{\text {Single }}$ SSNR as a function of transmit frequency. Here a peak is evident between 4.1 $\mathrm{MHz}$ and $7.1 \mathrm{MHz}$, shown in green.

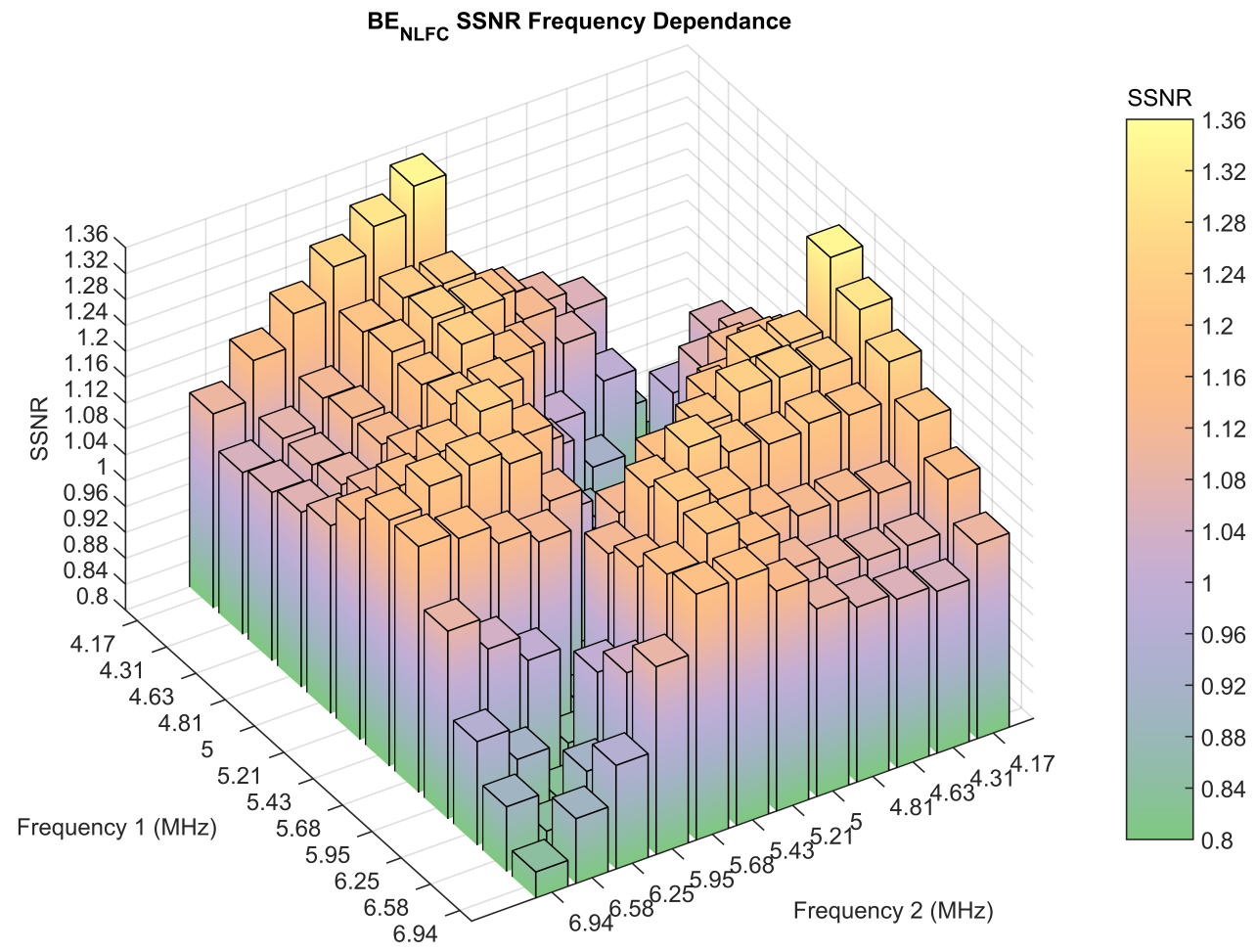

Figure 3.4: All possible frequency pairs in the $B E_{\text {Single }}$ SSNR peak were input into the NLFC method, and SSNR of the generated $B E_{N L F C}$ image calculated. By compounding $4.17 \mathrm{MHz}$ and $5.43 \mathrm{MHz}$ a maximum SSNR value of 3.16 was achieved. Here the transmit frequencies used for frames 1 and 2 are shown on the $\mathrm{x}$-axis and $\mathrm{y}$-axis respectively. 
To ensure that the trend in SSNR of $B_{N L F C}$ was not dependant on the number of cycles, a variety of frequency combinations were also tested with pulses between 2 and 12 cycles. As shown in figure 3.5, by compounding 4.17 and $5.43 \mathrm{MHz}$ with the NLFC method, SSNR was maximized for every tested number of cycles. As a result, 4.17 and $5.43 \mathrm{MHz}$ were selected as frequency 1 and frequency 2 respectively for use with the NLFC method. This frequency pair was then used for all future experiments.

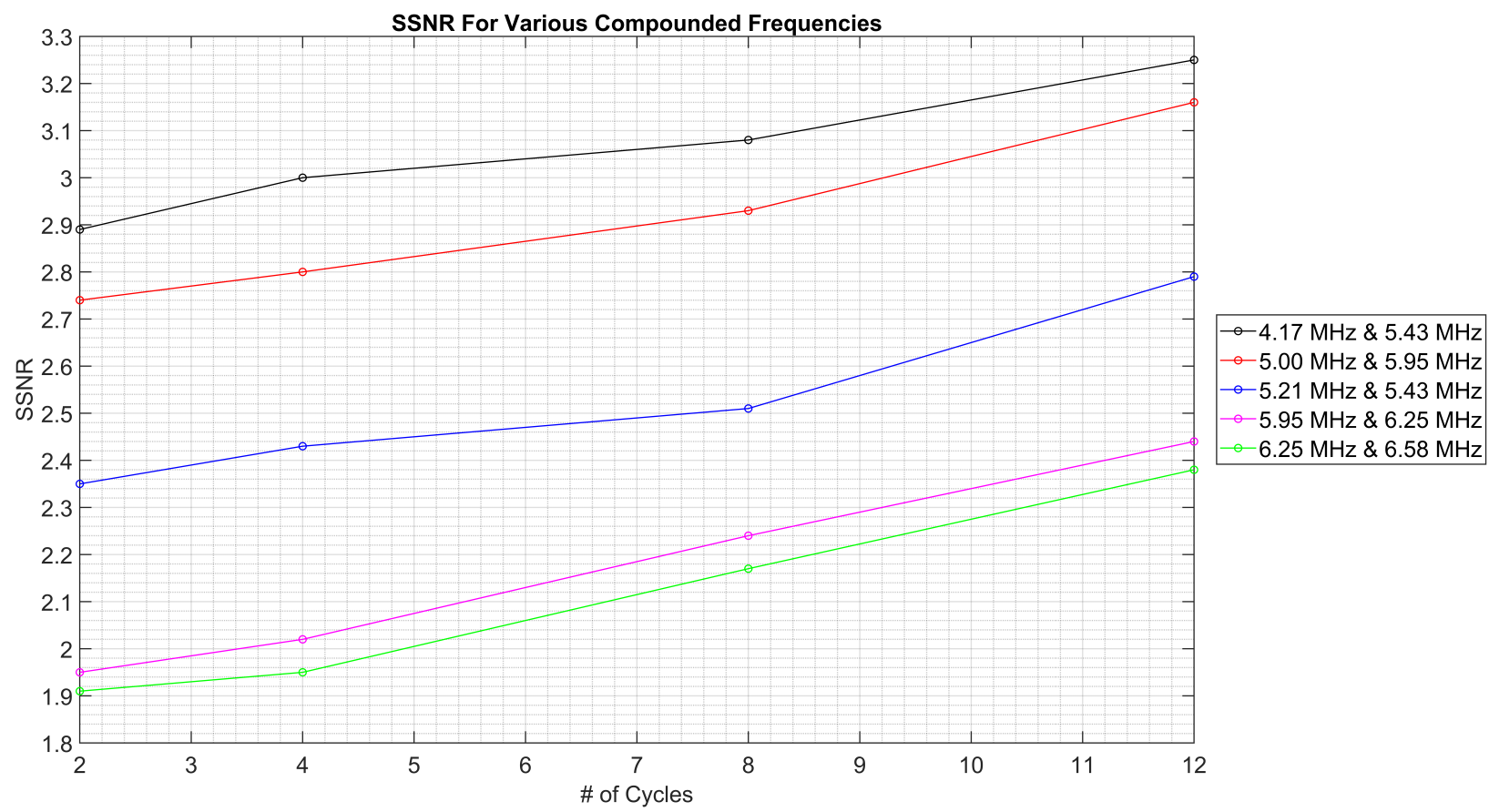

Figure 3.5: Five different combinations of frequencies were inputted into the NLFC method, and B-mode image SSNR calculated for various number of cycles. Regardless of the number of cycles the same trend in SSNR was observed.

\subsubsection{Determining Number of Cycles of Excitation Pulse}

Using a full 3D multi-layer COMSOL based simulation model, a lateral intensity profile at the focal point in porcine tissue was produced for the LIFU transducer, as seen in Figure 3.6. From the FWHM of the lateral intensity profile, a value for the $A R L$ was defined as $1.1 \mathrm{~mm}$. Using Equation 
2.42 a value of $N$ was reported for both $4.17 \mathrm{MHz}$ and $5.43 \mathrm{MHz}$ transmit frequencies as follows:

$$
\begin{aligned}
& N_{1}=\left[\frac{(2)(1.1 \mathrm{~mm})}{\left(1593 \times 10^{3} \mathrm{~mm} / \mathrm{s}\right)}\right]\left(4.17 \times 10^{6} \mathrm{~Hz}\right)=5.76 \\
& N_{2}=\left[\frac{(2)(1.1 \mathrm{~mm})}{\left(1593 \times 10^{3} \mathrm{~mm} / \mathrm{s}\right)}\right]\left(5.43 \times 10^{6} \mathrm{~Hz}\right)=7.50
\end{aligned}
$$

From this it was concluded that the maximum value of $N$ that could be used in the NLFC method, while still maintaining an axial resolution limit of $1.1 \mathrm{~mm}$ was 5 cycles.

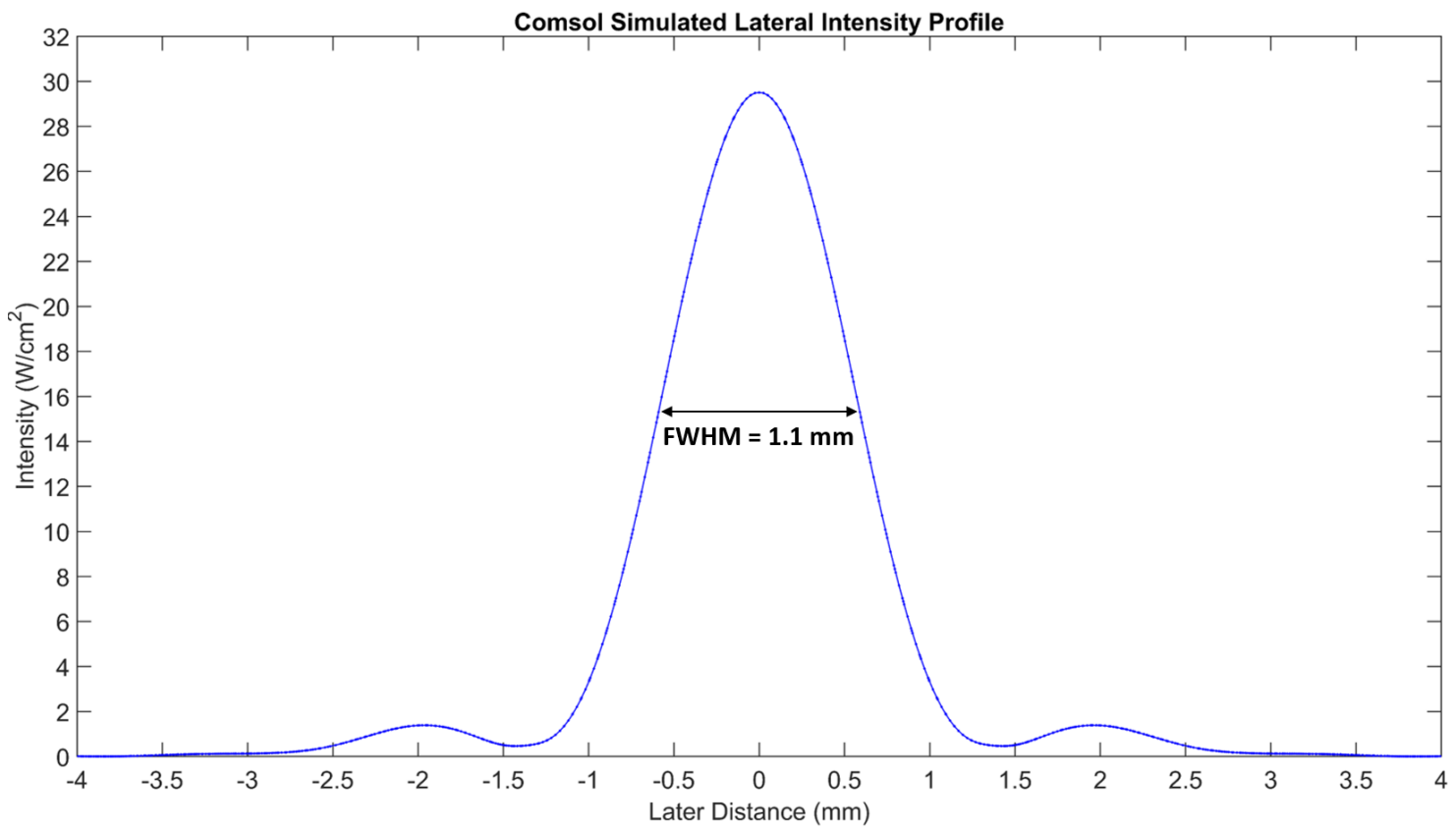

Figure 3.6: COMSOL simulated lateral intensity profile for the LIFU transducer beam at the focal point. The full width half maximum (FWHM) of the intensity profile shows a focal point diameter of $1.1 \mathrm{~mm}$.

\subsubsection{A Comparative Study In B-Mode and BE Image SSNR: NLFC vs NLSF Method}

In order to quantify improvements in SSNR due to the NLFC method, both $B_{N L F C}$ and $B E_{N L F C}$ images were produced with the optimized NLFC input parameters and compared to $B_{\text {Single }}$ and $B E_{\text {Single }}$ images produced with both 4.17 and 5.43 $\mathrm{MHz}$ transmit frequencies. In both methods the number of cycles was kept fixed at 5 cycles to ensure that SSNR was maximized, while still 
maintaining an $A R L$ below $1.1 \mathrm{~mm}$. The calculated SSNR of the $B_{N L F C}, B_{\text {Single }}(4.17 \mathrm{MHz})$ and $B_{\text {Single }}(5.43 \mathrm{MHz})$ images is reported in Table 3.1, along with the percent improvement in SSNR as a result of the NLFC method. The calculated SSNR of the $B E_{N L F C}, B E_{\text {Single }}(4.17 \mathrm{MHz})$ and $B E_{\text {Single }}(5.43 \mathrm{MHz})$ images is reported in Table 3.2, along with the percent improvement in SSNR as a result of the NLFC method. All reported SSNR and percent improvement values are averaged over 6 trials, with uncertainty reported as the standard error.

\begin{tabular}{ccc}
\hline \hline Method & SSNR & NLFC Improvement (\%) \\
\hline NLFC & $2.67 \pm 0.07$ & - \\
NLSF (4.17 MHz) & $1.85 \pm 0.01$ & $44.79 \pm 4.08$ \\
NLSF (5.43 MHz) & $1.88 \pm 0.01$ & $42.28 \pm 4.12$ \\
\hline
\end{tabular}

Table 3.1: Calculated SSNR values for B-mode images generated using the NLFC and NLSF methods. The percent improvement in SSNR by using the NLFC method over the NLSF method is also provided. All values are averaged over 6 trials, with uncertainty reported as the standard error of each value.

\begin{tabular}{ccc}
\hline \hline Method & SSNR & NLFC Improvement (\%) \\
\hline NLFC & $1.34 \pm 0.04$ & - \\
NLSF (4.17 MHz) & $0.96 \pm 0.01$ & $39.65 \pm 4.00$ \\
NLSF (5.43 MHz) & $0.97 \pm 0.01$ & $38.46 \pm 4.72$ \\
\hline
\end{tabular}

Table 3.2: Calculated SSNR values for BE images generated using the NLFC and NLSF methods. The percent improvement in SSNR by using the NLFC method over the NLSF method is also provided. All values are averaged over 6 trials, with uncertainty reported as the standard error of each value.

Here B-mode images generated using the NLSF method had average SSNR values of $1.85 \pm 0.01$ when using a $4.17 \mathrm{MHz}$ transmit frequency, and $1.88 \pm 0.01$ when using a $5.43 \mathrm{MHz}$ transmit frequency. When using the NLFC method, SSNR was raised to an average value of $2.67 \pm 0.07$. This was a $44.79 \pm 4.08 \%$ and $42.28 \pm 4.12 \%$ increase over SSNR of the $4.17 \mathrm{MHz}$ and $5.43 \mathrm{MHz}$ NLSF methods respectively. Here BE images generated using the NLSF method had average SSNR values of $0.96 \pm 0.01$ when using a $4.17 \mathrm{MHz}$ transmit frequency, and $0.97 \pm 0.01$ when using a 5.43 MHz transmit frequency. When using the NLFC method, SSNR was raised to an average value of $1.34 \pm 0.04$. This was a $39.65 \pm 4.00 \%$ and $38.46 \pm 4.72 \%$ increase over SSNR of the 4.17 and 5.43 MHz NLSF methods respectively. From the calculated improvement in SSNR due to the 
NLFC method, it was concluded that the NLFC method is superior to the previously used NLSF method in terms of produced B-mode and BE image SSNR. An example of a NLFC and NLSF B-mode image comparison is provided in Figure 3.7.
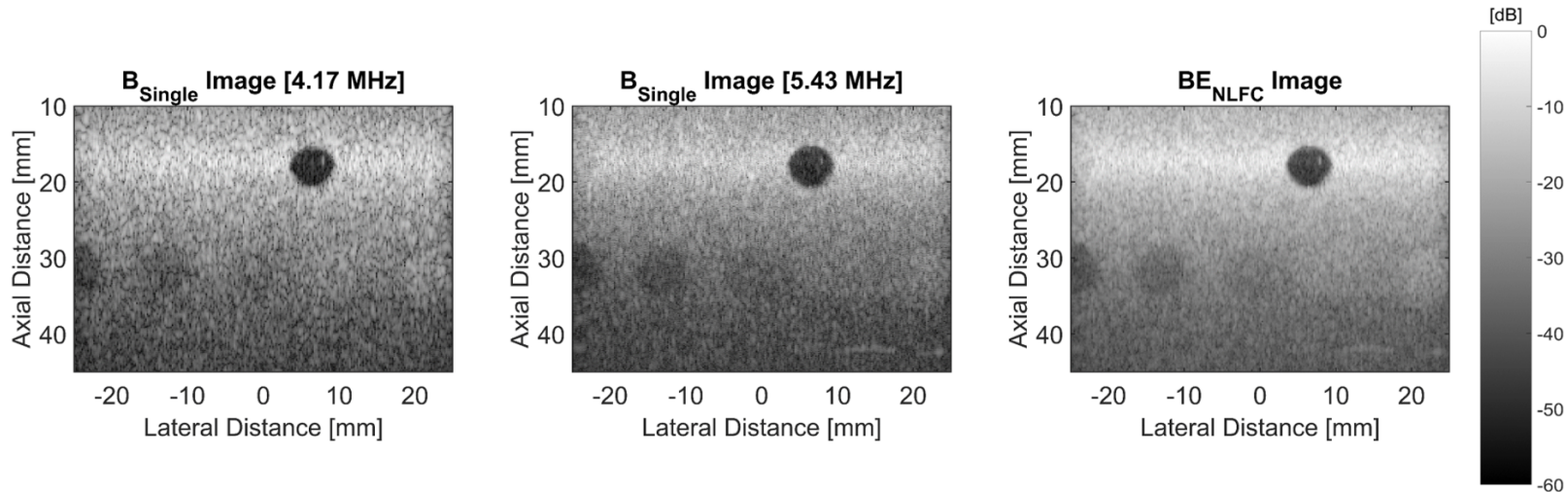

Figure 3.7: A comparison of $B_{N L F C}$ and $B_{\text {Single }}$ B-mode images generated in the TMP. A $B_{\text {Single }}$ image formed using 4.17 MHz (Left) and 5.43 MHz (Middle) transmit frequencies with the NLSF method are shown. When comparing to a $B_{N L F C}$ image formed by compounding 4.17 and 5.43 $\mathrm{MHz}$ (Right) a significant improvement in image quality is seen.

\subsection{Development and Application of The NLFC Method In Non- linear Ultrasound Thermometry}

\subsubsection{LIFU Heating 2D $h$ CBE Maps}

An example of a typical 2D $h \mathrm{CBE}$ map is shown in Figure 3.8 for the NLFC method, and Figures 3.9 and 3.10 for 4.17 and 5.43 MHz NLSF methods respectively. In all three cases a clear hotspot was evident at the LIFU transducer focal point. Both pixel intensity and hotspot radius were shown to grow as heating time is increased, reaching a maximum at six minutes. During the thirty minute cooling phase $h \mathrm{CBE}$ values were expected to return to zero when the initial temperature of $37^{\circ} \mathrm{C}$ was reached. Consistently throughout all trials however, residual nonzero hCBE was observed in the maps even after thirty minutes of cooling. This residual hCBE was deemed an image artifact and attributed to either tissue motion as a result of thermal expansion of the medium, or the generation of a lesion in the tissue sample. Residual artifacts present during the cooling phase 
have been documented in past work with photoacoustic thermometry and are a known issue with some noninvasive thermometry techniques [92]. In all NLFC $h \mathrm{CBE}$ maps produced, the intensity of the residual $h \mathrm{CBE}$ was reduced.
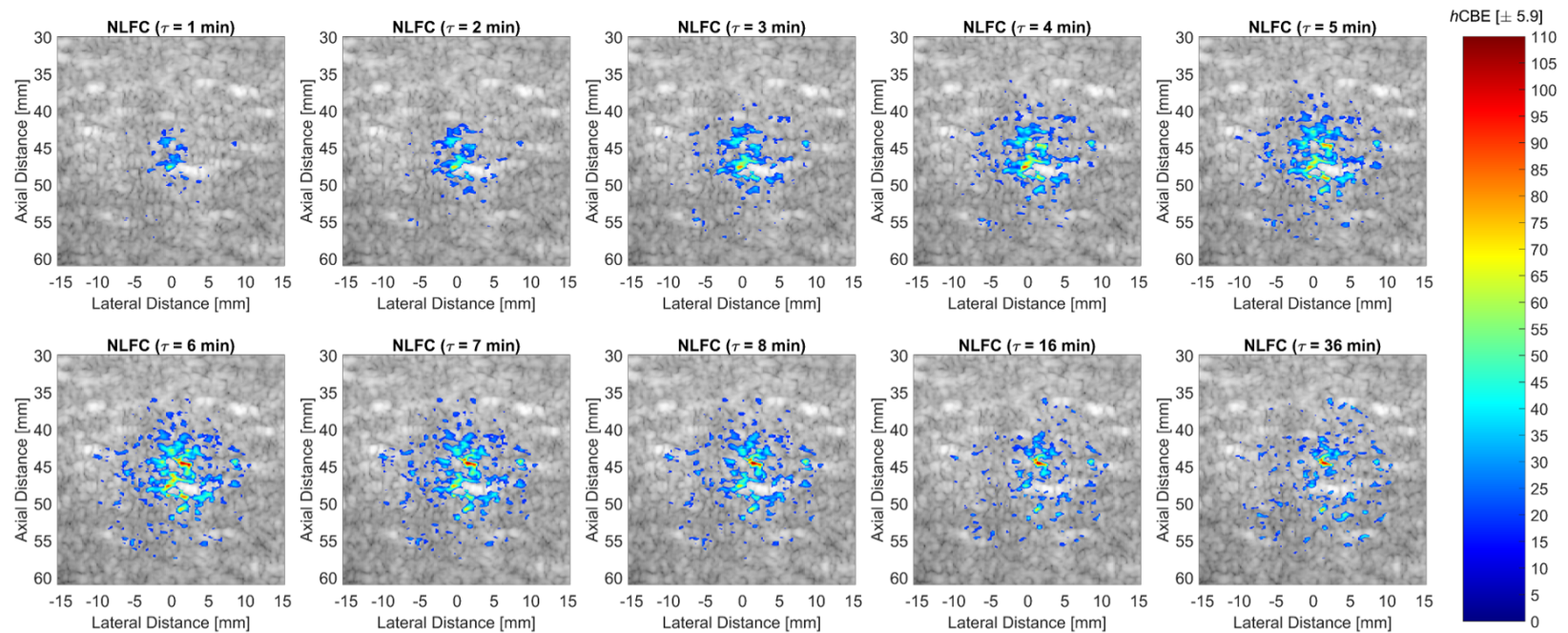

Figure 3.8: A typical $h \mathrm{CBE}$ map for LIFU heating of ex vivo porcine tissue obtained using the NLFC method. The sample was heated for six minutes, then allowed to cool for 30 minutes. Here the $h \mathrm{CBE}$ maps were overlayed on B-mode images of the tissue with a $h \mathrm{CBE}$ plotting threshold set at 15 .
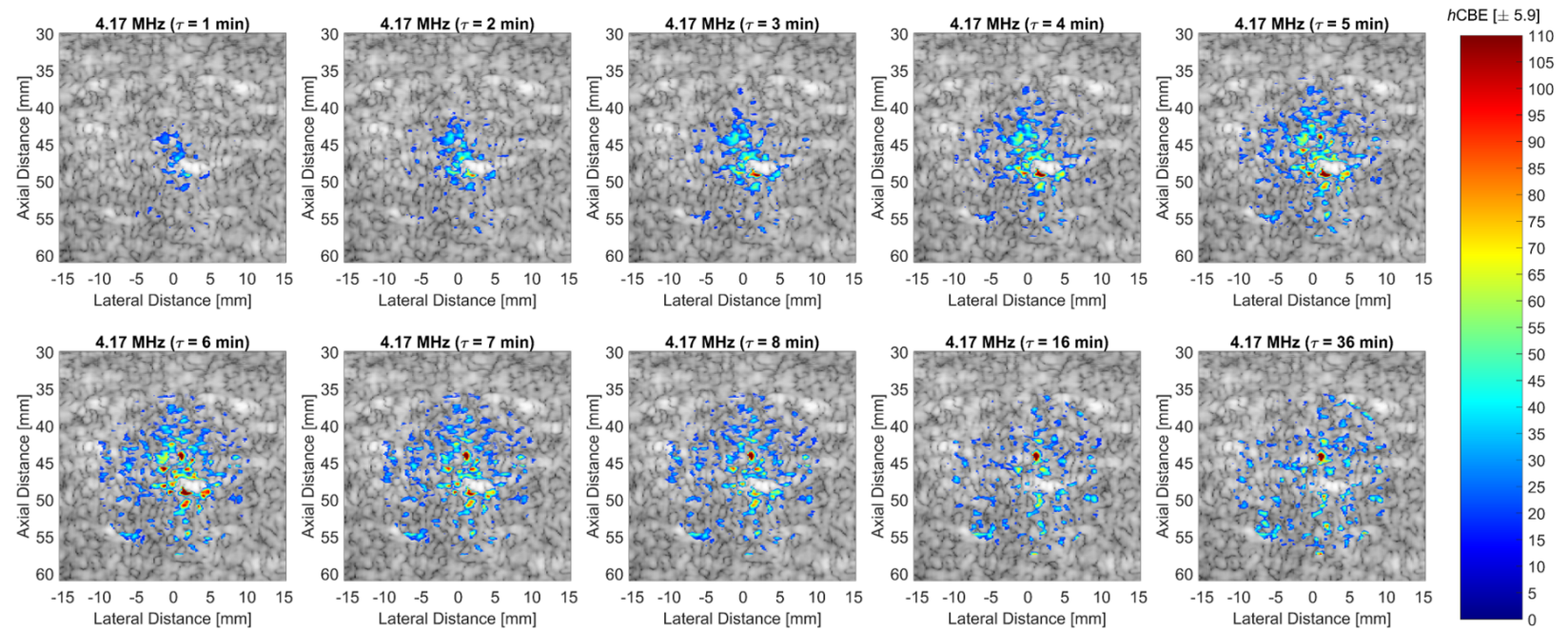

Figure 3.9: A typical $h \mathrm{CBE}$ map for LIFU heating or ex vivo porcine tissue obtained using the NLSF method with a 4.17 MHz transmit frequency. The sample was heated for six minutes, then allowed to cool for 30 minutes. Here the $h \mathrm{CBE}$ maps were overlayed on B-mode images of the tissue with a $h \mathrm{CBE}$ plotting threshold set at 15 . 

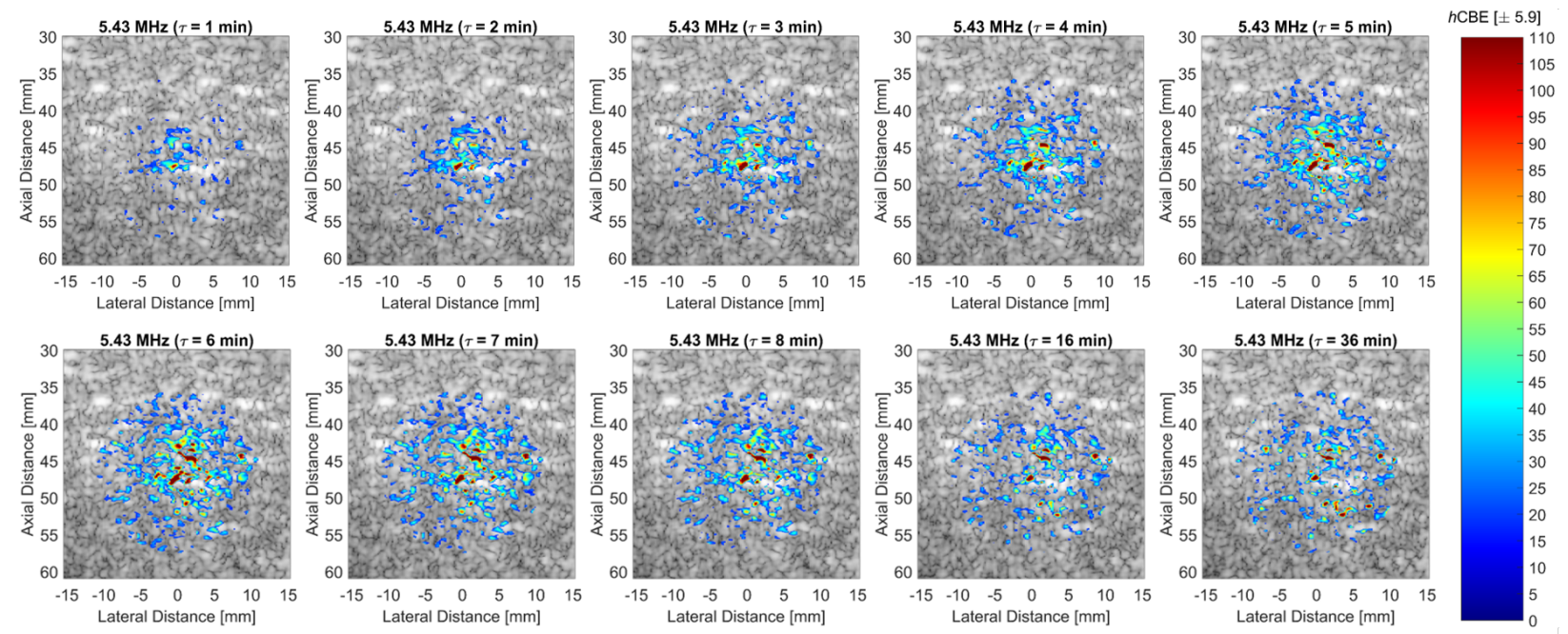

Figure 3.10: A typical $h \mathrm{CBE}$ map for LIFU heating or ex vivo porcine tissue obtained using the NLSF method with a $5.43 \mathrm{MHz}$ transmit frequency. The sample was heated for six minutes, then allowed to cool for 30 minutes. Here the $h \mathrm{CBE}$ maps were overlayed on B-mode images of the tissue with a $h \mathrm{CBE}$ plotting threshold set at 15 .

The NLFC method has integrated motion compensation features in the form of the cumulative sum $h \mathrm{CBE}$ calculation, the 2D motion compensation function, and the gradient filter. By including these motion compensation techniques, motion artifacts in the lateral and axial planes can be reduced, however complex motion in the elevational (tissue thickness) plane could cause false measurements of $h \mathrm{CBE}$. An example of the reduction of motion artifacts using the motion compensation function and gradient filter is provided in Figure 3.11. With the addition of the motion compensation function and gradient filter, a large number of $h \mathrm{CBE}$ artifacts around the edges of the 2D maps can be effectively eliminated, however residual $h \mathrm{CBE}$ was still present during the cooling phase. As shown in Figures 3.6-3.8, a residual $h \mathrm{CBE}$ hotspot of high intensity was evident centered at $(1.5 \mathrm{~mm}, 44.4$ $\mathrm{mm}$ ) throughout the cooling phase. In this trial the highest thermocouple measurement reached at the LIFU transducer focal point at six minutes of heating was $48.5^{\circ} \mathrm{C}$, suggesting the possibility of lesion generation. This could lead to permanent change in tissue properties, which could also contribute to the change in harmonic backscattered energy. 

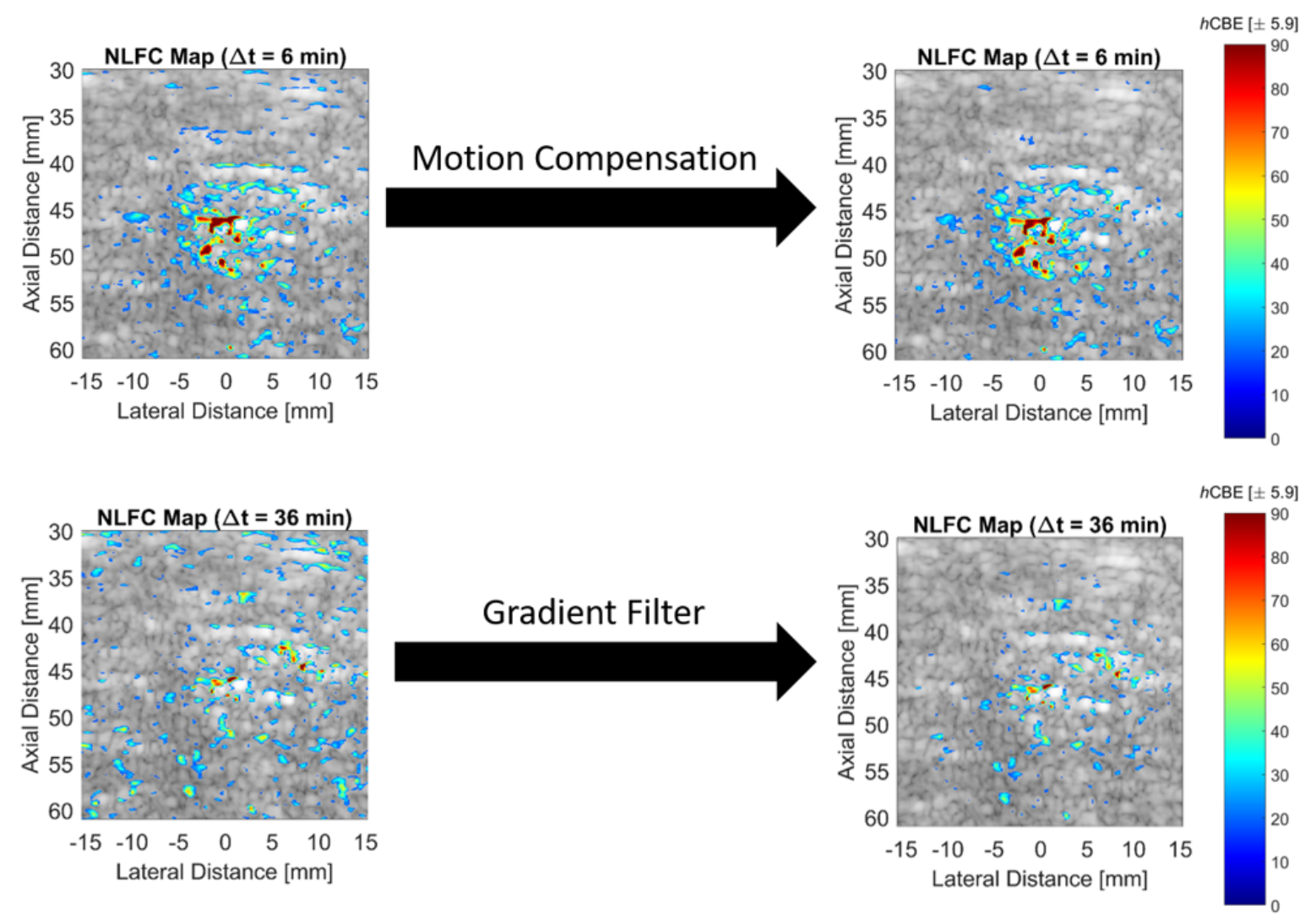

Figure 3.11: (Top) An example of motion artifact reduction in the axial and lateral plane using the cross correlation based motion compensation function. This function can prove advantageous when dealing with measurement-to-measurement motion during the heating phase. (Bottom) An example of artifact reduction at the edges of the 2D map using the gradient filter.

\subsection{2 hCBE-to-Temperature Calibration}

Figure 3.12 depicts the average thermocouple readings and measured $h \mathrm{CBE}$ values for all twelve trials. In order to compare trends in the two measurements, the initial and maximum points were kept fixed. When comparing temperature to $h \mathrm{CBE}$ there was high agreement during the heating phase. Due to the residual $h \mathrm{CBE}$ artifacts present in the cooling phase, the $h \mathrm{CBE}$ curve decreased at a much lower rate than temperature. As a result, only the heating data points were considered in the $h \mathrm{CBE}$-to-temperature calibration curve.

The $h$ CBE-to-temperature curve is shown in Figure 3.13. Here all thermocouple temperature and $h \mathrm{CBE}$ measurements taken during the heating phase are plotted, with a quadratic fit applied to 
the data. The equation of the fit was taken as the $h \mathrm{CBE}$-to-temperature function such that:

$$
T\left[{ }^{\circ} \mathrm{C}\right]=\left[6.34 \times 10^{-4}\right] h C B E^{2}+\left[9.12 \times 10^{-2}\right] h C B E+37.02
$$

Note that here $T$ and $h \mathrm{CBE}$ denote calculated temperature and measured $h \mathrm{CBE}$ respectively. By applying Equation 3.3 to all data points in the NLFC and NLSF hCBE maps, 2D temperature maps for LIFU heating of ex vivo tissue were successfully produced. A typical set of temperature maps produced using both the NLFC method and previous NLSF methods are provided in Figures 3.14-3.16. As seen in the $h \mathrm{CBE}$ maps, there are residual non zero signal artifacts during the cooling phase, but by implementing the NLFC method these artifacts are reduced.

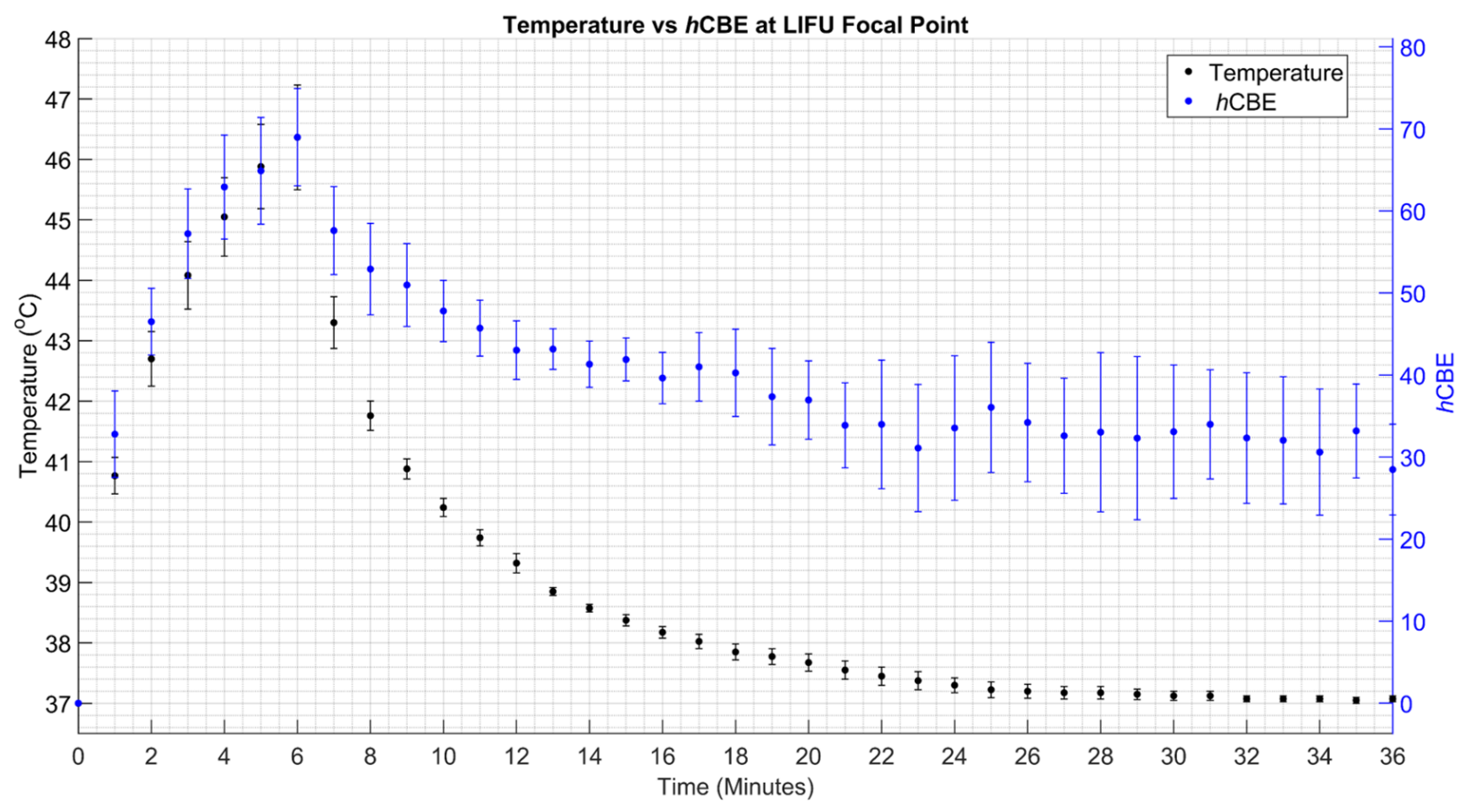

Figure 3.12: Temperature and $h \mathrm{CBE}$ measurements used in the $h \mathrm{CBE}$-to-temperature calibration. Here temperature (shown in black) was determined from a thermocouple placed at the LIFU transducer focal point, while $h \mathrm{CBE}$ (shown in blue) was reported as the mean intensity in a $1 \mathrm{~mm}$ circular ROI centered at the LIFU transducer focal point. 


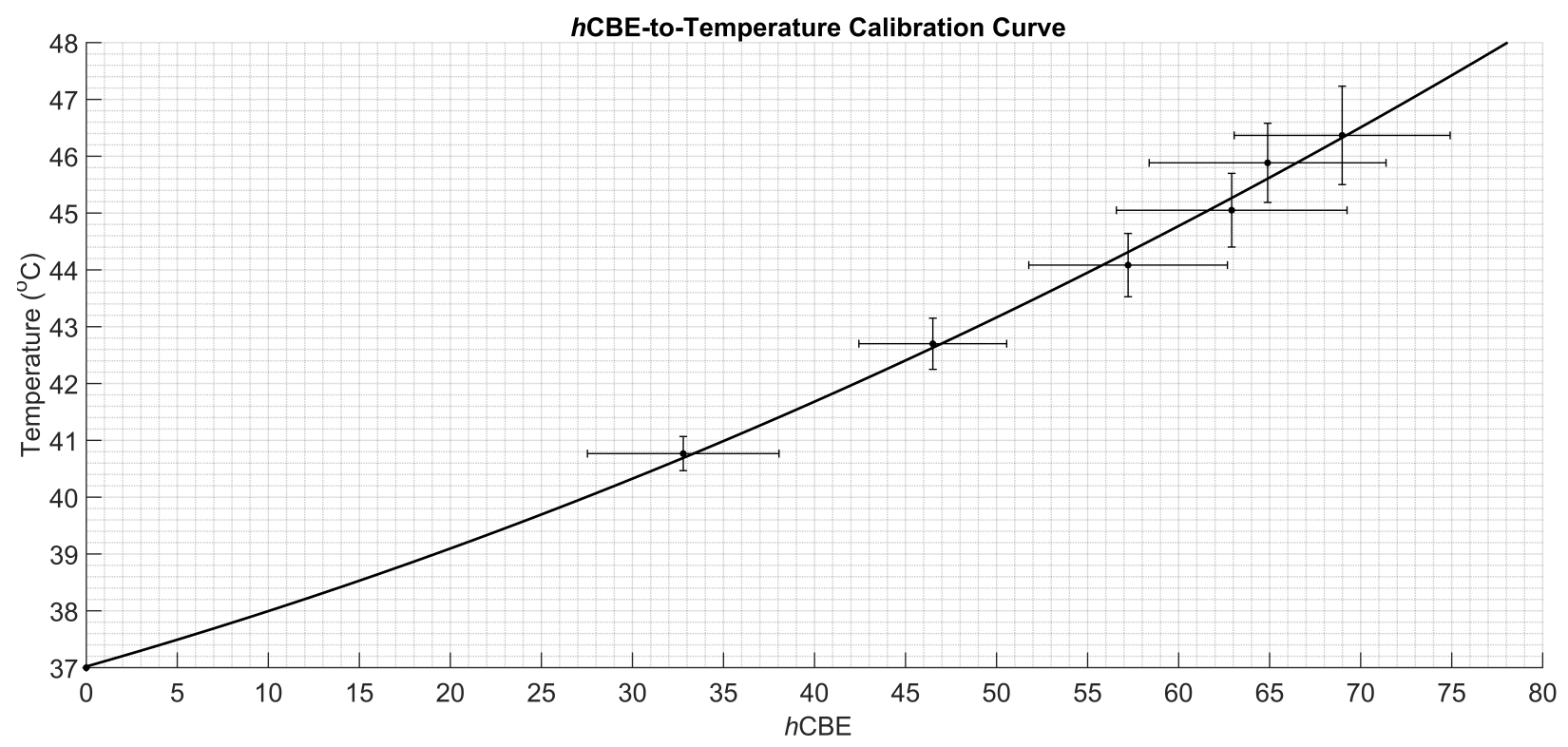

Figure 3.13: $h \mathrm{CBE}$-to-temperature calibration curve. Due to residual $h \mathrm{CBE}$ artifacts appearing during the cooling phase only heating phase data was used for temperature calibration. To produce a calibration function a quadratic fit was applied to the heating phase data.
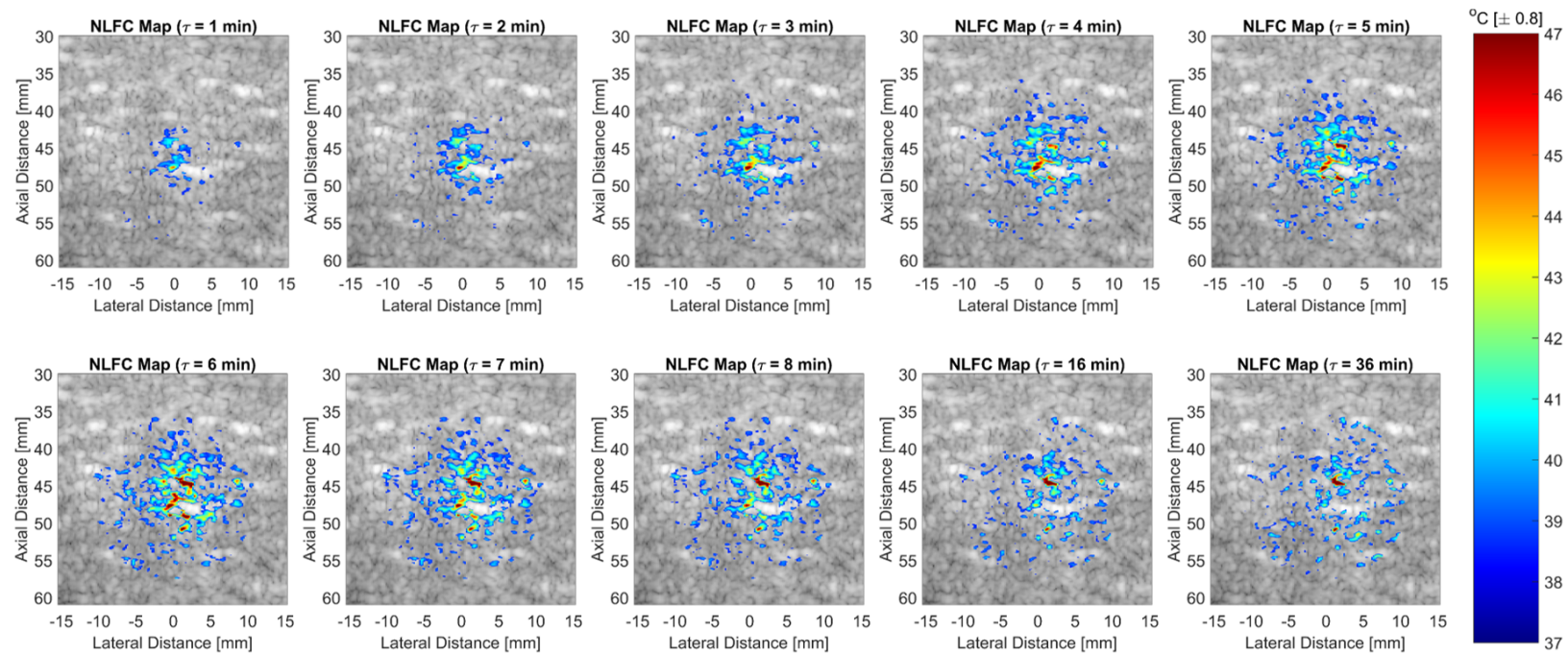

Figure 3.14: A typical 2D temperature map for LIFU heating of ex vivo porcine tissue obtained using the NLFC method. The sample was heated for six minutes, then allowed to cool for 30 minutes. Here the 2D temperature maps were overlayed on B-mode images of the tissue with a $2 \mathrm{D}$ temperature plotting threshold set at $38.5^{\circ} \mathrm{C}(15 \%$ of the maximum increase in temperature reached). 

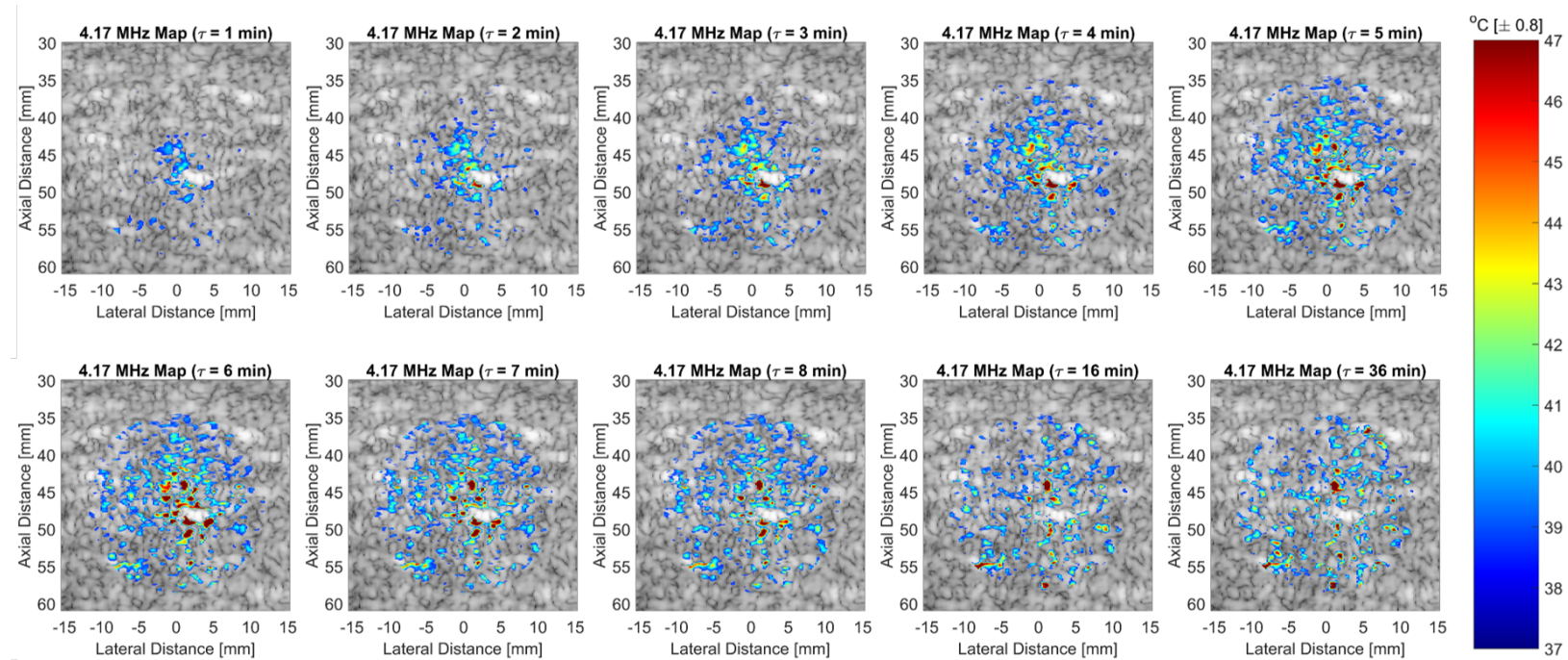

Figure 3.15: A typical 2D temperature map for LIFU heating or ex vivo porcine tissue obtained using the NLSF method with a $4.17 \mathrm{MHz}$ transmit frequency. The sample was heated for six minutes, then allowed to cool for 30 minutes. Here the 2D temperature maps were overlayed on $\mathrm{B}$-mode images of the tissue with a $2 \mathrm{D}$ temperature plotting threshold set at $38.5^{\circ} \mathrm{C}(15 \%$ of the maximum increase in temperature reached).
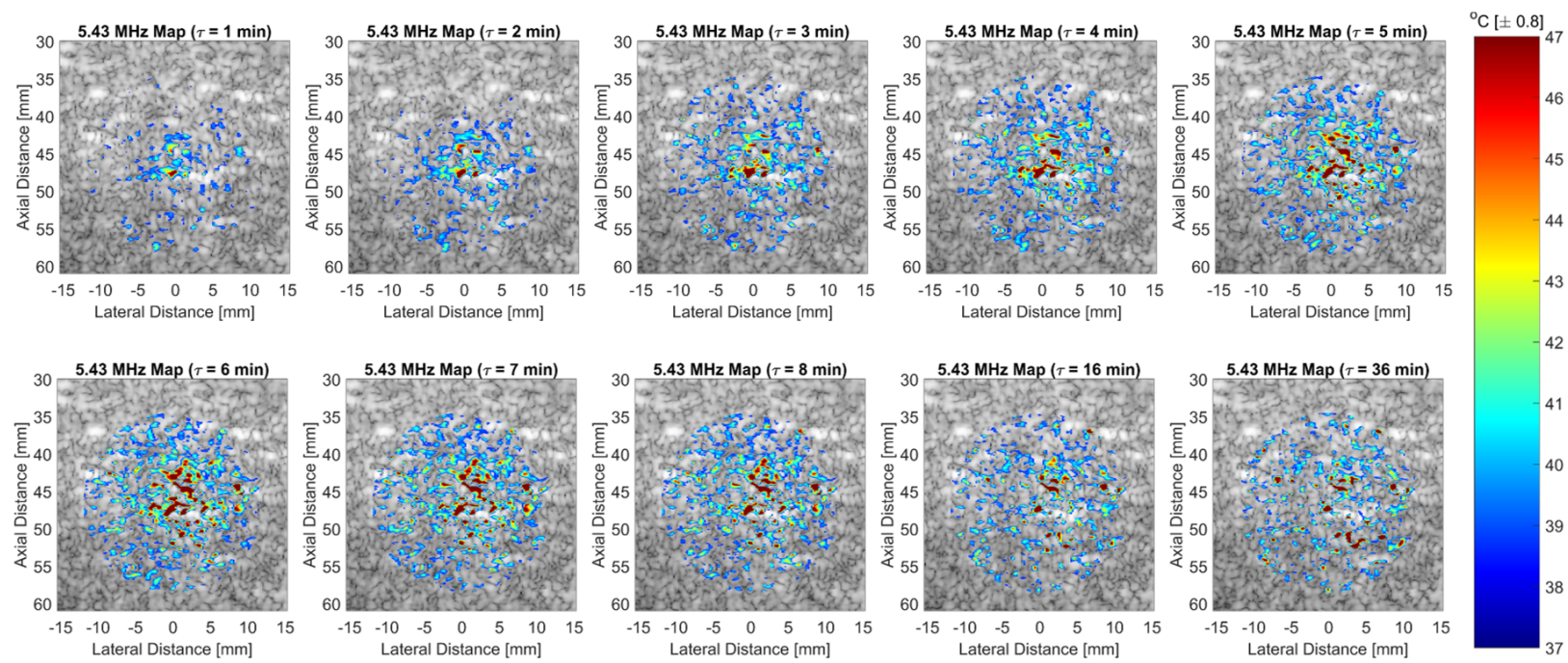

Figure 3.16: A typical 2D temperature map for LIFU heating or ex vivo porcine tissue obtained using the NLSF method with a $5.43 \mathrm{MHz}$ transmit frequency. The sample was heated for six minutes, then allowed to cool for 30 minutes. Here the 2D temperature maps were overlayed on $\mathrm{B}$-mode images of the tissue with a $2 \mathrm{D}$ temperature plotting threshold set at $38.5^{\circ} \mathrm{C}(15 \%$ of the maximum increase in temperature reached).

\subsubsection{A Comparative Study: The NLFC vs NLSF Method}

\section{NLFC SNR Improvements}

In order to quantify the improvement in $2 \mathrm{D}$ (h) $\mathrm{CBE}$ map SNR as a result of the NLFC method, SNR was calculated as a function of heating time for both the NLFC and NLSF $h$ CBE maps. The 
resulting SNR values are provided in Figure 3.17 and Table 3.3. Calculated SNR of heating phase $h$ CBE maps was averaged over all six trials and the NLFC method percent improvement over 4.17 $\mathrm{MHz}$ and 5.43 MHz NLSF maps reported, as seen in Table 3.4.

For every tested 2D $h \mathrm{CBE}$ map, SNR was improved using the NLFC method. Here the average percent improvement in SNR as a result of the NLFC method was $64.13 \pm 4.16 \%$ and $38.72 \pm 2.97 \%$ when compared to the NLSF 4.17 MHz and 5.43 MHz methods respectively. This suggests that by using the NLFC method $h$ CBE maps SNR can be improved significantly over the previous NLSF method.

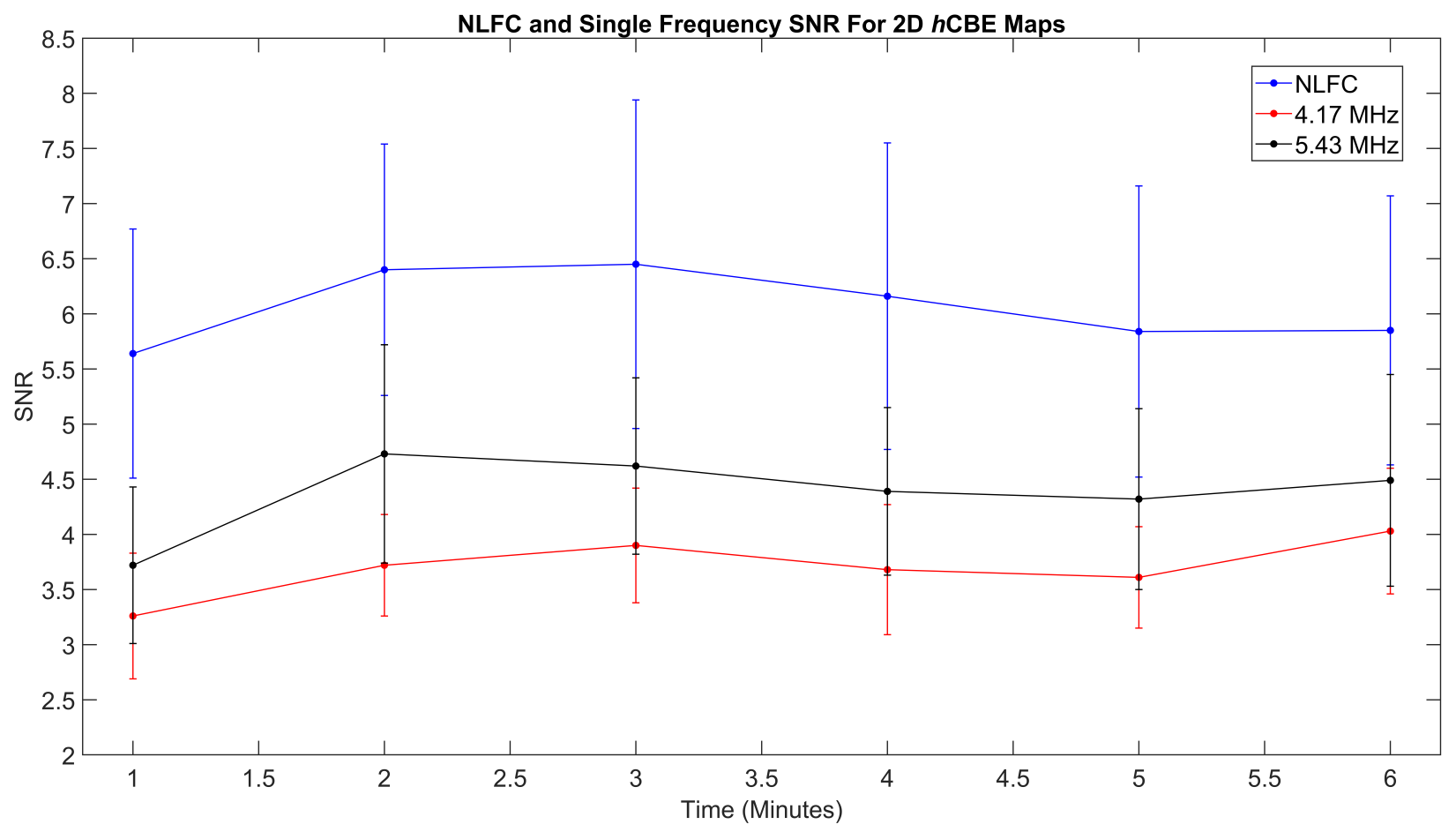

Figure 3.17: Measured SNR of 2D $h \mathrm{CBE}$ maps generated using both the NLFC and NLSF methods for LIFU heating of ex vivo porcine samples. All values are averaged over 6 trials, with uncertainty reported as the standard error of each value. 


\begin{tabular}{cccc}
\hline \hline Time (Min) & NLFC SNR & 4.17 MHz SNR & 5.43 MHz SNR \\
\hline 1 & $5.64 \pm 1.13$ & $3.26 \pm 0.57$ & $3.72 \pm 0.71$ \\
2 & $6.40 \pm 1.14$ & $3.72 \pm 0.46$ & $4.73 \pm 0.99$ \\
3 & $6.45 \pm 1.49$ & $3.90 \pm 0.52$ & $4.62 \pm 0.80$ \\
4 & $6.16 \pm 1.39$ & $3.68 \pm 0.59$ & $4.39 \pm 0.76$ \\
5 & $5.84 \pm 1.32$ & $3.61 \pm 0.46$ & $4.32 \pm 0.82$ \\
6 & $5.85 \pm 1.22$ & $4.03 \pm 0.57$ & $4.49 \pm 0.96$ \\
\hline
\end{tabular}

Table 3.3: SNR of $B E_{N L F C}$ and $B E_{\text {single }} h \mathrm{CBE}$ maps for LIFU heating of ex vivo porcine tissue. All values are averaged over 6 trials, with uncertainty reported as the standard error of each value.

\begin{tabular}{ccc}
\hline \hline Method & SNR & NLFC Improvement (\%) \\
\hline NLFC & $6.06 \pm 1.28$ & - \\
NLSF (4.17 MHz) & $3.70 \pm 0.53$ & $64.13 \pm 4.16$ \\
NLSF (5.43 MHz) & $4.38 \pm 0.84$ & $38.72 \pm 2.97$ \\
\hline
\end{tabular}

Table 3.4: Calculated average SNR values for B-mode images generated using the NLFC and NLSF methods. The percent improvement in SNR by using the NLFC method over the NLSF method is also provided. All values are averaged over 6 trials with 6 heating phase $h \mathrm{CBE}$ maps in each trial (average SNR of 36 maps), with uncertainty reported as the standard error of each value.

\section{Simulated vs Experimental Temperature Maps}

Using COMSOL the LIFU heating experiment was successfully simulated and 2D temperature maps produced, as seen in Figure 3.18. The COMSOL simulated lateral temperature profiles obtained at the LIFU transducer focal point for all 36 time stamps were then compared with the experimental NLSF and NLFC lateral temperature profiles, and the RMSE calculated. For all six LIFU heating trials the RMSE was calculated for every temperature map and averaged. A comparison of average NLFC and NLSF RMSE compared to the simulation temperature maps is depicted in Figure 3.19. Here the RMSE when comparing the NLFC and theoretical model temperature maps was consistently less than the NLSF RMSE. Over six trials, the NLFC methods average RMSE was improved by $32.01 \pm 1.01 \%$ and $52.97 \pm 1.57 \%$ over the 4.17 and $5.43 \mathrm{MHz}$ NLSF trials respectively. The calculated average RMSE are provided in Table 3.5. From this it was 
concluded that the 2D temperature maps produced using the NLFC method show better agreement than the NLSF method when compared with the theoretical model.
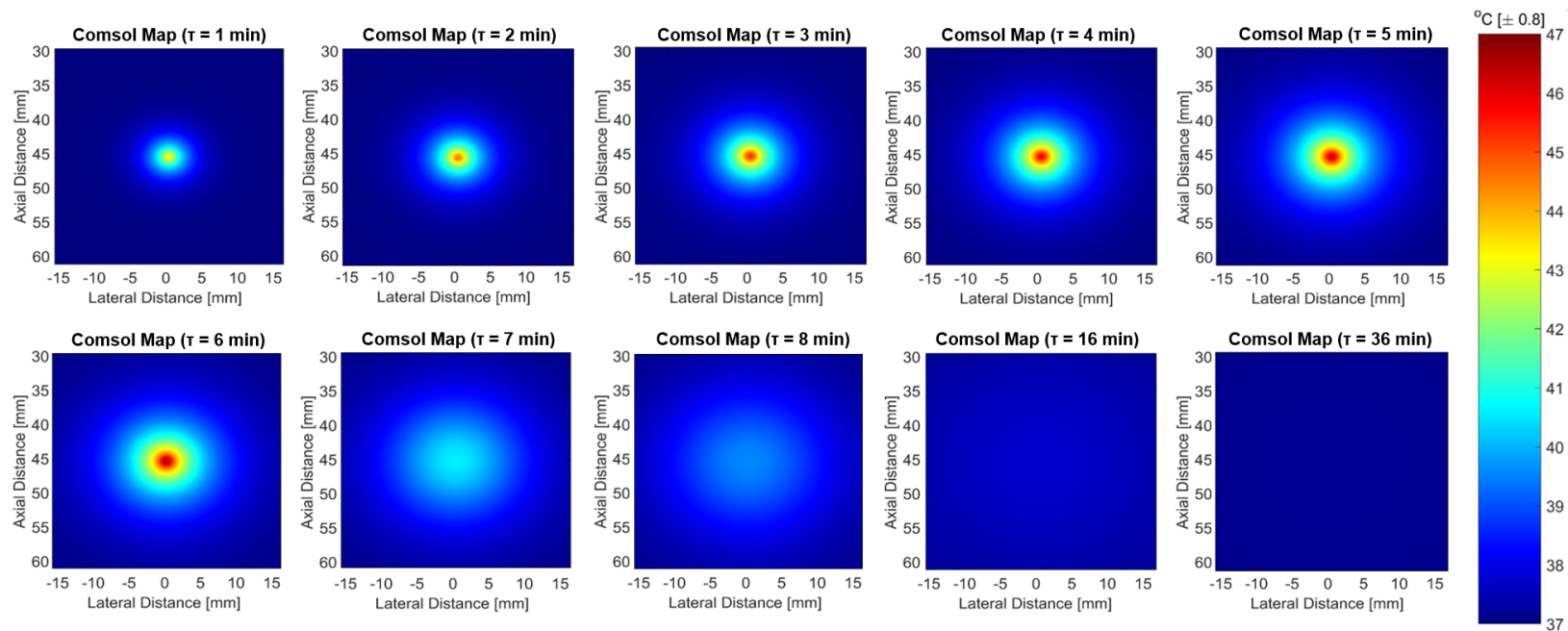

Figure 3.18: A simulated 2D temperature map for LIFU heating of ex vivo porcine tissue obtained using COMSOL with the same medium and exposure parameters as used in the heating experiments.

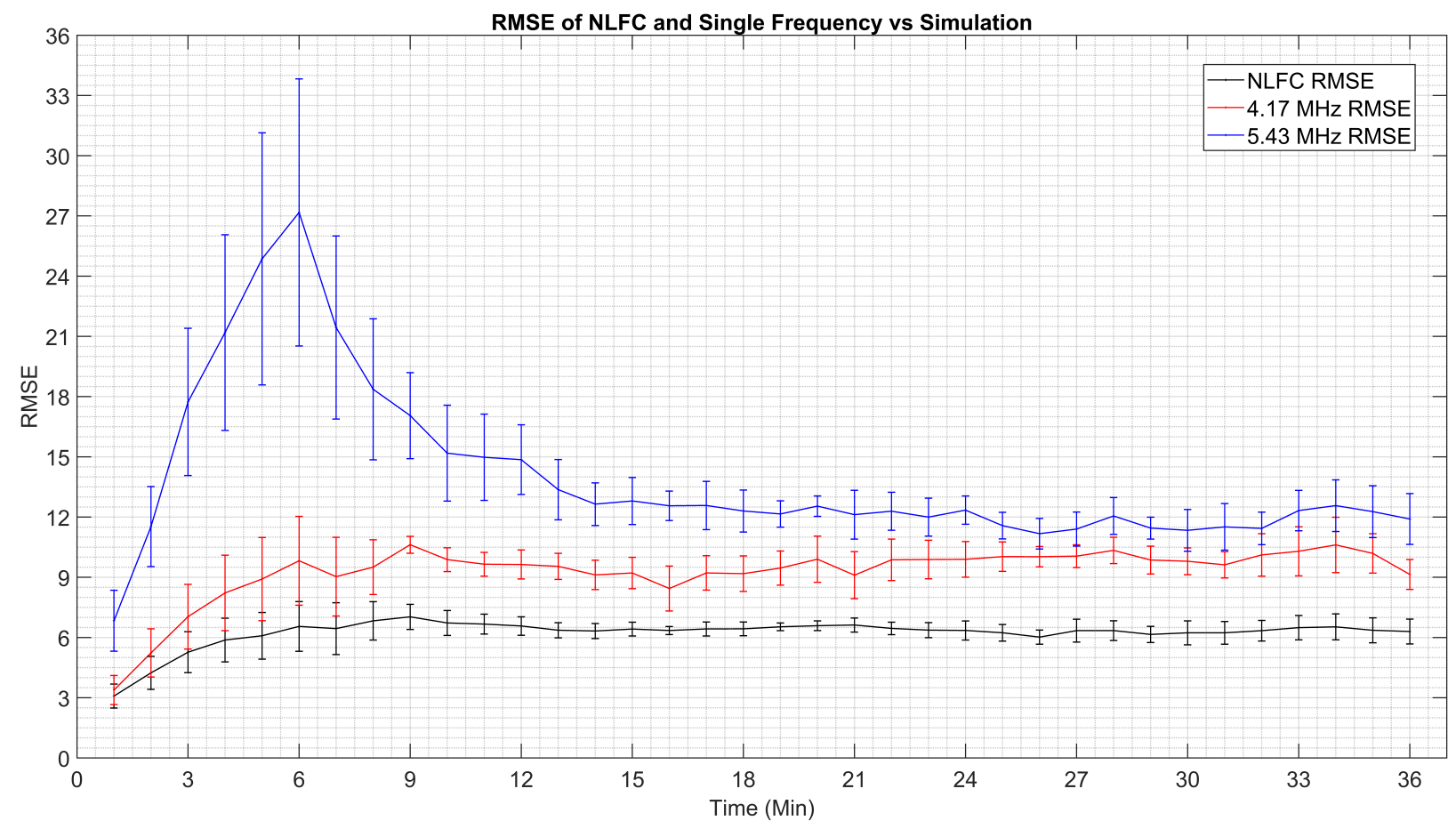

Figure 3.19: RMSE as a function of time for NLFC and NLSF methods compared with COMSOL simulated temperature maps. 


\begin{tabular}{ccc}
\hline \hline Method & RMSE & NLFC Improvement (\%) \\
\hline NLFC & $6.06 \pm 0.56$ & - \\
NLSF (4.17 MHz) & $9.02 \pm 0.98$ & $32.01 \pm 1.01$ \\
NLSF (5.43 MHz) & $13.57 \pm 1.75$ & $52.97 \pm 1.57$ \\
\hline
\end{tabular}

Table 3.5: Calculated RMSE when comparing NLFC and NLSF to simulated COMSOL 2D temperature maps. The percent improvement in RMSE by using the NLFC method over the NLSF method is also provided. All values are averaged over 6 trials, with uncertainty reported as the standard error of each value. 


\section{$4 \mid$ Summary, Conclusions and Future Works}

\subsection{Summary of Work}

The work in this thesis describes a new nonlinear frequency compounding (NLFC) method with applications in B-mode imaging and acoustic harmonic based ultrasound thermometry. In B-mode and BE imaging as well as thermometry applications, the improvements in image quality as a result of the NLFC method were quantified. First, the proposed NLFC method was developed and tested in B-mode and BE imaging of a calibrated tissue mimicking phantom. The transmit frequencies used in the NLFC method were optimized using the tissue mimicking phantom to maximize SSNR of generated B-mode and BE images. The number of cycles used was then determined based on a set axial resolution limit equal to the focal point diameter of the LIFU transducer used in all LIFU heating experiments. Using these optimized parameters an improvement was demonstrated in B-mode and BE image SSNR when compared to the previous NLSF method.

Using a therapeutic LIFU transducer localized heating of ex vivo porcine tissue samples was achieved. Using both the NLFC and NLSF methods 2D maps of $h \mathrm{CBE}$ were produced every minute for a six minute heating period and a thirty minute cooling period. In the generated $h \mathrm{CBE}$ maps a heated region was observed at the LIFU focal point, growing in both intensity and radius as a function of heating time. Over the thirty minute cooling phase the heated region then decreased in both intensity and radius as a function of time. Consistently throughout all trials lingering $h \mathrm{CBE}$ artifacts were observed during the cooling phase. Theoretically when the tissue temperature is returned to $37^{\circ} \mathrm{C}$ all $h \mathrm{CBE}$ values should return to zero, therefore the nonzero $h \mathrm{CBE}$ values seen at the end of the cooling phase were considered as artifacts. In the NLFC method a cumulative sum calculation, 2D motion compensation function, and gradient filter are utilized to reduce lateral 
and axial motion artifacts. However, the thermal expansion leading to motion of the tissue in the elevational plane could contribute to the observed $h \mathrm{CBE}$ artifacts. The potential generation of a thermal lesion in some heating trials could also cause permanent change in tissue properties, producing $h \mathrm{CBE}$ artifacts. From the produced $h \mathrm{CBE}$ maps a $h \mathrm{CBE}$-to-temperature calibration was then performed. Six additional trials of the LIFU heating experiment were performed with an inserted thermocouple at the LIFU transducer focal point, and temperature was measured as a function of time. By plotting measured thermocouple temperature with respect to the average $h \mathrm{CBE}$ at the LIFU transducer focal point, a calibration curve was produced and a quadratic $h \mathrm{CBE}-$ to-temperature calibration function was obtained. 2D maps of temperature were then produced for both the NLFC and NLSF methods and compared with a theoretical COMSOL based model.

Lastly, a comparative study of the NLFC and NLSF method was performed. Here the improvements in $2 \mathrm{D} h \mathrm{CBE}$ map SNR as a result of the new NLFC method was quantified. The average SNR was calculated for all heating phase $h \mathrm{CBE}$ maps over six trials and compared with the previous NLSF method. For all tested $h$ CBE maps, SNR was increased by implementing the NLFC method. The accuracy of the NLFC method in temperature map generation was then tested by comparing NLFC and NLSF temperature maps with a theoretical model in the form of a LIFU heating COMSOL based simulation. A full 3D multi-layer COMSOL based simulation model capable of producing realistic temperature maps was used to this end. For all tested temperature maps the RMSE between the NLFC and simulation maps was much smaller than the NLSF method. This suggests that the NLFC temperature maps are closer to the theoretical model for noninvasive thermometry applications.

\subsection{Conclusions}

Through optimizing NLFC input parameters to maximize SSNR of produced B-mode and BE images, transmit frequencies of 4.17 MHz and 5.43 MHz at a fixed 5-cycle pulse length were selected. Using these input parameters SSNR of B-mode images was raised to an average value of $2.67 \pm 0.07$ compared to $1.85 \pm 0.01$ when using a single $4.17 \mathrm{MHz}$ transmit frequency, and $1.88 \pm 0.01$ when using a single $5.43 \mathrm{MHz}$ transmit frequency. This corresponds to a percent increase of $44.79 \pm 4.08 \%$ and $42.28 \pm 4.12 \%$ over the $4.17 \mathrm{MHz}$ and $5.43 \mathrm{MHz}$ NLSF methods 
respectively. Additionally, BE images generated using the NLSF method had average SSNR values of $0.96 \pm 0.01$ when using a $4.17 \mathrm{MHz}$ transmit frequency, and $0.97 \pm 0.01$ when using a 5.43 $\mathrm{MHz}$ transmit frequency. When using the NLFC method, SSNR was raised to an average value of $1.34 \pm 0.04$. This was a $39.65 \pm 4.00 \%$ and $38.46 \pm 4.72 \%$ increase over SSNR of the 4.17 and 5.43 MHz NLSF methods respectively. From this it was concluded that the NLFC method can generate B-mode and BE images with superior SSNR compared to the previous NLSF method.

The NLFC method was then utilized in acoustic harmonic based ultrasound thermometry for LIFU heating of ex vivo porcine tissue. $2 \mathrm{D}$ maps of $h \mathrm{CBE}$ and temperature were produced and compared with both the NLSF method and COMSOL simulated temperature maps. Due to the lingering $h \mathrm{CBE}$ artifacts observed during the cooling phase only heating phase data was utilized in the $h \mathrm{CBE}$-to-temperature calibration.

When calculating SNR of 2D $h$ CBE maps produced using the NLFC and NLSF methods, the NLFC method was again found to improve image SNR. Here SNR of $2 \mathrm{D} h \mathrm{CBE}$ maps was raised to an average value of $6.06 \pm 1.28$ compared to $3.70 \pm 0.53$ when using a single $4.17 \mathrm{MHz}$ transmit frequency, and $4.38 \pm 0.84$ when using a single $5.43 \mathrm{MHz}$ transmit frequency. This corresponds to a percent increase of $64.13 \pm 4.16 \%$ and $38.72 \pm 2.97 \%$ over the $4.17 \mathrm{MHz}$ and $5.43 \mathrm{MHz}$ NLSF methods respectively. When comparing temperature profiles of the NLFC and NLSF methods with the simulated temperature maps, the RMSE of the NLFC maps was consistently lower for all tested images. Over six trials, the NLFC methods average RMSE was improved by $32.01 \pm 1.01 \%$ and $52.97 \pm 1.57 \%$ over the $4.17 \mathrm{MHz}$ and $5.43 \mathrm{MHz}$ single frequency trials respectively. From this it was concluded that both $h \mathrm{CBE}$ map SNR and the agreement of the $h \mathrm{CBE}$-to-temperature calibration with the theoretical model were improved when using the NLFC method in place of the previously used NLSF method.

\subsection{Future Work}

The work in this thesis can be extended through further testing on $h \mathrm{CBE}$ map generation, to determine the source of cooling phase artifacts. Theoretically, $h \mathrm{CBE}$ should return to zero when the initial baseline temperature is reached. If the source of the lingering $h \mathrm{CBE}$ artifacts observed during 
the cooling phase of all LIFU heating experiments can be determined, the reliability of produced $h \mathrm{CBE}$ maps during the cooling phase could be significantly improved. Once cooling phase artifacts are removed, an additional $h \mathrm{CBE}$-to-temperature calibration could be performed using the cooling phase data.

In order for the NLFC technique to be viable in a clinical setting, its acquisition and processing time must also be reduced for real-time applications. Currently RF data acquisition with the Verasonics scanner can be performed every sixty seconds, however all $h \mathrm{CBE}$ calculations are performed offline. If the generation of $h \mathrm{CBE}$ and temperature maps could be compressed into a single MATLAB code and performed in real time during RF data acquisition, this method would be more clinically viable.

Lastly, additional calibration curves must be generated for other tissue types. The $h \mathrm{CBE}$ to-temperature calibration performed in this work only applies to ex vivo porcine muscle tissue measurements performed with an L12-5 linear array transducer at transmit frequencies of 4.17 $\mathrm{MHz}$, and 5.43 MHz. Before the NLFC method can be applied to thermometry in heterogeneous tissue, additional calibrations must be completed with a wider array of tissues, transducer types, and transmit frequencies. Additionally, an optimal transducer and frequency combination must be determined for clinical use. 


\section{Bibliography}

[1] Aladin Carovac, Fahrudin Smajlovic, and Dzelaludin Junuzovic. "Application of ultrasound in medicine". In: Acta Informatica Medica 19.3 (2011), p. 168.

[2] Wayne Hendrick, David Hykes, and Dale Starchman. Ultrasound Physics and Instrumentation. Elsevier Canada, 2004.

[3] Vincent Chan and Anahi Perlas. "Basics of ultrasound imaging". In: Atlas of ultrasoundguided procedures in interventional pain management. Springer, 2011, pp. 13-19.

[4] Jackie L Whittaker and Maria Stokes. "Ultrasound imaging and muscle function". In: Journal of Orthopaedic \& Sports Physical Therapy 41.8 (2011), pp. 572-580.

[5] Fikri M Abu-Zidan, Ashraf F Hefny, and Peter Corr. "Clinical ultrasound physics". In: Journal of Emergencies, Trauma and Shock 4.4 (2011), p. 501.

[6] Peter C Tay et al. "Ultrasound despeckling for contrast enhancement". In: IEEE Transactions on Image Processing 19.7 (2010), pp. 1847-1860.

[7] Jihoon Park et al. "Speckle reduction techniques in medical ultrasound imaging". In: Biomedical Engineering Letters 4.1 (2014), pp. 32-40.

[8] Joshua S Ullom, Michael L Oelze, and Jose R Sanchez. "Speckle reduction for ultrasonic imaging using frequency compounding and despeckling filters along with coded excitation and pulse compression". In: Advances in Acoustics and Vibration 2012 (2012).

[9] Wei Guo, Yuanyuan Wang, and Jinhua Yu. "Ultrasound harmonic imaging with reducing speckle noise by an interlaced iterative frequency compounding approach". In: 2015 8th International Conference on Biomedical Engineering and Informatics (BMEI). IEEE. 2015, pp. 34-39. 
[10] Muyinatu A Lediju et al. "Quantitative assessment of the magnitude, impact and spatial extent of ultrasonic clutter”. In: Ultrasonic imaging 30.3 (2008), pp. 151-168.

[11] Y Paul et al. "Side lobes and grating lobes artifacts in ultrasound imaging". In: Veterinary radiology \& ultrasound 38.5 (1997), pp. 387-393.

[12] Sonia H Contreras Ortiz, Tsuicheng Chiu, and Martin D Fox. "Ultrasound image enhancement: A review”. In: Biomedical Signal Processing and Control 7.5 (2012), pp. 419-428.

[13] Paul A Magnin, Olaf T von Ramm, and Frederick L Thurstone. "Frequency compounding for speckle contrast reduction in phased array images". In: Ultrasonic imaging 4.3 (1982), pp. 267-281.

[14] Changhan Yoon et al. "Frequency equalized compounding for effective speckle reduction in medical ultrasound imaging”. In: Biomedical Signal Processing and Control 8.6 (2013), pp. 876-887.

[15] Guy Verschaffelt et al. "Speckle disturbance limit in laser-based cinema projection systems". In: Scientific reports 5 (2015), p. 14105.

[16] Vera Behar, Dan Adam, and Zvi Friedman. "A new method of spatial compounding imaging". In: Ultrasonics 41.5 (2003), pp. 377-384.

[17] Ricardo G Dantas and Eduardo T Costa. "Ultrasound speckle reduction using modified Gabor filters". In: IEEE transactions on ultrasonics, ferroelectrics, and frequency control 54.3 (2007), pp. 530-538.

[18] Benoit Mesurolle et al. "Tissue harmonic imaging, frequency compound imaging, and conventional imaging: use and benefit in breast sonography". In: Journal of ultrasound in medicine 26.8 (2007), pp. 1041-1051.

[19] Jose R Sanchez and Michael L Oelze. "An ultrasonic imaging speckle-suppression and contrast-enhancement technique by means of frequency compounding and coded excitation". In: IEEE transactions on ultrasonics, ferroelectrics, and frequency control 56.7 (2009), pp. $1327-1339$.

[20] Jin Ho Chang et al. "Frequency compounded imaging with a high-frequency dual element transducer”. In: Ultrasonics 50.4-5 (2010), pp. 453-457. 
[21] Richard SC Cobbold. Foundations of biomedical ultrasound. Oxford university press, 2006.

[22] F Tranquart et al. "Clinical use of ultrasound tissue harmonic imaging". In: Ultrasound in medicine \& biology 25.6 (1999), pp. 889-894.

[23] Arash Anvari, Flemming Forsberg, and Anthony E Samir. "A primer on the physical principles of tissue harmonic imaging”. In: Radiographics 35.7 (2015), pp. 1955-1964.

[24] Borna Maraghechi et al. "Temperature dependence of acoustic harmonics generated by nonlinear ultrasound wave propagation in water at various frequencies". In: The Journal of the Acoustical Society of America 139.5 (2016), pp. 2475-2481.

[25] See-Ying Chiou et al. "Comparing differential tissue harmonic imaging with tissue harmonic and fundamental gray scale imaging of the liver". In: Journal of Ultrasound in Medicine 26.11 (2007), pp. 1557-1563.

[26] GM Matte et al. "A new frequency compounding technique for super harmonic imaging”. In: 2008 IEEE Ultrasonics Symposium. IEEE. 2008, pp. 357-360.

[27] MG Danilouchkine et al. "Single pulse frequency compounding protocol for superharmonic imaging”. In: Physics in Medicine \& Biology 58.14 (2013), p. 4791.

[28] Gerald L DeNardo and Sally J DeNardo. "Update: Turning the heat on cancer". In: Cancer biotherapy \& radiopharmaceuticals 23.6 (2008), pp. 671-680.

[29] Gian F Baronzio and E Dieter Hager. Hyperthermia in cancer treatment: a primer. Springer Science \& Business Media, 2008.

[30] G Baronzio et al. "A brief overview of hyperthermia in cancer treatment”. In: J Integr Oncol 3.1 (2014), pp. 1-10.

[31] Stephen A Sapareto and William C Dewey. "Thermal dose determination in cancer therapy". In: International Journal of Radiation Oncology* Biology* Physics 10.6 (1984), pp. 787-800.

[32] Lifei Zhu et al. "Ultrasound Hyperthermia Technology for Radiosensitization”. In: Ultrasound in medicine \& biology (2019).

[33] Riadh WY Habash et al. "Thermal therapy, part 1: an introduction to thermal therapy". In: Critical Reviews ${ }^{T M}$ in Biomedical Engineering 34.6 (2006). 
[34] Min Kyu Kang, Myung Se Kim, and Jae Hwang Kim. "Clinical outcomes of mild hyperthermia for locally advanced rectal cancer treated with preoperative radiochemotherapy". In: International Journal of Hyperthermia 27.5 (2011), pp. 482-490.

[35] David Robert Grimes and Mike Partridge. "A mechanistic investigation of the oxygen fixation hypothesis and oxygen enhancement ratio". In: Biomedical physics \& engineering express 1.4 (2015), p. 045209.

[36] Jill Van der Zee. "Heating the patient: a promising approach?” In: Annals of oncology 13.8 (2002), pp. 1173-1184.

[37] Jonathan P May and Shyh-Dar Li. "Hyperthermia-induced drug targeting". In: Expert opinion on drug delivery 10.4 (2013), pp. 511-527.

[38] Miguel N Centelles et al. "Image-guided thermosensitive liposomes for focused ultrasound drug delivery: Using NIRF-labelled lipids and topotecan to visualise the effects of hyperthermia in tumours". In: Journal of Controlled Release 280 (2018), pp. 87-98.

[39] M Thanou and W Gedroyc. "MRI-guided focused ultrasound as a new method of drug delivery". In: Journal of drug delivery 2013 (2013).

[40] Holger Grüll and S Sander Langereis. "Hyperthermia-triggered drug delivery from temperaturesensitive liposomes using MRI-guided high intensity focused ultrasound.” In: Journal of controlled release : official journal of the Controlled Release Society 1612 (2012), pp. 31727.

[41] Meaghan A. O'Reilly and Kullervo Hynynen. "Emerging non-cancer applications of therapeutic ultrasound". In: International Journal of Hyperthermia 31.3 (2015), pp. 310-318.

[42] Thomas P Ryan, Paul F Turner, and Brianne Hamilton. "Interstitial microwave transition from hyperthermia to ablation: historical perspectives and current trends in thermal therapy". In: International Journal of Hyperthermia 26.5 (2010), pp. 415-433.

[43] Jason Chiang, Peng Wang, and Christopher L Brace. "Computational modelling of microwave tumour ablations”. In: International Journal of Hyperthermia 29.4 (2013), pp. 308-317. 
[44] Paul R Stauffer et al. "Conformal microwave array (CMA) applicators for hyperthermia of diffuse chest wall recurrence". In: International Journal of Hyperthermia 26.7 (2010), pp. 686-698.

[45] Annette Klein et al. "Laser thermal therapy of benign skin tumours: Review and update". In: International Journal of Hyperthermia 27.8 (2011), pp. 762-770.

[46] Robert W Ryan et al. "Application of a flexible CO2 laser fiber for neurosurgery: laser-tissue interactions". In: Journal of neurosurgery 112.2 (2010), pp. 434-443.

[47] Jeffrey D Voigt and Gene Barnett. "The value of using a brain laser interstitial thermal therapy (LITT) system in patients presenting with high grade gliomas where maximal safe resection may not be feasible". In: Cost Effectiveness and Resource Allocation 14.1 (2016), p. 6.

[48] R Medvid et al. "Current applications of MRI-guided laser interstitial thermal therapy in the treatment of brain neoplasms and epilepsy: a radiologic and neurosurgical overview". In: American Journal of Neuroradiology 36.11 (2015), pp. 1998-2006.

[49] Hans-Joachim Schwarzmaier et al. "Basic principles of laser induced interstitial thermotherapy in brain tumors". In: Medical Laser Application 17.2 (2002), pp. 147-158.

[50] Xiaohua Huang et al. "Cancer cell imaging and photothermal therapy in the near-infrared region by using gold nanorods". In: Journal of the American Chemical Society 128.6 (2006), pp. $2115-2120$.

[51] Georgy Sergeevich Terentyuk et al. "Laser-induced tissue hyperthermia mediated by gold nanoparticles: toward cancer phototherapy". In: Journal of biomedical optics 14.2 (2009), p. 021016.

[52] Vincenzo Amendola et al. "Surface plasmon resonance in gold nanoparticles: a review". In: Journal of Physics: Condensed Matter 29.20 (2017), p. 203002.

[53] Nardine S Abadeer and Catherine J Murphy. "Recent progress in cancer thermal therapy using gold nanoparticles". In: The Journal of Physical Chemistry C 120.9 (2016), pp. 46914716.

[54] Weibo Cai et al. "Applications of gold nanoparticles in cancer nanotechnology". In: Nanotechnology, science and applications 1 (2008), p. 17. 
[55] Hyunchul Rhim et al. "Essential techniques for successful radio-frequency thermal ablation of malignant hepatic tumors". In: Radiographics 21.suppl_1 (2001), S17-S35.

[56] Riadh WY Habash et al. "Thermal therapy, part 2: hyperthermia techniques". In: Critical Reviews $^{T M}$ in Biomedical Engineering 34.6 (2006).

[57] Douglas L Miller et al. "Overview of therapeutic ultrasound applications and safety considerations". In: Journal of Ultrasound in Medicine 31.4 (2012), pp. 623-634.

[58] Young-sun Kim et al. "High-intensity focused ultrasound therapy: an overview for radiologists”. In: Korean journal of radiology 9.4 (2008), pp. 291-302.

[59] Gail Ter Haar. "Therapeutic applications of ultrasound". In: Progress in biophysics and molecular biology 93.1-3 (2007), pp. 111-129.

[60] Narendra T. Sanghvi Jahan Tavakkoli. "Ultrasound-guided HIFU and Thermal Ablation". In: Therapeutic Ultrasound: Mechanisms to Applications. Ed. by Victor Frenkel. Hauppauge: Nova Science Publishers, 2011. Chap. 6, pp. 137-161.

[61] Gail ter Haar and Constantin Coussios. "High Intensity Focused Ultrasound: Past, present and future”. In: International Journal of Hyperthermia 23.2 (2007), pp. 85-87.

[62] Kullervo Hynynen Chris J Diederich. "Ultrasound technology for hyperthermia”. In: Ultrasound in Medicine \& Biology 25.6 (1999), pp. 871-887.

[63] Matthew A Lewis, Robert M Staruch, and Rajiv Chopra. "Thermometry and ablation monitoring with ultrasound". In: International Journal of Hyperthermia 31.2 (2015), pp. 163181.

[64] I Rivens et al. "Treatment monitoring and thermometry for therapeutic focused ultrasound". In: International journal of hyperthermia 23.2 (2007), pp. 121-139.

[65] Subhashish Dasgupta et al. "Beam localization in HIFU temperature measurements using thermocouples, with application to cooling by large blood vessels". In: Ultrasonics 51.2 (2011), pp. 171-180.

[66] Viola Rieke and Kim Butts Pauly. "MR thermometry”. In: Journal of Magnetic Resonance Imaging: An Official Journal of the International Society for Magnetic Resonance in Medicine 27.2 (2008), pp. 376-390. 
[67] Belykh Evgenii et al. "Laser application in neurosurgery". In: Surgical neurology international 8.274 (2017).

[68] Shintaro Ichikawa et al. "MR-guided focused ultrasound for uterine fibroids: a preliminary study of relationship between the treatment outcomes and factors of mr images including elastography”. In: Magnetic Resonance in Medical Sciences 18.1 (2019), pp. 82-87.

[69] RM Arthur et al. "Non-invasive estimation of hyperthermia temperatures with ultrasound". In: International journal of hyperthermia 21.6 (2005), pp. 589-600.

[70] Christopher Rowland Hill, Jeff C Bamber, and Gail R ter Haar. Physical principles of medical ultrasonics. 2004.

[71] Min Joo Choi et al. "Changes in ultrasonic properties of liver tissue in vitro during heatingcooling cycle concomitant with thermal coagulation". In: Ultrasound in medicine \& biology 37.12 (2011), pp. 2000-2012.

[72] Christakis A Damianou et al. "Dependence of ultrasonic attenuation and absorption in dog soft tissues on temperature and thermal dose". In: The Journal of the Acoustical Society of America 102.1 (1997), pp. 628-634.

[73] Borna Maraghechi, Michael C Kolios, and Jahan Tavakkoli. “Temperature dependence of acoustic harmonics generated by nonlinear ultrasound beam propagation in ex vivo tissue and tissue-mimicking phantoms". In: International Journal of Hyperthermia 31.6 (2015), pp. 666-673.

[74] Claudio Simon, Philip VanBaren, and Emad S Ebbini. "Two-dimensional temperature estimation using diagnostic ultrasound". In: IEEE transactions on ultrasonics, ferroelectrics, and frequency control 45.4 (1998), pp. 1088-1099.

[75] Naomi R Miller, Jeffrey C Bamber, and Paul M Meaney. "Fundamental limitations of noninvasive temperature imaging by means of ultrasound echo strain estimation". In: Ultrasound in medicine \& biology 28.10 (2002), pp. 1319-1333.

[76] Piotr Kielczyński et al. "Thermodynamic method for measuring the B/A nonlinear parameter under high pressure". In: 2013 IEEE International Ultrasonics Symposium (IUS). IEEE. 2013, pp. 1665-1667. 
[77] Francis A Duck. "Nonlinear acoustics in diagnostic ultrasound". In: Ultrasound in medicine \& biology 28.1 (2002), pp. 1-18.

[78] Xiaozhou Liu et al. "Noninvasive estimation of temperature elevations in biological tissues using acoustic nonlinearity parameter imaging”. In: Ultrasound in medicine \& biology 34.3 (2008), pp. 414-424.

[79] Koen WA van Dongen and Martin D Verweij. "A feasibility study for non-invasive thermometry using non-linear ultrasound”. In: International Journal of Hyperthermia 27.6 (2011), pp. 612-624.

[80] R Martin Arthur et al. "In vivo change in ultrasonic backscattered energy with temperature in motion-compensated images". In: International journal of hyperthermia 24.5 (2008), pp. 389-398.

[81] R Martin Arthur et al. "Noninvasive temperature estimation based on the energy of backscattered ultrasound”. In: Medical Physics 30.6 (2003), pp. 1021-1029.

[82] Borna Maraghechi. "Feasibility of Noninvasive Thermometry In Hyperthermia Regime Using Harmonics Generated By Nonlinear Ultrasound Wave Propagation”. PhD thesis. Ryerson University, 2016.

[83] Roger Gent. Applied physics and technology of diagnostic ultrasound. Milner publishing, 1997.

[84] COMSOL Multiphysics ${ }^{\circledR}$ v. 5.4. Application ID: 12659, Focused Ultrasound Induced Heating in Tissue Phantom. 1999. uRL: https: / / www. comsol . com/model/focusedultrasound-induced-heating-in-tissue-phantom-12659.

[85] S Suprijanto et al. "Inter-frame motion correction for MR thermometry". In: International Conference on Medical Image Computing and Computer-Assisted Intervention. Springer. 2005, pp. 580-588.

[86] Francis A Duck. Physical properties of tissues: a comprehensive reference book. Academic press, 2013.

[87] Servet Gulum Sumnu and Serpil Sahin. Advances in deep-fat frying of foods. CRC Press, 2008. 
[88] Alycen Wiacek et al. "Robust Short-Lag Spatial Coherence Imaging of Breast Ultrasound Data: Initial Clinical Results”. In: IEEE transactions on ultrasonics, ferroelectrics, and frequency control 66.3 (2018), pp. 527-540.

[89] Eenas A Omari and Tomy Varghese. "Signal to noise ratio comparisons for ultrasound attenuation slope estimation algorithms". In: Medical physics 41.3 (2014), p. 032902.

[90] George W Brown. "Standard deviation, standard error: Which 'standard' should we use?" In: American Journal of Diseases of Children 136.10 (1982), pp. 937-941.

[91] Leo M Harvill. "Standard error of measurement". In: Educational measurement: Issues and practice 10.2 (1991), pp. 33-41.

[92] Sergey M Nikitin, Tatyana D Khokhlova, and Ivan M Pelivanov. "Temperature dependence of the optoacoustic transformation efficiency in ex vivo tissues for application in monitoring thermal therapies". In: Journal of biomedical optics 17.6 (2012), p. 061214. 